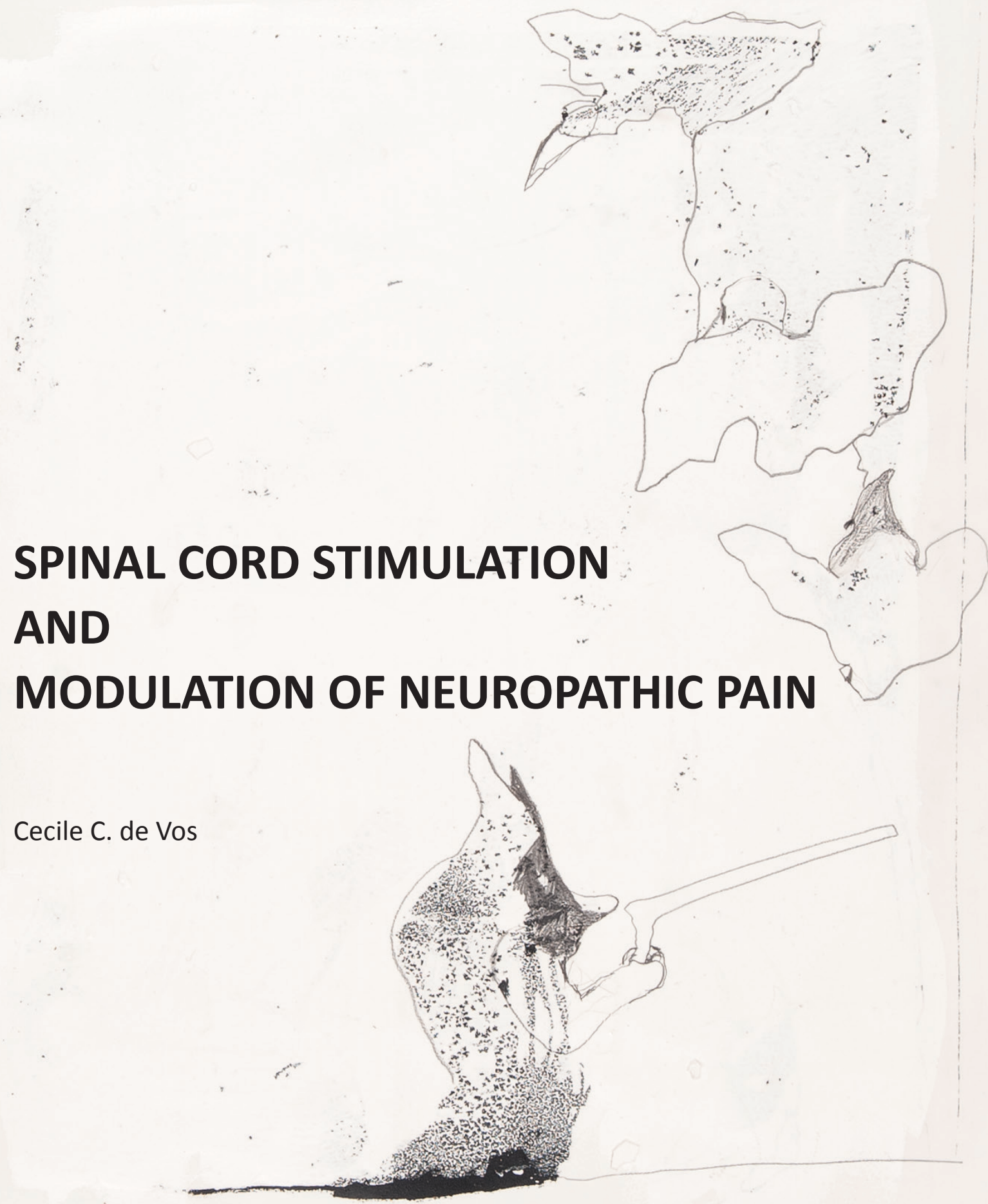




\title{
SPINAL CORD STIMULATION
}

\author{
AND \\ MODULATION OF NEUROPATHIC PAIN
}

Cecile C. de Vos 
The research presented in this thesis was done in the group Clinical Neurophysiology, MIRA institute for biomedical technology and technical medicine, University of Twente, the Netherlands and at the departments of Neurosurgery and Clinical Neurophysiology, Medisch Spectrum Twente hospital, the Netherlands.

Medtronic provided an unrestricted research grant to perform the studies presented in Chapter 2 and 7 and St Jude Medical sponsored the study presented in Chapter 4.

Cover: I tell you about my dreams 10-14, Sarah Grothus, www.sarahgrothus.nI Lay-out and printed by: Gildeprint Drukkerijen, Enschede, the Netherlands

ISBN 978-90-365-0256-6

DOI 10.3990/1.9789036502566

(c) C.C. de Vos, Enschede, the Netherlands, 2013 


\title{
SPINAL CORD STIMULATION
}

\section{AND \\ MODULATION OF NEUROPATHIC PAIN}

\author{
PROEFSCHRIFT
}

ter verkrijging van

de graad van doctor aan de Universiteit Twente,

op gezag van de rector magnificus,

prof. dr. H. Brinksma

volgens besluit van het College voor Promoties

in het openbaar te verdedigen op

donderdag 29 augustus 2013 om 14:45 uur

door

Cecilia Clementine de Vos

geboren op 23 december 1975

te Eindhoven 
Samenstelling promotiecommissie:

Voorzitter en Secretaris:

Prof.dr. G. van der Steenhoven Universiteit Twente

Promotor:

Prof.dr.ir. M.J.A.M. van Putten Universiteit Twente

Leden:

Dr. J. Holsheimer

Universiteit Twente

Prof.dr. F.J.P.M. Huygen

Erasmus Universiteit Rotterdam

Dr. K. Meier

Aarhus University, Denmark

Prof.dr. J.S. Rietman

Universiteit Twente

Prof.dr.ir. P. H. Veltink

Universiteit Twente

Prof.dr.ir. P.P.C.C. Verbeek

Universiteit Twente 


\section{TABLE OF CONTENTS}

$\begin{array}{lll}\text { Chapter 1: Introduction } & 7\end{array}$

Chapter 2: Effect and safety of spinal cord stimulation for treatment of chronic 17 pain caused by diabetic neuropathy

Chapter 3: Long-term effects of spinal cord stimulation in patients with painful diabetic neuropathy

Chapter 4: $\quad$ Spinal cord stimulation in patients with painful diabetic neuropathy: a multi centre randomised clinical trial

Chapter 5: Spinal cord stimulation with hybrid lead relieves pain in low back and legs

Chapter 6: Burst spinal cord stimulation evaluated in patients with failed back surgery syndrome and painful diabetic neuropathy

Chapter 7: Electrode contact configuration and energy consumption in spinal cord stimulation

Chapter 8: Pain evoked potentials in chronic pain patients with spinal cord stimulation

Chapter 9: $\quad$ Living with spinal cord stimulation 



\section{Chapter 1}

Introduction on Spinal Cord Stimulation and Neuropathic Pain 
8 | Chapter 1 


\section{INTRODUCTION}

\section{Definition of pain}

The International Association for the Study of Pain (IASP) defines pain as an "unpleasant sensory and emotional experience associated with actual or potential tissue damage, or described in terms of such damage" [IASP]. This definition emphasizes that pain is a highly subjective experience and does not need to have any obvious clinical signs or parameters that can be objectively measured. Pain can be influenced by memories, emotional, pathological, genetic and cognitive factors and is therefore not always related to the degree of tissue damage. This holds especially in patients with chronic pain. Chronic pain is pain that continues beyond the normal course of a disease or healing time of an injury [IASP]. Current estimates are that the prevalence of chronic non-malignant pain in Western European countries is about $18 \%$ which is accompanied by large socioeconomic costs [Wolff 2011, Eriksen 2003]. Chronic pain interferes significantly with the quality of life and general functioning of many people [Breivik 2006].

The IASP definition of pain emphasizes that pain is a subjective experience and does not need to have objectively measurable parameters. However, to study the effect of a pain therapy it is necessary to define parameters that can be measured in various patient groups and at various time points during treatment. Several questionnaires are available for this purpose (e.g. McGill Pain Questionnaire, EQ5D, Oswestry Disability Index, Visual Analogue Scale, Numeric Rating Scale, Short Form 36, Sickness Impact Profile) [Chapman 2011]. Since all reporting on pain is subjective, well informed participation of the patients is a prerequisite for every questionnaire.

The most common measure of pain intensity as perceived by a patient is the visual analogue scale (VAS): the patient indicates on a line the intensity of the pain perceived with $0 \mathrm{~mm}$ representing no pain to $100 \mathrm{~mm}$ representing the worst possible pain. A pain score on an 11-point numerical rating scale (NRS) provides a comparable measure, but now the patient is asked to rate his pain between 0 (no pain) and 10 (worst possible pain). An other commonly used questionnaires is the McGill pain questionnaire [Melzack 1987], which not only measures the pain intensity but also pain qualities and influence of the pain on daily activities. 


\section{Various types of pain}

Pain can be often subdivided into nociceptive pain, which is pain arising from actual or threatened damage to non-neural tissue and due to activation of nociceptors, and neuropathic pain, which is pain arising as a direct consequence of a lesion or disease affecting the somatosensory system [Treede 2008]. Neuropathic pain encompasses a wide variety of conditions including painful neuropathies, pain after damage to peripheral nerves or spinal nerve roots, deafferentiation pain, central post stroke pain, and complex regional pain syndrome. Unlike nociceptive pain, neuropathic pain is caused by dysfunction of the peripheral or central nervous or somatosensory system, and does not require any receptor stimulation.

\section{Failed back surgery syndrome}

Chronic radicular low back pain is probably the most prevalent pain syndrome to which neuropathic mechanisms contribute [Dworkin 2003]. However indentifying the neuropathic component is difficult as it is likely that a combination of skeletal, myofascial and neuropathic mechanisms account for this type of pain in many patients. Unfortunately, $10-40 \%$ of the patients with neuropathic radicular pain who have undergone spine surgery experience persistent or recurrent pain instead of alleviation [Kumar 2007]. This persistent pain of neuropathic and nociceptive origin is called failed back surgery syndrome (FBSS) and usually represents a combination of radicular pain in one or two legs and axial lower back pain.

\section{Painful diabetic neuropathy}

Neuropathy is one of the most common long-term complications of diabetes and a large study showed that in the United Kingdom one-third of all community-based diabetic patients has neuropathic pain [Abbott 2011]. Painful diabetic neuropathy (PDN) is typically more severe at night, often resulting in sleep disturbance and is associated with significant reduction in quality of life [Tesfaye 2011].

PDN generally starts in the toes and gradually progresses proximally. At a later stage, it may affect the hands as well, following the typical symmetrical 'glove and stocking' pattern. Although the exact pathophysiological mechanisms of neuropathic pain in diabetes remain unknown, several central and peripheral mechanisms have been postulated including increased blood glucose instability, altered foot skin microcirculation, reduced intraepidermal nerve fibre density, increased thalamic vascularity and autonomic dysfunction 
[Jensen 2003, Kapur 2003, Tesfaye 2005, Tesfaye 2011]. All these proposed mechanisms have in common that treatment options are limited: until the primary cause of the pain can be cured, therapies will need to target the pain symptoms.

\section{Neuromodulation to treat pain}

Spinal cord stimulation (SCS) is a type of neuromodulation device that is used as a last resort treatment for chronic pain caused by various types of neuropathy or failed back surgeries. $A$ neuromodulation device is invasive technology that acts directly upon neural tissue. In case of electrical neuromodulation, it causes modulation of neural activity through the delivery of electrical energy directly to a target area. Stimulation electrodes can be implanted in the brain, on the spinal cord, or on peripheral nerves and are connected to an implanted pulse generator via a subdermal extension cable. Neuromodulation works by stimulating the target area to produce biological responses that might have been disturbed or diminished because of a disease or a medical condition.

\section{Gate control theory}

Painful sensations, like other sensory information, are transported via sensory nerve fibres towards the brain. Unmyelinated C fibres transmit the slower component of pain, whereas small myelinated A $\delta$ fibres transmit the faster component. In 1965, Ronald Melzack and Patrick David Wall published "The Gate Control Theory of Pain" [Melzack 1965]. Central to the theory was the postulate that activation of large myelinated $A \beta$ fibres can diminish the transmission of pain activity transported by $A \delta$ and $C$ fibres via an integrative 'gate' in the spinal cord. Although the theory is most likely an oversimplification of the complex mechanisms that occur in the spinal cord and brain, it provided an initial theoretical framework for spinal cord stimulation as treatment for pain.

\section{Spinal cord stimulation}

SCS is an invasive treatment for chronic pain based on electrical stimulation of the dorsal columns of the spinal cord. To target the large myelinated $A \beta$ fibres in the dorsal column, an electrode lead is implanted in the epidural space and connected subcutaneously to an implanted pulse generator. The electrical stimulation at a level that corresponds to nerve fibres innervating the painful body area causes reduction of the perceived pain and elicits paraesthesia (tingling sensations) in most patients. When the stimulation amplitude is increased, mostly the intensity of the perceived paraesthesia increases and the covered 
body area enlarges, because an increased number of dorsal column fibres is recruited [Holsheimer \&Wesselink 1997]. Nevertheless, the therapeutic range of the stimulation amplitude is limited. Increasing stimulation amplitudes can be perceived as uncomfortable and even painful when not just dorsal column fibres, but also dorsal root fibres are activated [Holsheimer 1997]. Empirical evidence has shown that an optimal overlap of the perceived paraesthesia with the painful body area increases the chances to obtain good pain reduction with SCS.

The implantation of the spinal cord stimulation device comprises two phases. First, the electrode lead is implanted in the epidural space, either percutaneously via a modified epidural needle or via surgical laminectomy. The electrode is positioned over the dorsal column at the optimal level and connected to a temporary pulse generator outside the body. There is a consecutive trial period of several days. Only if the trial period is successful, generally that is, if a patient experiences more than $50 \%$ pain reduction, the external pulse generator will be converted into an implanted pulse generator. This pulse generator is implanted under the skin of the lower abdomen or upper buttock.

In the Netherlands, many of the implanted pulse generators are still non-rechargeable and have to be surgically replaced after some years (generally between three and seven). However, rechargeable SCS systems have become more common and clear the way for new stimulation paradigms that require more energy from the battery. Stimulation paradigms with multiple stimulation configurations or high frequencies require more energy from the battery, but provide more possibilities to optimize SCS for the individual patient's needs. The stimulation frequency is one of the parameters that can be adapted when programming the stimulation settings and for many patients it largely influences the perceived paraesthesia. Stimulation with frequencies below $30 \mathrm{~Hz}$ evokes more distinct tingling sensations, described as many tiny prickles/tickles, whereas stimulation with higher frequencies is generally experienced as a smoother sensation. New stimulation paradigms with intermittent or continuous stimulation frequencies of $500 \mathrm{~Hz}$ and above are believed not to cause any paraesthesia at all and still achieve good results [De Ridder 2010, De Ridder 2013, Van Buyten 2012]. 


\section{Indications for SCS}

SCS has shown to be an effective treatment for various neuropathic pain conditions, but the number of randomised controlled trials performed is limited [Mailis-Gagnon 2004]. Until now, indications that have been evaluated as amenable for SCS in randomised trials are complex regional pain syndrome (CRPS I)[Kemler 2008], radicular pain after failed back surgery (FBSS)[Kumar 2007, North 2005] and angina pectoris [De Jongste 1994, Mannheimer 1998]. Smaller studies and case reports on other conditions that have provided encouraging results with SCS include chronic visceral pain [Kapural 2010] and pain due to peripheral nerve injury [Kumar 1996] and diabetic neuropathy [Tesfaye 1996], but the efficacy of SCS remains undecided in pain conditions like phantom pain [Viswanathan 2010], postherpetic neuralgia [Kumar 1996] and critical limb ischaemia [Klomp 1999].

Nevertheless, even in carefully selected cases, there is a considerable percentage of patients (25-50\%, depending on the indication) who report diminished or loss of analgesic effect of SCS 6 to 24 months after initiation of the therapy [Simpson 2003, Sparkes 2012]. Attempts to identify clinical, psychological or demographical predictors for sustainable success have so far been unsuccessful. Still the strongest predictor of a negative SCS outcome on the long term is less then 50\% pain relief during the trial period [Williams 2011, Sparkes 2010]. Why stimulation does not provide (sustained) pain relief in a patient with neuropathic pain, but causes excellent pain relief in the next patient with the same diagnosis is still an unresolved problem of the field [Simpson 2003].

\section{Alterations in the central nervous system}

There is thus a need for improving the ability to identify underlying mechanisms of chronic neuropathic pain as well as predictive methods to support pain treatment decisions. Neuroimaging might be able to provide help in understanding the central mechanisms involved in pain processing. So far, imaging studies have demonstrated that chronic pain can alter brain functioning by structural remapping and functional reorganization of various brain areas and circuits [Kupers 2006, Tracey 2008, Saab 2012]. Imaging studies using positron emission tomography (PET) [Kishima 2010] and functional magnetic resonance imaging (fMRI) [Stancak 2008, Moens 2012] have also shown that SCS can alter the activity in a large number of brain regions, including thalamus, secondary somatosensory cortex, inferior temporal cortex, cerebellar cortex, primary motor cortex, cingulated cortex, prefrontal cortex and insula. However, thus far there is only limited and even partly contradicting 
evidence available, so no attempts have been made at assessing the role and importance of each region yet.

\section{Thesis outline}

This thesis will report on the opportunities of several new applications of spinal cord stimulation for the treatment of neuropathic pain and pain after failed back surgery. Chapter 2 and 3 report our pilot study on short-term and long-term effects of spinal cord stimulation on pain intensity and quality of life in patients with painful diabetic neuropathy. The results from this pilot study motivated the initiation of an international multi centre randomised clinical trial. The first results from this clinical trial are presented in Chapter 4. Clinical evaluations of novel SCS hardware (percutaneous paddle leads) and SCS software (burst stimulation) are presented in Chapters 5 and 6 . As new stimulation paradigms require more energy than conventional tonic stimulation, also patients with a rechargeable pulse generator will benefit from programming stimulation configurations that require least energy (Chapter 7). When programming stimulation settings to optimize pain relief, we strongly rely on information provided by the patients. There are no objective measures for pain perception available. In Chapter 8 we show however that we might be able to measure cortical alterations in pain processing in patients who respond favourably to spinal cord stimulation. Finally, in chapter 9, the impact of living with spinal cord stimulation is discussed. Contemporary philosophical frameworks for apprehending human-technology relations are explored to understand the intimate relation with a technological device a patient is entering when a spinal cord stimulator is implanted. 


\section{REFERENCES}

Abbott CA et al., Prevalence and Characteristics of Painful Diabetic Neuropathy in a Large Community-Based Diabetic Population in the U.K., Diabetes Care 2011; 34:2 220-2224

Breivik H, Collett B, Ventafridda V, Cohen R, Gallacher D. Survey of chronic pain in Europe: Prevalence, impact on daily life, and treatment. Eur J Pain 2006; 10: 287-333

Chapman JR, Norvell DC, Hermsmeyer JT, Bransford RJ, DeVine J, McGirt MJ, Lee MJ. Evaluating Common Outcomes for Measuring Treatment Success for Chronic Low Back Pain. Spine 2011; 36: s54-s68

de Jongste MJ, Hautvast RW, Hillege HL, Lie KI. Efficacy of spinal cord stimulation as adjuvant therapy for intractable angina pectoris: a prospective, randomized clinical study. Working Group on Neurocardiology. J Am Coll Cardiol 1994; 23: 1592-1597

de Ridder D, Vanneste S, Plazier M, van der Loo E, Menovsky T. Burst Spinal Cord Stimulation: Toward ParesthesiaFree Pain Suppression. Neurosurgery 2010: 66; 986-990

de Ridder D, Plazier M, Kamerling N, Menovsky T, Vanneste S. Burst Spinal Cord Stimulation for Limb and Back Pain. World Neurosurg 2013. doi: 10.1016/j.wneu.2013.01.040

Dworkin R, et al. Advances in neuropathic pain: diagnosis mechanisms and treatment recommendations. Arch Neurol 2003; 60: 1524-1534

Eriksen J, Jensen MK, Sjogren P, Ekholm O, Rasmussen NK. Epidemiology of chronic non-malignant pain in Denmark. Pain 2003; 106: 221-228.

Holsheimer J. Effectiveness of spinal cord stimulation in the management of chronic pain: analysis of technical drawbacks and solutions. Neurosurgery 1997; 40: 990-999

Holsheimer J, Wesselink WA. Effect of anode-cathode configuration on paresthesia coverages in spinal cord stimulation. Neurosurgery 1997; 41: 654-660

IASP Task Force on Taxonomy, Part III: Pain Terms, A Current List with Definitions and Notes on Usage, in: Classification of chronic pain, ed. H. Merskey and N. Bogduk, $2^{\text {nd }}$ edition, IASP Press, Seattle, 1994, $209-214$.

Jensen TS, Baron R. Translation of symptoms and signs into mechanisms in neuropathic pain. Pain 2003; 102: 1-8.

Kapur D. Neuropathic pain and diabetes. Diabetes Metab Res Rev 2003; 19: S9-S15

Kapural L, Nagem H, Tlucek H, Sessler DI. Spinal cord stimulation for chronic visceral abdominal pain. Pain Med 2010; 11: 347-355.

Kemler MA, de Vet HC, Barendse GA, van den Wildenberg FA, van KM. Effect of spinal cord stimulation for chronic complex regional pain syndrome Type I: five-year final follow-up of patients in a randomized controlled trial. J Neurosurg 2008; 108: 292-298

Kishima H, Saitoh Y, Oshino S et al. Modulation of neuronal activity after spinal cord stimulation for neuropathic pain; $\mathrm{H}(2) 150$ PET study. Neuroimage 2010; 49 :2564-2569

Kumar K, Taylor RS, Jacques L et al. Spinal cord stimulation versus conventional medical management for neuropathic pain: a multicentre randomised controlled trial in patients with failed back surgery syndrome. Pain 2007; 132: 179-188.

Kupers $\mathrm{R}$, Kehlet $\mathrm{H}$, Brain imaging of clinical pain states: a critical review and strategies for future studies, Lancet Neurol 2006; 5: 1033-44

Mailis-Gagnon A, Furlan AD, Sandoval JA, Taylor RS. Spinal cord stimulation for chronic pain. Cochrane Database of Systematic Reviews 2004, Issue 3. Art. No.: CD003783. DOI: 10.1002/14651858.CD003783.pub2.

Mannheimer C, Eliasson T, Augustinsson LE et al. Electrical stimulation versus coronary artery bypass surgery in severe angina pectoris: the ESBY study. Circulation 1998; 97: 1157-1163.

Meier K, Spinal cord stimulation: Clinical and experimental aspects, PhD dissertation, Aarhus University, 2012

Melzack R. The short-form McGill Pain Questionnaire. Pain 1987; 30: 191-197

Melzack R, Wall PD. Pain mechanisms: a new theory. Science 1965; 150: 971-979

North RB, Kidd DH, Farrokhi F, Piantadosi SA. Spinal cord stimulation versus repeated lumbosacral spine surgery for chronic pain: a randomized, controlled trial. Neurosurgery 2005; 56: 98-106

Simpson BA. Selection of patients and assessment of outcome. In: Simpson BA, editor. Electrical stimulation and the relief of pain. Pain research and clinical management vol.15, Amsterdam: Elsevier; 2003 pp.237-249 
Sparkes E, Raphael JH, Duarte RV, Lemarchand K, Jackson C and Ashford RL. A systematic literature review of psychological characteristics as determinants of outcome for spinal cord stimulation therapy. Pain 2010; 150: 284-289.

Sparkes E, Duarte RV, Raphael JH, Denny E, Ashford RL, Qualitative exploration of psychological factors associated with spinal cord stimulation outcome, Chronic Illness 2012; 8: 239-251

Stancak A, Kozak J, Vrba I et al. Functional magnetic resonance imaging of cerebral activation during spinal cord stimulation in failed back surgery syndrome patients. Eur J Pain 2008; 12: 137-148.

Tesfaye S, Watt J, Benbow SJ, Pang KA, Miles J, MacFarlane IA. Electrical spinal cord stimulation for painful diabetic peripheral neuropathy. Lancet 1996; 348: 1698-1701.

Tesfaye S, Kempler P, Painful diabetic neuropathy, Diabetologia 2005; 48: 805-807

Tefaye S, Vileikyte L, Rayman G, Sindrup SH, Perkins BA, Baconja M, Vinik Al, Boulton AJM. Painful diabetic peripheral neuropathy: consensus recommendations on diagnosis, assessment and management, Diabetes Metab Res Re.v 2011; 27: 629-638

Tracey I, Imaging pain, British Journal of Anaesthesia 2008; 101: 32-9

Treede RD et al., Neuropathic pain Redefinition and a grading system for clinical and research purposes, Neurology 2008; 70

van Buyten JP, Al-Kaisy A, Smet I, Palmisani S, Smith T. High-Frequency Spinal Cord Stimulation for the Treatment of Chronic Back Pain Patients: Results of a Prospective Multicenter European Clinical Study. Neuromodulation 2012. doi: 10.1111/ner.12006

Viswanathan A, Phan PC, Burton AW. Use of spinal cord stimulation in the treatment of phantom limb pain: case series and review of the literature. Pain Pract 2010; 10:479-484

Williams KA, Gonzalez-Fernandez M, Hamzehzadeh S, Wilkinson I, Erdek MA, Plunkett A, Griffith S, Crooks M, Larkin T, Cohen SP, A Multi-Center Analysis Evaluating Factors Associated with Spinal Cord Stimulation Outcome in Chronic Pain Patients, Pain Medicine 2011; 12: 1142-1153

Wolff R, Clar C, Lerch C, Kleijnen J, Epidemiologie von nicht tumorbedingten chronischen Schmerzen in Deutschland, Schmerz 2011 25:26-44 


\section{Chapter 2}

\section{Effect and safety of spinal cord stimulation for}

\section{treatment of chronic pain caused by diabetic}

\section{neuropathy}

Cecile C de $\operatorname{Vos}^{1}$, Vinayakrishnan Rajan ${ }^{3}$, Wiendelt Steenbergen ${ }^{3}$, Hans E van der $\mathrm{Aa}^{1,2}$, Hendrik PJ Buschman ${ }^{1,4}$

${ }^{1}$ Twente Institute for Neuromodulation, Medisch Spectrum Twente, Enschede, The Netherlands

${ }^{2}$ Department of Neurosurgery, Medisch Spectrum Twente, Enschede, The Netherlands

${ }^{3}$ Department of Biophysical Engineering, University of Twente, Enschede,

The Netherlands

${ }^{4}$ Department of Biomedical Signals and Systems, University of Twente, Enschede,

The Netherlands

Journal of Diabetes and Its Complications. 2009; 23:40-45 


\section{ABSTRACT}

Aim: Spinal cord stimulation (SCS) has been shown effective as a therapy for different chronic painful conditions, but the effectiveness of this treatment for pain as a result of peripheral diabetic neuropathy is not well established. The primary objectives of this study were to evaluate the effect and safety of SCS for treatment of pain and the effects on microcirculatory blood flow in the affected areas in patients with refractory peripheral diabetic neuropathy.

Method: The study was designed as a prospective, open-label study. Data were collected during screening, at implant and at regular intervals, after initiation of therapy. Eleven diabetic patients with chronic pain in their lower limbs and no response to conventional treatment were studied. The SCS electrode was implanted in the thoracic epidural space. Neuropathic pain relief was assessed by Visual Analogue Scale (VAS) and microcirculatory skin perfusion was measured with Laser Doppler flowmetry.

Results: Nine patients had significant pain relief with the percutaneous electrical stimulator. Average pain score for all nine patients was 77 at baseline and 34 at 6 months after implantation. At the end of the study, eight out of nine patients continued to experience significant pain relief and were able to significantly reduce their pain medication. For six of them, the stimulator was the sole treatment for their neuropathic pain. No significant changes in microcirculatory perfusion were recorded.

Conclusion: Spinal cord stimulation offers an effective and safe therapy for chronic diabetic neuropathic pain. 


\section{INTRODUCTION}

Electrical spinal cord stimulation (SCS) has been shown effective as a therapy for different chronic painful conditions, but the effect of this treatment for pain as a result of peripheral diabetic neuropathy is not well established. In literature, the information about this therapy in this patient group is sparse.

Few studies to date have concentrated on the use of SCS in peripheral neuropathy [Kavar 2000; Kumar 1996; Daousi 2004; North 1993; Tesfaye 1996]. Most studies involved sources of peripheral neuropathic pain of mixed aetiology and therefore do not possess adequate descriptions of the effectiveness of SCS for diabetic peripheral neuropathy specifically. The research carried out by Tesfaye et al. in 10 diabetic patients showed that eight patients responded well to a percutaneous electrical stimulator and were converted to a permanent stimulator. At 6 and 14 months, significant pain relief was achieved in seven patients. Recently, a follow-up study showed that after 3 years $(n=6)$ and after 7 years $(n=4)$, there was still significant pain relief [Daousi 2004]. Similarly Kumar et al. have investigated the long-term results in a group of 30 patients with peripheral neuropathy. All patients used medication to reduce the neuropathic pain prior to spinal cord stimulation and underwent several other therapies without sufficient pain relief. Of the 30 patients included, four patients had a peripheral neuropathy due to diabetes. All four diabetic patients had good to very good results in short-term and three in long-term (36-149 months).

The pathophysiology of painful diabetic neuropathy is complex and multifactorial [Gooch 2004]. One of the important factors is impaired blood supply to the peripheral nerves. Patients with diabetic neuropathy would greatly benefit from an increase in perfusion in the affected areas. Ghajar \& Miles [1998] have performed measurements on patients with peripheral vascular diseases in the lower limbs. They found that the position of the SCS electrode influences the effect on capillary blood flow in the affected limbs and suggest that this is due to the segmental nature of the sympathetic nervous system. Studies in rats have shown that SCS antidromically activates afferent fibres in the dorsal root, causing peripheral release of peptide CGRP and, consequently, cutaneous vasodilatation [Tanaka 2004]. 
A complete understanding, however, of the possible mechanisms causing a change in microcirculatory blood flow is not yet achieved. Nevertheless, some other studies show that, in patients with neuropathic pain and complex regional pain syndrome, pain relief experienced from SCS is not related to changes of skin blood flow [Ather 2003; Kemler 2000]. However, these measurements have not been done on patients with diabetic neuropathy. A study with diabetic patients with neuropathy and severe stages of peripheral vascular disease (PVD) has shown that diabetic patients with advanced PVD receive only limited benefit from SCS [Petrakis 1999; 2000]. We expected that applying SCS to patients with diabetic neuropathy with less severe circulatory problems could cause not only good pain relief but also increased skin blood microcirculation. The primary objectives of this study were to evaluate the effect and safety of spinal cord stimulation for treatment of pain and the effects on microcirculatory blood flow in patients with refractory peripheral diabetic neuropathy.

\section{METHODS}

\section{Patients}

Since 2004, 11 diabetic patients diagnosed with chronic neuropathic pain who did not respond to conventional treatment were included in the study. They were nine men and two women: mean age, 63 (SD 8) years; mean duration of diabetes, 19 (14) years; and mean duration of painful diabetic neuropathy, 9 (5) years. All patients had good glucose control and were referred to our group by their treating specialist. In all patients, painful neuropathy was situated in their lower limbs, and none of them had trophic lesions. Two patients had initial signs of neuropathy in the upper limbs. All patients have been using medicationlike antidepressants, anticonvulsants, and opioids for years and underwent several other therapies like transcutaneous electronic nerve stimulation, physical therapy, and therapy to help coping with pain, attempting to alleviate their neuropathic pain. At the start of our study, three patients used oral opioids, one patient used clonazepam, and all other patients used gabapentin (1600-3000 mg/day). Occasionally, other types of pain medication such as nonsteroidal anti-inflammatory drugs were used in combination with these. However, none of the 11 patients experienced sufficient pain relief from the medication they used. 


\section{Pain}

All patients suffered from sensorimotor polyneuropathy and felt burning and pricking pain in their lower limbs. The pain was almost always present and, in most patients, intensified during standing or walking.

Patients were asked to rate their overall pain on a Visual Analogue Scale (VAS) in the morning, afternoon, evening, and night for a period of 7 days and supplied information about the medication they used for their neuropathic pain. These data were collected at baseline; during screening; at implantation; and at 1, 3, and 6 months after initiation of therapy. During screening and after 6 months, a structured interview was performed as well. The average pain as measured by VAS and the patient global impression of change (PGIC) [Farrar 2001] at 6 months were the primary end point parameters of this pilot study. Additionally, after 12 and 30 months, the patients were interviewed and asked to rate their pain again.

\section{Treatment protocol}

The treatment protocol has been approved by the responsible medical ethics committee and is based on the model for treatment of chronic noncancerous pain with spinal cord stimulation, which was developed by the Dutch Neuromodulation study group in 2001 [De Bruijn 2002]. Informed consent of all participating patients had been obtained. Only patients with refractory neuropathic pain due to diabetes were included. Patients with severe stages of vascular disease (Fontaine Stages IV and V) or with pain due to other causes than neuropathy were excluded from the study.

In conformity to the Dutch Neuromodulation Study Group protocol, a quadripolar electrode lead (Medtronic, Minneapolis, MN, USA) was implanted in the thoracic epidural space via a percutaneous lumbar puncture. The single lead, containing four electrode contacts, was positioned medially over thoracic segments of the spinal cord according to the patient's description of the paraesthesia.

Before a permanent stimulator was implanted, a test period of a week with a percutaneously implanted externalised lead, and a handheld external pulse generator was performed to find out whether the patient achieved sufficient pain relief from spinal cord stimulation. When a patient reacted positively to the test stimulation (>30 points pain reduction on a 100-point VAS), a permanent stimulator was implanted (Itrel 3 or Synergy, Medtronic). 
During the test period and with the permanent stimulator, the patient was able to switch on and off the stimulation and adjust the intensity of the stimulation pulses to the level required at any moment (e.g., sitting, lying, or standing/walking). The combination of active electrodes, pulse width, pulse frequency, and upper and lower intensity boundaries for the individual patients were programmed into the pulse generator by the physician assistant.

\section{Perfusion}

The blood flow of the skin was measured using laser Doppler flowmetry technique. It is a noninvasive technique to monitor the skin microcirculation with a typical measurement depth of 1-2 $\mathrm{mm}$. It is based on the induced Doppler shift of light scattered from moving blood cells. A monochromatic beam of light is delivered to an area of tissue with an optical fibre. The light back-scattered from moving blood cells undergoes a change in wavelength (Doppler shift). The back-scattered light is collected with a detection fibre. The relative amount of Doppler shifted photons and their mean Doppler shift are directly related to the concentration and root mean square velocity of red blood cells in the exposed area of tissue [Bonner 1981]. The perfusion is represented in absolute perfusion units (p.u.), which is the flux of moving blood cells in tissue.

The measurements were performed using a commercial laser Doppler perfusion monitor (PF5000, Perimed, Sweden). The instrument has a semiconductor laser diode of $780 \mathrm{~nm}$ and a Doppler bandwidth of $20 \mathrm{~Hz}-13 \mathrm{kHz}$. Two standard probes (Probe 408, Perimed) were used in the experiment. The probe has illumination and detection fibres at $250 \mu \mathrm{m}$ mutual distance, with a core diameter of $125 \mu \mathrm{m}$ and a numerical aperture of 0.37 . The laser Doppler instrument was allowed to warm up for 20 min before the start of the base measurements. The device in combination with two probes was calibrated using a motility standard (PF 1000, Perimed). Both probes were attached to the measurement site with a probe holder and sticker. The time constant of the output signals was set at $0.2 \mathrm{~s}$. Perfusion measurements were taken at a sampling rate of $32 \mathrm{~Hz}$.

Perfusion levels were acquired from two positions on the affected part of the body where a good paraesthesia was experienced. The same two positions were used for all measurements on the single patient. The average time of a measurement was $10 \mathrm{~min}$. During all measurements, the patients were in a comfortable horizontal position and the temperature in the room was kept stable between $20^{\circ} \mathrm{C}$ and $22^{\circ} \mathrm{C}$. The measurements were 
performed around noon, and the patients were asked not to use tobacco, coffee, and tea during the morning prior to the measurements. The average microcirculatory blood flow was measured before implantation and 1, 3, and 6 months after initiating spinal cord stimulation.

\section{RESULTS}

Nine subjects with mean age of 61 (SD 11) years, mean duration of diabetes of 21 (14) years, and mean duration of neuropathy of 10 (5) years had significant pain relief with the percutaneous electrical stimulator and were converted to a permanent system (Table 1). Two patients did not benefit from the percutaneous test stimulation and therefore did not receive a permanent stimulator. The results from the nine patients are presented.

Table 1 Characteristics of the patients who have received a permanent stimulator.

\begin{tabular}{llllllllll}
\hline Patient & 1 & 2 & 3 & 4 & 5 & 6 & 7 & 8 & 9 \\
Gender & $\mathrm{M}$ & $\mathrm{F}$ & $\mathrm{M}$ & $\mathrm{M}$ & $\mathrm{F}$ & $\mathrm{M}$ & $\mathrm{M}$ & $\mathrm{M}$ & $\mathrm{M}$ \\
Age & 49 & 59 & 72 & 59 & 66 & 54 & 73 & 74 & 45 \\
Diabetes type & $\mathrm{II}$ & $\mathrm{I}$ & $\mathrm{I}$ & $\mathrm{II}$ & $\mathrm{I}$ & $\mathrm{I}$ & $\mathrm{I}$ & $\mathrm{II}$ & $\mathrm{I}$ \\
Diabetes duration (y) & 7 & 50 & 10 & 14 & 26 & 8 & 24 & 15 & 35 \\
Pain duration (y) & 5 & 20 & 4 & 12 & 7 & 12 & 5 & 7 & 10 \\
\hline
\end{tabular}

\section{Pain}

More than $50 \%$ relief of neuropathic pain was experienced at 1 month by five patients, and one patient experienced $30-50 \%$ pain relief. At 3 and 6 months, more than $50 \%$ pain relief was achieved in six patients and $30-50 \%$ pain relief in two patients (Figure 1 and Table 2). In two patients, the connection between the lead and the extension cable was inadequate, and therefore, after 1 month a surgical revision was performed. After the revision the pain relief reappeared. Average pain score for all nine patients was 77 at baseline and 34 at 6 months after implantation. Although the primary outcome of our study was VAS and PGIC measured after 6 months of SCS, we have also assessed VAS after 12 months and VAS and PGIC after 30 months of SCS. After 12 months, in six patients, relief of neuropathic pain was even larger than after 6 months. The average pain score was 23 . Seven patients had more than $50 \%$ pain relief, and one patient had $30-50 \%$ pain relief. After 30 months, the average pain score was still 23 . Six patients still had more than $50 \%$ pain relief, and one patient had $30-50 \%$ pain relief. One patient died due to causes not related to SCS. 


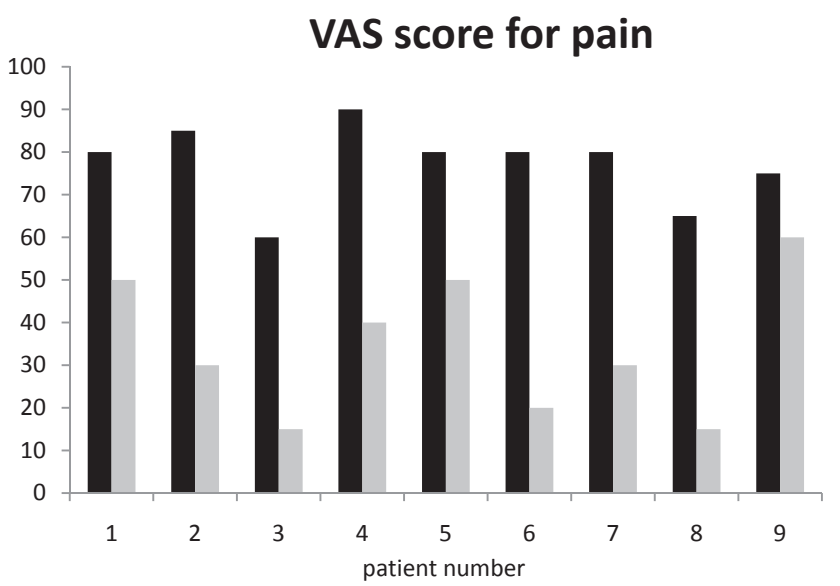

Figure 1 VAS pain scores of patients at baseline (black bars) and after 6 months (gray bars) of spinal cord stimulation treatment.

Table 2 For each patient the acquired VAS scores (average of 7 days), PGIC, perfusion (in p.u.), and the level of the active electrodes over the thoracic segments of the spinal cord.

\begin{tabular}{llllllllll}
\hline Patient & 1 & 2 & 3 & 4 & 5 & 6 & 7 & 8 & 9 \\
VAS baseline & 80 & 85 & 60 & 90 & 80 & 80 & 80 & 75 & 65 \\
VAS 6 months & 50 & 30 & 15 & 40 & 50 & 20 & 30 & 60 & 15 \\
VAS 12 months & 0 & 0 & 10 & 60 & 10 & 10 & 20 & 65 & 30 \\
VAS 30 months & 0 & 0 & 30 & 30 & 20 & 10 & - & 60 & 30 \\
PGIC 6 months & 1 & 1 & 2 & 2 & 2 & 2 & 1 & 3 & 1 \\
PGIC 30 months & 1 & 1 & 2 & 1 & 3 & 2 & - & 3 & 1 \\
Perfusion baseline & 7.5 & 7.9 & 14.0 & 4.9 & 11.9 & 31.9 & 5.7 & - & - \\
Perfusion 6 months & 20.9 & 6.7 & 10.9 & 8.2 & 11.5 & 33.2 & 9.8 & - & - \\
Electrode position & $\mathrm{t} 10-11$ & $\mathrm{t} 10-11$ & $\mathrm{t} 10-11$ & $\mathrm{t} 10-11$ & $\mathrm{t} 9-10$ & $\mathrm{t} 10-11$ & $\mathrm{t} 10-11$ & $\mathrm{t} 11-12$ & $\mathrm{t} 10-11$ \\
\hline
\end{tabular}

During the interview after 6 months, the patients were asked to evaluate their overall status compared to the beginning of the study and indicate their general impression of change. Eight patients chose the categories "1: very much improved" or "2: much improved." One patient chose "3: minimally improved." After 30 months, six patients still indicated their change in Category 1 or 2 . Two patients indicated to experience only minimal improvement. Further analysis showed that this was mainly due to increasing neuropathic pain in their upper limbs. Pain in the upper extremities cannot be treated with the electrode implanted at the thoracic level of the spinal cord. 


\section{Medication}

As a result of spinal cord stimulation, eight of nine patients have been able to reduce their pain medication significantly, and for six of them, the stimulator had become the sole treatment for their neuropathic pain. The three patients who used oral opioids did not use any medication anymore at 6 months of SCS treatment. Of the five patients who used gabapentin, only two still used it at a low dose (400 mg/day). The patient who used clonazepam still needed to use the same amount of medication.

\section{Perfusion}

Five of the nine patients and also their spouses mentioned that they experienced an apparent increase in temperature in their affected and treated limbs, suggesting a possible increase in perfusion. In seven patients with a permanent stimulator, the microcirculatory perfusion has been measured with laser Doppler flowmetry. Every time the patient visited the clinic, two consecutive perfusion measurements were performed within a period of $30 \mathrm{~min}$. The perfusion results over time are shown in Figure 2. Perfusion levels measured while two patients had a bad connection between lead and extension were excluded from analysis. The perfusion levels of each individual patient at baseline and after 6 months are presented in Table 2. The results show that although the average microcirculatory perfusion of the seven patients tended to increase, no statistical significant changes have been measured with laser Doppler flowmetry.

\section{Relation pain and perfusion}

In seven patients, VAS scores and microcirculatory perfusion have been acquired after 6 months of SCS. These seven patients had all improvements of VAS scores ranging from 30 up to 60 points. At the same time, only three patients showed a distinct increase in skin perfusion, two patients had no clear change, and two had a decrease in skin perfusion. In these seven patients, no correlation has been found between absolute perceived pain relief and relative change in blood perfusion after 6 months. 


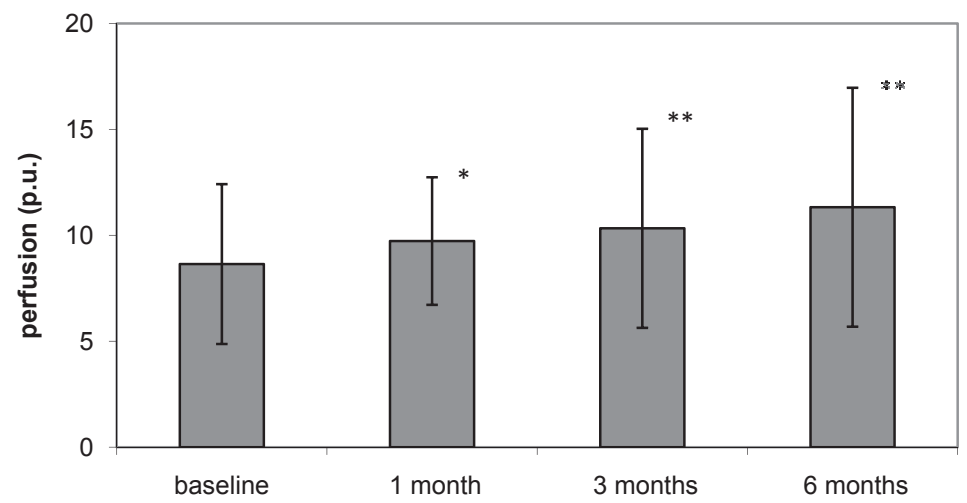

Figure 2 Perfusion (average of all patients and two measurements per time period) at baseline and after 1, 3 and 6 months of using SCS. The change in perfusion after 1, 3 and 6 months with respect to baseline perfusion is not significant. $* \mathrm{P}=0.7$ and ${ }^{* *} \mathrm{P}=0.3$.

\section{DISCUSSION}

At the end of the study, all patients with a permanent spinal cord stimulator continued to experience pain relief, and eight of nine patients have been able to reduce their pain medication. For six of them, the stimulator is the sole treatment for their neuropathic pain. Therefore, chronic diabetic neuropathic pain seems to be a favourable condition for treatment by spinal cord stimulation. These findings are in agreement with results from other studies on SCS in diabetic neuropathy that reported a 70-90\% success rate in obtaining $>50 \%$ pain reduction up to 1 year post implantation [Daousi 2004; Kumar 1996; Tesfaye 1996].

A weakness of primary endpoint of this study was the relative short follow-up period of 6 months because it has been argued that this period might represent a placebo effect. However, the long-term follow-up of these patients demonstrates that the pain suppressive effect of SCS remains or even increases. This long-term effect will be determined in a new and large randomised controlled trial currently being planned.

Contrary to studies from Claeys [2000], Petrakis [1999; 2000] and Ubbink [1999] that showed a correlation between pain relief and microcirculatory perfusion improvements, the patients we have been studying showed no correlation between perceived pain relief and change in perfusion of the skin after 6 months. It is possible that the laser Doppler methodology 
adopted by us was not the best technique for this purpose. It could have been better to use the laser Doppler technique to study the response of the vascular bed in, for instance, a post occlusive reactive hyperaemia provocation. However, it appeared to be impossible in many patients to measure this response due to the pain caused by the occlusion.

Eight out of nine patients in our study have changed their medication as a result of SCS therapy. Blood perfusion could be altered not only by SCS therapy but by changing drugs as well. However, none of the drugs used by the patients is associated with influencing the vascular system or microcirculatory blood flow. Still, several other factors may have affected the level of perfusion of the skin. As only perfusion in a superficial volume of a few $\mathrm{mm}^{3}$ is measured, there is a risk of choosing a non-representative point on the skin every time a measurement is performed and alterations in perfusion in the deeper vessels in the skin are not detected at all. In future studies, it may be beneficial to use techniques complimentary to laser Doppler flowmetry to overcome the limitations of this technique. We suggest the transcutaneous oxygen tension method to measure the amount of nutritional blood [Petrakis 1999; 2000] and infrared thermography to quantify the temperature changes on the lower legs.

According to Ghajar \& Miles [1998], the level of stimulation of the spinal cord causes either an increase or a decrease of the microvascular perfusion measured by laser Doppler flowmetry. As suggested by these authors, stimulation at level T8-T10 could show a tendency to decrease perfusion, while stimulation at level T12 could more likely have provoked an increase in perfusion. We have not been able to reproduce these findings, possibly due to too little variation in stimulation levels. In our study, the electrodes were positioned corresponding to the patient's description of the paraesthesia and maximal pain relief during the placement of the stimulation lead. As shown in Table 2, seven patients have the active electrodes positioned at thoracic level 10 and 11 , one patient has them placed at thoracic level 11 and 12, and one patient at thoracic level 9 and 10. Although there is only very little difference in level of the electrodes among the nine patients, both perfusion improvement and pain reduction differ to a great extent.

Although the average microcirculatory perfusion in the seven patients seemed to increase, no significant changes have been measured with laser Doppler flowmetry. This might be due to the large variation in responses of the patients. To acquire significant data about changes 
in microcirculatory perfusion, a larger study population is necessary. In our future study, a larger number of patients with diabetic neuropathic pain will be included, and they will be randomised to either a treatment group or a control group. The nonsignificant change in perfusion in this study also contradicts the patient's subjective blood perfusion reports. Five of the nine patients and their spouses have mentioned that they experienced an apparent change in temperature in their affected and treated limbs. However, we have not been able to demonstrate this effect with an objective measurement.

No structured questionnaire about daily activities or tests on walking distance has been assessed in this study; therefore, we have no objective measure for changes in mobility of these patients. Although eight of the treated patients have mentioned an increase in their mobility after 6 months, these remarks cannot be statistically related to either the pain scores or the perfusion measurements. For that reason, in our future randomised controlled trial, we will assess measurements on more areas like physical and emotional functioning as well, as advised by the Initiative on Methods, Measurement, and Pain Assessment in Clinical Trials (IMMPACT) recommendations [Turk 2003].

\section{Safety}

Due to an inadequate connection between the lead and the extension cable, a surgical revision was performed on two patients. After revision the pain relief reappeared in both patients. One patient has had a mild infection that has been treated easily with antibiotics, without any influence on the SCS treatment. No other adverse events have been recorded. The SCS treatment seems to be safe for patients with diabetic neuropathy.

In conclusion, SCS showed a statistically significant effect on chronic diabetic neuropathic pain, indicating that SCS could be considered as a clinically effective therapy for this patient group. However, our results do not come to conclusions about effects of SCS on microcirculatory blood flow. Furthermore, it was found that SCS is a safe therapy for treatment of diabetic neuropathic pain. The therapy could be considered in diabetic patients with neuropathic pain who do not respond to conventional treatment.

\section{Acknowledgments}

The authors would like to acknowledge Perimed for providing a laser Doppler perfusion monitor for the measurements and Medtronic for an unrestricted research grant. 


\section{REFERENCES}

Ather, M., di Vadi, P., Light, D., Wedley, J. R., \& Hamann, W. C. (2003).Spinal cord stimulation does not change peripheral skin blood flow in patients with neuropathic pain. European Journal of Anaesthesiology, 20, 736-739.

Bonner, R. F., \& Nossal, R. (1981). Model for laser Doppler measurements of blood flow in tissue. Applied Optics, 20, 2097-2107.

Claeys, L. G. Y. (2000). Pain relief and improvement of nutritional skin blood flow under spinal cord stimulation in patients with limbthreatening ischemia. Pain Clinic, 12, 39-46.

Daousi, C., Benbow, S. J., \& MacFarlane, I. A. (2004). Electrical spinal cord stimulation in the long-term treatment of chronic painful diabetic neuropathy. Diabetic Medicine, 22, 393-398.

de Bruijn, J. H. B., Beersen, N., Bruijnzeels, M. A., Dekkers, M. A., Harteloh, P. P. M., ten Have, P., Hekster, G. B., Redekop, W. K., \& Klazinga, N. S. (2002). Eindrapportage Neuromodulatie bij de behandeling van patiënten met chronische niet-oncologische pijn. Amstelveen: College van zorgverzekeringen; 2002.

Farrar, J. T., Young, J. P., LaMoreaux, L., Werth, J. L., \& Poole, R. M. (2001). Clinical importance of change in chronic pain intensity measured on an 11-point numerical pain rating scale. Pain, 94, 149-158.

Ghajar, A. W., \& Miles, J. B. (1998). The different effect of the level of spinal cord stimulation on patients with advanced peripheral vascular disease in the lower limbs. British Journal of Neurosurgery, 12, 402-408.

Gooch, C., \& Podwall, D. (2004). The diabetic neuropathies. Neurologist, 10, 311-322.

Kavar, B., Rosenfeld, J. V., \& Hutchinson, A. (2000). The efficacy of spinal cord stimulation for chronic pain. Journal of Clinical Neuroscience, 7, 409-413.

Kemler, M. A., Barendse, G. A. M., van Kleef, M., \& Oude Egbrink, M. G. A. (2000). Pain relief in complex regional pain syndrome due to spinal cord stimulation does not depend on vasodilation. Anesthesiology, 92, 1653-1660.

Kumar, K., Toth, C., \& Nath, R. K. (1996). Spinal cord stimulation for chronic pain in peripheral neuropathy. Surgical Neurology, 46, 363-369.

North, R. B., Kidd, D. H., Zahurak, M., James, C. S., \& Long, D. M. (1993). Spinal cord stimulation for chronic, intractable pain: Experience over two decades. Neurosurgery, 32, 384-395.

Petrakis, I. E., \& Sciacca, V. (1999). Epidural spinal cord electrical stimulation in diabetic critical lower limb ischemia. Journal of Diabetes and Its Complications, 13, 293-299.

Petrakis, I. E., \& Sciacca, V. (2000). Spinal cord stimulation in diabetic lower limb critical ischaemia: Transcutaneous oxygen measurement as predictor for treatment success. European Journal of Vascular and Endovascular Surgery, 19, 587-592.

Tanaka, S., Komori, N., Barron, K. W., Chandler, M. J., Linderoth, B., \& Foreman, R. D. (2004). Mechanisms of sustained cutaneous vasodilatation induced by spinal cord stimulation. Autonomic Neuroscience, 114, 55-60.

Tesfaye, S., Watt, J., Benbow, S. J., Pang, K. A., Miles, J., \& MacFarlane, I. A. (1996). Electrical spinal-cord stimulation for painful diabetic peripheral neuropathy. Lancet, 348, 1698-1701.

Turk, D. C., Dworkin, R. H., Allen, R. R., et al. (2003). Core outcome domains for chronic pain clinical trials: IMMPACT recommendations. Pain, 106, 337-345.

Ubbink, D. T. H., Spincemaille, G. H. J. J., Prins, M. H., Reneman, R. S., \& Jacobs, M. J. H. M. (1999). Microcirculatory investigations to determine the effect of spinal cord stimulation for critical leg ischemia; The Dutch Multicenter Randomized Controlled Trial. Journal of Vascular Surgery, 30, 236-244. 



\section{Chapter 3}

\section{Long-term effects of spinal cord stimulation in patients}

\section{with painful diabetic neuropathy}

Cecile C de Vos ${ }^{1,2,3,4}$ Mathieu WPM Lenders ${ }^{1,3}$ Michel JAM van Putten ${ }^{2,4}$

1. Department of neurosurgery, Medisch Spectrum Twente, the Netherlands

2. Department of neurology and clinical neurophysiology, Medisch Spectrum Twente, the Netherlands

3. Stichting the neurobionics foundation, Enschede, the Netherlands

4. MIRA biomedical technology and technical medicine, CNPH group, University of Twente, the Netherlands

Submitted to Diabetic Medicine 


\section{ABSTRACT}

Aim: Diabetic neuropathy is a progressive disease and assessment of the persistence of pain relief by spinal cord stimulation is relevant before large numbers of patients with painful diabetic neuropathy are treated with spinal cord stimulation.

Methods: Nine patients with painful diabetic neuropathy and treated with spinal cord stimulation were initially followed for 2.5 years. Six patients from the original study cohort were interviewed 6.5 years post-implantation to assess long-term the effects of spinal cord stimulation on pain, medication intake, course of the disease and patient satisfaction.

Results: Average pain score on visual analogue scale was 77 at baseline and 23 twelve months post-implantation. After 6.5 years of spinal cord stimulation the remaining six patients had an average score for pain of 27 , two patients did still not use any analgesic medication and five patients had still significantly improved glycaemic control.

Conclusion: Spinal cord stimulation offers an effective therapy for chronic diabetic neuropathic pain and despite the progressive nature of diabetic neuropathy, the pain relief shows to be sustainable over the long term. 


\section{INTRODUCTION}

Despite adequate glycaemic control, up to $15 \%$ of the diabetic population develops painful peripheral neuropathic symptoms [1]. Painful diabetic neuropathy (PDN) generally starts in the toes and gradually progresses proximally. At a later stage, it may affect the hands as well, following the typical symmetrical 'glove and stocking' pattern. Although new drugs targeting neuropathic pain have become available, only about one third of the patients with PDN obtains over $50 \%$ pain relief through medication $[2,3]$. This motivates the search for alternative therapies to target PDN.

Spinal cord stimulation (SCS) is electrical stimulation of the dorsal columns of the spinal cord to interrupt or decrease neuropathic pain perception. A stimulation electrode lead is surgically implanted in the epidural space and connected subcutaneously to an implanted pulse generator. SCS has demonstrated to be effective as a therapy for different chronic painful conditions [4], but the effectiveness of this treatment for PDN is not well established yet. Only a few pilot studies have been published. In 1996, Kumar and co-workers [5] investigated the results of SCS in a group of patients with peripheral neuropathy, including four patients with PDN. All four diabetic patients reported good results on the short term (three months) and three on the long term (twelve months or over). Also in 1996, Tesfaye and co-workers [6] reported eight patients with PDN who received SCS therapy and demonstrated significant pain relief for at least one year in seven patients.

Since diabetic neuropathy is a progressive disease, assessment of both the persistence of pain relief and the effects of SCS on the course of the disease are of interest before large numbers of patients with PDN are treated with SCS. However, the number of diabetic patients treated with SCS and followed for a period of years is limited. Daousi and co-workers [7] showed continued pain relief in four patients up to seven years of stimulation. More recently, we performed a pilot study with SCS in eleven patients with PDN [8]. Statistically significant pain relief and reduction in analgesic medication was achieved up to 2.5 years post-implantation. In this communication, we present additional data from this cohort reporting the long-term effects of SCS. 


\section{METHODS}

Six patients with PDN from the original study cohort [8] who still had a stimulator participated and were interviewed. Effects of SCS on pain, medication intake and patient satisfaction after six, twelve and thirty months were compared to the effects after 6.5 years of stimulation.

Neuropathic pain relief was assessed by Visual Analogue Scale (VAS, from 0 indicating no pain to 100 worst possible pain); patients rated their overall pain four times a day for four consecutive days and supplied information about the analgesics they used. A structured interview was performed to assess satisfaction with SCS therapy. Patient global impressions of change (PGIC) on pain and on health were measured on a 7-point scale, from 1 representing very much improved to 7 very much worsened. Whether or not they would choose again for SCS therapy and recommend the therapy to other patients was measured on a 4-point scale. Surgeries and complications related to SCS and progression of PDN were registered.

\section{RESULTS}

Nine patients with PDN originally received a SCS system (table 1). Two patients died of cancer: one patient at 2 years and one patient at 5.5 years post-implantation. Due to insufficient effect of the stimulation, one patient had the stimulator explanted when the battery was empty 5 years post-implantation and refused participation. Six patients were interviewed 6.5 year post-implantation (range 6 - 7 years).

Table 1. Patient characteristics at baseline and results after 6.5 years of spinal cord stimulation.

\begin{tabular}{lrrrrrrrrr}
\hline patient & $\mathbf{1}$ & $\mathbf{2}$ & $\mathbf{3}$ & $\mathbf{4}$ & $\mathbf{5}$ & $\mathbf{6}$ & $\mathbf{7}^{\mathbf{a}}$ & $\mathbf{8}^{\mathbf{b}}$ & $\mathbf{9}^{\mathbf{c}}$ \\
\hline age (years) & 49 & 59 & 72 & 59 & 66 & 54 & 73 & 74 & 45 \\
diabetes type & $\mathrm{II}$ & $\mathrm{I}$ & $\mathrm{I}$ & $\mathrm{I}$ & $\mathrm{I}$ & $\mathrm{I}$ & $\mathrm{I}$ & II & I \\
diabetes (years) & 7 & 50 & 10 & 14 & 26 & 8 & 24 & 15 & 35 \\
pain (years) & 5 & 20 & 4 & 12 & 7 & 12 & 5 & 7 & 10 \\
VAS pain baseline & 80 & 85 & 60 & 90 & 80 & 80 & 80 & 75 & 65 \\
VAS pain 6.5 years & 30 & 20 & 20 & 25 & 25 & 40 & - & - & - \\
PGIC 0.5 years & 1 & 1 & 2 & 2 & 2 & 2 & 1 & 3 & 1 \\
PGIC health 6.5 years & 2 & 2 & 6 & 3 & 2 & 7 & - & - & - \\
PGIC pain 6.5 years & 2 & 1 & 2 & 2 & 2 & 6 & - & - & - \\
HbA1c baseline (\%) & 8.9 & 8.1 & 7.2 & 7.3 & 7.0 & 8.9 & 7.2 & 7.5 & 7.4 \\
HbA1c 6.5 years (\%) & 9.3 & 6.6 & 6.1 & 6.1 & 6.3 & 5.5 & - & - & - \\
\hline
\end{tabular}

VAS: visual analogue scale (0 no pain, 100 worst possible pain), PGIC: patient global impression of change ( 1 very much improved, 7 very much worsened), a: died 2 years post-implantation, b: died 5.5 years post-implantation, c: SCS system explanted after 5 years. 
Average VAS score for pain for all nine patients was 77 at baseline and 34 after six months of SCS. Both after twelve and thirty months of SCS, the average VAS score was 23 . After 6.5 years of SCS the remaining six patients had an average VAS score of 27 (figure 1).

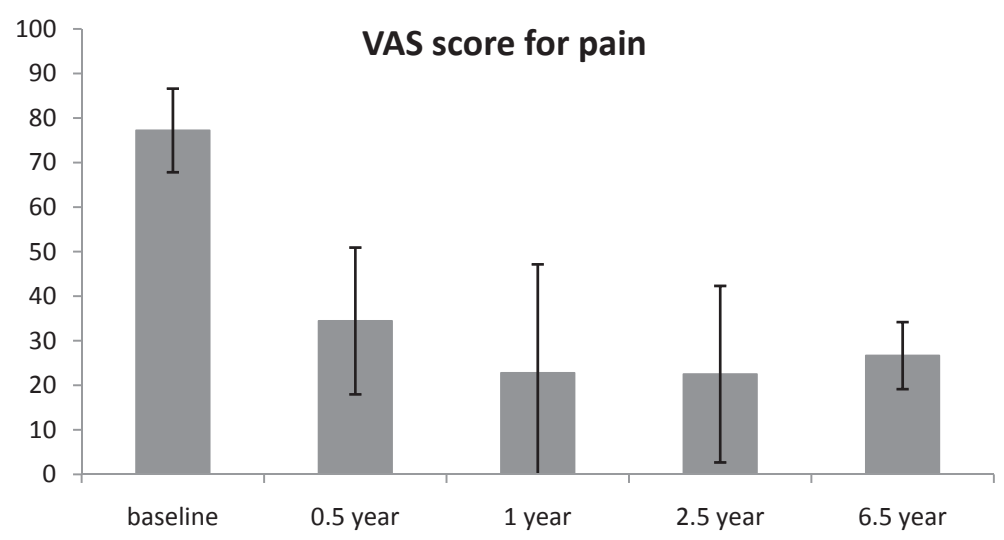

Figure 1. The pain scores on visual analogue scale (VAS, from 0 indicating no pain to 100 worst possible pain) for the patients of the original study cohort at baseline and after 6 months, 1 year $(n=9), 2.5$ years $(n=8)$ and 6.5 years $(n=6)$ of follow up. Bars represent the average pain scores, error bars represent the standard deviation.

Prior to implantation all patients used gabapentin or opioids, but these analgesics caused unacceptable side effects and provided insufficient pain relief. After twelve months of SCS the stimulator was the sole treatment for six patients and after thirty months three patients did still not use any analgesics. After 6.5 years, two patients did not use any analgesics, one patient used analgesic medication because of osteoarthritis and one patient because of complex regional pain syndrome. Two patients had to use analgesics because of PDN of the hands.

After six and thirty months of SCS therapy patients provided a PGIC score for their overall status and all patients indicated improvement at both time points. After 6.5 years of SCS the PGIC question was divided into separate questions for pain and health status. Compared to their health prior to implantation, two patients indicated a worsening, the other four indicated improvement. Five patients indicated that their pain was still improved, while one patient rated his pain as much worsened. Given the choice, all six patients would choose for SCS therapy again and all would recommend SCS therapy to other patients with PDN. 
During the first 2.5 years post-implantation, two surgical revisions of the SCS system and one battery replacement were necessary. During the next four years, six empty batteries had to be replaced and one system was explanted. Besides, two electrode leads had to be replaced: one because of increased impedance, probably due to scar tissue and one electrode migrated. Both percutaneous electrodes (Quad, Medtronic, MN, USA) were replaced by surgical, paddle-shaped electrodes (Tripole 16C, St Jude Medical, TX, USA).

Five years post-implantation one patient had all her toes amputated because of necrosis. Yet, after 6.5 years of SCS the area of her lower limbs affected by diabetic neuropathy had not increased compared to baseline. This was also the case in three other patients. Two patients however had proximally progression of the painful area in their lower limbs. In addition, diabetic neuropathic pain had evolved in the hands of two patients.

Two patients had a reduction in insulin requirement since implantation of the SCS system, the other patients did not show significant changes in insulin requirements compared to baseline. Five out of six patients mentioned an improvement in glycaemic control corresponding with decreased average $\mathrm{HbA} 1 \mathrm{c}$ levels, from $7.7 \%$ prior to implantation (61 $\mathrm{mmol} / \mathrm{mol}$ ) to $6.7 \%(50 \mathrm{mmol} / \mathrm{mol}) 6.5$ years post-implantation.

\section{DISCUSSION}

Effects of SCS therapy in patients with PDN have been scarcely studied $[5,7,8]$. Here, we presented data showing that long-term pain reduction can be achieved in PDN. Six patients participated in this follow-up study and demonstrated that a favourable initial response to SCS therapy is sustainable up to at least 6.5 years. All six patients still experienced pain reduction of $50 \%$ or more compared to baseline. However, four patients mentioned some increase in pain intensity when compared to 2.5 years post-implantation. These results are comparable to the results in four patients followed for seven years by Daousi et al. [7]. Two of these patients had still more than $50 \%$ pain reduction compared to baseline and all still experienced more than $50 \%$ pain reduction when comparing stimulation 'on' to stimulation 'off'. 
For six of our patients the stimulator was the sole treatment for their neuropathic pain one year post-implantation. Because of the side-effects of analgesics, reduction in analgesic intake was an important objective for all patients. However, after 6.5 years four patients had restarted analgesic intake, yet not because of neuropathic pain in their lower limbs, but because of new diseases or neuropathic pain in their hands.

Various effects of SCS besides pain relief that could be beneficial for patients with diabetes have been described. SCS was found to increase skin perfusion in the affected area and decrease amputations in diabetic patients with peripheral arterial occlusive disease [9]. An increase in perfusion might also slow down the progression of the affected body areas. In four patients the painful area of the legs had not changed; whether this can be contributed to SCS should be studied in a larger population. Fortunately, the enlarged painful area in the other two patients could be covered with new stimulation configurations on the originally implanted lead, since this lead was placed at thoracic level T10 and able to cover knees and upper legs as well. Treating neuropathic pain of the hands would require stimulation of the cervical spinal cord and hence implantation of a second electrode lead.

SCS was also found to significantly reduce insulin requirement in a patient with type II diabetes [10]. This case report described a $50 \%$ reduction of insulin up to two months after starting SCS treatment. None of our patients showed such a large decrease in insulin requirements after implantation. However, after 6.5 years of SCS two patients had still some reduction and the four other patients had no increase in insulin requirements as compared to baseline. Moreover, the average glycaemic control in five out of six patients had improved after starting SCS treatment and this effect was still present 6.5 years after implantation.

In conclusion, SCS offers an effective therapy for PDN and despite the progressive nature of diabetic neuropathy, pain relief is sustainable over the long term. However, effects of SCS on the course of diabetic neuropathy need to be studied in a larger group of patients. 


\section{REFERENCES}

1. Tesfaye, S., Vileikyte, L. Rayman, G. et al. (2011). Painful diabetic peripheral neuropathy: consensus recommendations on diagnosis, assessment and management. Diabetes metab res rev 27: 629-638.

2. Jensen, T. S., Backonja, M.-M., Hernández Jiménez, S., Tesfaye, S., Valensi, P., \& Ziegler, D. (2006). New perspectives on the management of diabetic peripheral neuropathic pain. Diabetes \& vascular disease research: official journal of the International Society of Diabetes and Vascular Disease, 3:108-119.

3. Spallone, V. (2012). Management of painful diabetic neuropathy: guideline guidance or jungle? Current diabetes reports, 12:403-413.

4. Mailis-Gagnon, A. Furlan, A.D., Sandoval, J. A. \& Taylor, R.S. (2004). Spinal cord stimulation for chronic pain. Cochrane Database of Systematic Reviews 2004, Issue 3. Art.No.: CD003783.

5. Kumar, K., Toth, C., \& Nath, R. (1996). Spinal cord stimulation for chronic pain in peripheral neuropathy. Surgical neurology, 3019:363-369.

6. Tesfaye, S., Watt, J., Benbow, S. J., Pang, K. A, Miles, J., \& MacFarlane, I. A. (1996). Electrical spinal-cord stimulation for painful diabetic peripheral neuropathy. Lancet, 348:1698-701.

7. Daousi, C., Benbow, S. J., \& MacFarlane, I. a. (2005). Electrical spinal cord stimulation in the long-term treatment of chronic painful diabetic neuropathy. Diabetic medicine: a journal of the British Diabetic Association, 22: 393-398.

8. De Vos, C. C., Rajan, V., Steenbergen, W., Van der Aa, H. E., \& Buschman, H. P. J. (2009). Effect and safety of spinal cord stimulation for treatment of chronic pain caused by diabetic neuropathy. Journal of diabetes and its complications, 23: 40-45.

9. Petrakis, I. E., \& Sciacca, V. (2000). Epidural spinal cord electrical stimulation in diabetic critical lower limb ischemia. Journal of diabetes and its complications, 13:293-9.

10. Kapural, L., Hayek, S. M., Stanton-Hicks, M., \& Mekhail, N. (2004). Decreased Insulin Requirements with Spinal Cord Stimulation in a Patient with Diabetes. Anesthesia \& Analgesia, 745-746. 


\section{Chapter 4}

\section{Spinal cord stimulation in patients with painful diabetic}

\section{neuropathy: a multi centre randomised clinical trial}

CC de Vos ${ }^{1,2}$, K Meier $^{3}$, P Brocades Zaalberg ${ }^{4}$, HJA Nijhuis ${ }^{5}$, W Duyvendak ${ }^{6}$, J Vesper ${ }^{7}$, TP Enggaard ${ }^{8}$, MWPM Lenders ${ }^{1}$

${ }^{1}$ Medisch Spectrum Twente, Enschede, the Netherlands

${ }^{2}$ Department of Clinical Neurophysiology, University of Twente, The Netherlands

${ }^{3}$ Aarhus University Hospital, Aarhus, Denmark

${ }^{4}$ St Jansdal Ziekenhuis, Harderwijk, the Netherlands

${ }^{5}$ St Antonius Ziekenhuis, Nieuwegein, the Netherlands

${ }^{6}$ Virga Jesse Hospital, Hasselt, Belgium

${ }^{7}$ Universitätsklinikum, Düsseldorf, Germany

${ }^{8}$ Odense University Hospital, Odense, Denmark 


\section{ABSTRACT}

Objective: The pathophysiology of painful diabetic neuropathy is complex and multifactorial, and the pain is often very difficult to treat. Spinal Cord Stimulation (SCS) has proven to be an effective therapy for various types of chronic neuropathic pain, yet effectiveness of SCS treatment for painful diabetic neuropathy (PDN) is not well established. This European multi-centre randomized controlled trial studies the effectiveness of SCS in patients with PDN.

Method: Sixty patients with PDN in the lower extremities, refractory to conventional medical therapy, were enrolled and followed for six months. Patients were randomized 2:1 to best conventional medical practice with (SCS group) or without (control group) additional SCS therapy, and both groups were followed up at regular intervals. At each follow-up, pain intensity (VAS, 0-100) and analgesics consumption were recorded along with completion of the EuroQoL 5D form (EQ5D) and the McGill pain questionnaire (MPQ). After six months of treatment all patients were given the opportunity to cross over to the other treatment group in case their assigned study treatment was not effective.

Results: The average VAS score at baseline was 73 for the SCS group and 67 for the control group. After six months of treatment the average VAS score was significantly reduced to 29 for the SCS group $(p<0.001)$ and remained 66 for the control group. In the SCS group, outcome of EQ5D and MPQ questionnaires improved significantly, while there were no significant changes in the control group.

Conclusion: Spinal Cord Stimulation caused significant pain reduction and improvement of quality of life in patients with refractory painful diabetic neuropathy in the lower extremities. 


\section{INTRODUCTION}

Diabetes mellitus is a chronic disease characterised by chronic hyperglycaemia and defects in insulin secretion, insulin action, or both. Diabetes can result in peripheral polyneuropathy in up to $50 \%$ of patients [Tesfaye 2011]. Despite adequate glycaemic control, up to $15 \%$ of the diabetic population develops painful peripheral neuropathic symptoms, mainly affecting the lower limbs [Tesfaye 2011; Spallone 2012; McGreevy 2012]. Although new drugs targeting neuropathic pain have become available over the last decades, only about one third of the patients with painful diabetic neuropathy (PDN) obtains over $50 \%$ pain relief through medication [Jensen 2006]. This motivates the need for alternative therapies to target PDN.

Spinal cord stimulation (SCS) is an invasive treatment for chronic pain based on electrical stimulation of the dorsal column of the spinal cord. SCS is believed to interrupt or reduce the pain signals sent from the painful body part towards the brain. Generally implantation of the SCS device comprises two phases. First, the electrode lead is implanted in the epidural space and connected to a temporary pulse generator outside the body. Only if this trial period is successful, that is, if a patient experiences significant pain relief, the external pulse generator will be converted into an implanted pulse generator.

SCS has shown to be an effective treatment for various neuropathic pain conditions [MailisGagnon 2004]. Although SCS is increasingly accepted in the treatment of failed back surgery syndrome (FBSS) [North 2005; Kumar 2007] and complex regional pain syndrome I (CRPS I) [Kemler 2000] and angina pectoris [De Jongste 1994; Mannheimer 1998], evidence of the effectiveness of SCS treatment in painful diabetic neuropathy is sparse; thus far no randomized controlled trials have been performed.

A number of studies have concentrated on the effects of SCS in patients with painful diabetic neuropathy, none of them were randomized; yet they have yielded encouraging results [Kumar 1996; Tesfaye 1996; Daousi 2005; De Vos 2009; Pluijms 2012]. The study carried out by Tesfaye and co-workers in ten patients suffering from PDN demonstrated satisfactory relief of pain during trial stimulation in eight patients and significant pain relief for at least one year in seven patients [Tesfaye 1996]. Long term follow-up of four of these patients was done by Daousi and co-workers and showed continued, pain relief after seven years of stimulation [Daousi 2005]. Kumar and co-workers similarly investigated the long term 
results of SCS treatment in a group of thirty patients with peripheral neuropathy [Kumar 1996]. Of these thirty patients four had peripheral neuropathy due to diabetes. All four diabetic pain patients reported good to very good results on the short term (3 months) and three out of four on the long term (12 months or over).

More recently, De Vos and co-workers carried out an SCS study in eleven patients suffering from painful diabetic neuropathy not responding to conventional treatment [De Vos 2009]. Nine subjects experienced satisfactory pain relief during trial stimulation and were converted to a permanent system. Pain intensity and analgesic medication were reduced significantly up to thirty months after implantation. Similar results were found in a pilot study by Pluijms and co-workers [Pluijms 2012]. Eleven out of fifteen patients experienced pain reduction during trial stimulation and ten patients continued to experience improvement on pain and quality of life after twelve months of SCS therapy.

In order to thoroughly investigate the effect of SCS in PDN we performed a prospective, multi centre, randomised clinical study comparing the efficacy of SCS therapy to best conventional medical practice.

\section{METHODS}

The SCS-001 study had an open, no placebo, parallel-group design with randomisation in a 2:1 ratio to the treatment or the control group. Patients were recruited in seven pain clinics in the Netherlands, Denmark, Belgium and Germany. The study conformed to the Declaration of Helsinki, was approved by the institutional review board of Medisch Spectrum Twente and was registered at the Dutch Trial Register (ISRCTN03269533). The institutional review board or ethics committee at each clinic approved the protocol as well and all patients gave informed consent prior to participation.

\section{Patients}

Sixty patients were included and, stratified for sex, randomly assigned to best conventional medical practice with (SCS group) or without (control group) additional SCS therapy. 
Eligible patients were 18 years of age, suffered from refractory diabetic neuropathic pain in the lower extremities that existed for more than one year and could not be treated further according to the referring medical specialist. In addition, patients should have an average pain score on a visual analogue scale (VAS) of at least 50 and should not have psychological problems that require treatment. Patients were excluded from participation in the study if they had an infection, had neuropathic pain in the upper extremities, used anti-coagulant medication or had coagulation irregularities, had a life expectancy of less than 1 year, were addicted or incapable of cooperation.

\section{Study procedure}

Patients were evaluated for eligibility during a 4-week screening period. After the baseline period, during which information on pain and overall health parameters was recorded, patients were randomised to either the SCS group or the control group. For all patients adjustment of medication and other conventional pain treatments were allowed. Patients in the SCS group received additional trial stimulation and were converted to a permanent SCS system if they experienced clinically relevant pain reduction during trial stimulation.

Implantation of the SCS system was performed according to each pain clinic's practice, but procedural and equipment variations were minimized. Antibiotic prophylaxis was administered and a trial stimulation period of seven days maximum was allowed to test whether a patient responded positively to SCS. A SCS electrode (Octrode or S8 lamitrode, St Jude Medical, Plano, TX, USA) was implanted in the epidural space until the patient reported optimal overlap between paresthesia and the painful area. The electrode lead was anchored to the fascia and connected through an extension to an external pulse generator (Multiprogram Trial Stimulator, St Jude Medical, Plano, TX, USA). If the trial period was successful, a pulse generator (Eon C, Eon or Eon Mini, St Jude Medical, Plano, TX, USA) was implanted subcutaneously in either the anterior abdominal wall or the upper buttock and connected to the electrode lead.

All patients had evaluation visits at 1, 3 and 6 months after initiation of SCS treatment (SCS group) or enrolment (control group). After six months patients in the control group, who have not had adequate improvement, could cross over to SCS therapy. After completion of the study period, all patients were followed in accordance with best standard medical care and evaluated for alternative therapies in case their assigned study treatments were not effective. 


\section{Outcome parameters}

In order to evaluate the efficacy over time of the addition of SCS treatment to conventional medical practice, pain scores and other health outcome parameters were acquired at all study visits.

The study's primary outcome parameters were the average pain reduction and the percentage of patients with over $50 \%$ pain reduction after six months of treatment in each study group. Additional outcome parameters were pain characteristics assessed by McGill Pain Questionnaire (MPQ), quality of life assessed by MPQ and EuroQoL 5D (EQ5D), medication intake and patient global impression of change (PGIC) were also measured and used to compare the efficacy of SCS treatment to conventional medical practice.

Pain scores were assessed using a visual analogue scale (VAS: from 0 representing no pain to 100 worst possible pain), the total number of words chosen from the McGill pain questionnaire (NWC) and the total pain rating index of these words (PRI) [Melzack 1987]. Health related quality of life was evaluated using the self-reported perception of health from the EQ5D questionnaire and questions about quality of life that are part of the standard Dutch language MPQ questionnaire (MPQ-DLV) [Verkes 1989].

The use of various types of analgesic medication was recorded and the medication quantification scale III (MQS) [Masters Steedman 1992; Harden 2005] was used to evaluate the intake of analgesics. The MQS score for a single medication is calculated by multiplying a score for the dosage used by the detriment weight for its given pharmacological class. The total MQS score is the sum of the calculated values for each medication.

After six months, patients were also asked to indicate on a four-point scale whether or not they would recommend the treatment they have had to other patients with PDN and to indicate their overall health and pain status on a seven-point patient's global impression of change (PGIC) scale [Farrar 2001]. The safety and tolerability of SCS therapy over time was evaluated using information on treatment emergent adverse events, device complications and premature withdrawal from the trial. 


\section{Statistical analysis}

Based on the results from the study by De Vos et al. [De Vos 2009] the percentage of patients with over $50 \%$ pain reduction in the SCS therapy group was assumed to be $50 \%$ versus $10 \%$ of the patients in the control group. The sample size calculated on these estimated response rates, 60 patients randomised 2:1 (40 with SCS therapy and 20 without SCS therapy), would provide a $90 \%$ power to find a difference between the two groups at a $5 \%$ level of significance.

Analysis of covariance (ANCOVA) was used to test for outcome differences between the SCS therapy group and the control group after six months of treatment. Between-group differences in continuous variables over time were assessed by analysis of repeated measurements (performed with SPSS procedure for mixed models, version 15). Baseline values were subtracted from follow-up values to correct for baseline differences. Additional analyses were performed using Fishers exact statistics for categorical variables.

Table 1. Patient characteristics at baseline.

\begin{tabular}{llll}
\hline & & SCS group & Control group \\
\hline Sex & female/male & $15 / 25$ & $7 / 13$ \\
Diabetes & type I/type II & $10 / 30$ & $5 / 15$ \\
Age & years (SD) & $58(11)$ & $61(12)$ \\
Duration diabetes & years & $16(11)$ & $17(12)$ \\
Duration pain & years & $7(6)$ & $7(6)$ \\
Pain & VAS & $73(16)$ & $67(18)$ \\
& NWC-T & $13(5)$ & $13(3)$ \\
Quality of Life & PRI-T & $27(13)$ & $24(9)$ \\
Self-reported health & MPQ score & $16(5)$ & $15(6)$ \\
Pain medication & VAS & $50(19)$ & $47(17)$ \\
\hline
\end{tabular}

\section{RESULTS}

\section{Patients}

Sixty patients with painful diabetic neuropathy were included (see table 1.). Baseline characteristics were relatively well balanced between the two groups, the main exceptions being a somewhat higher age and lower pain score in the control group, however both not statistically significant. 
Forty patients (15 female, 25 male) were randomised to the SCS treatment group. Their average age at enrolment was 58 years (range 36 - 78 years) and they had suffered from diabetes mellitus for 15 years and diabetic neuropathic pain for 7 years on average. Three patients did not experience satisfactory pain reduction during trial stimulation and were not converted to a permanent system. None of these patients wanted to continue to participate in the study, consequently no follow up data of these patients was available for analysis. Thirty-seven patients received a permanent SCS system, one patient dropped out after three months of SCS therapy, the others were followed for six months (see figure 1).

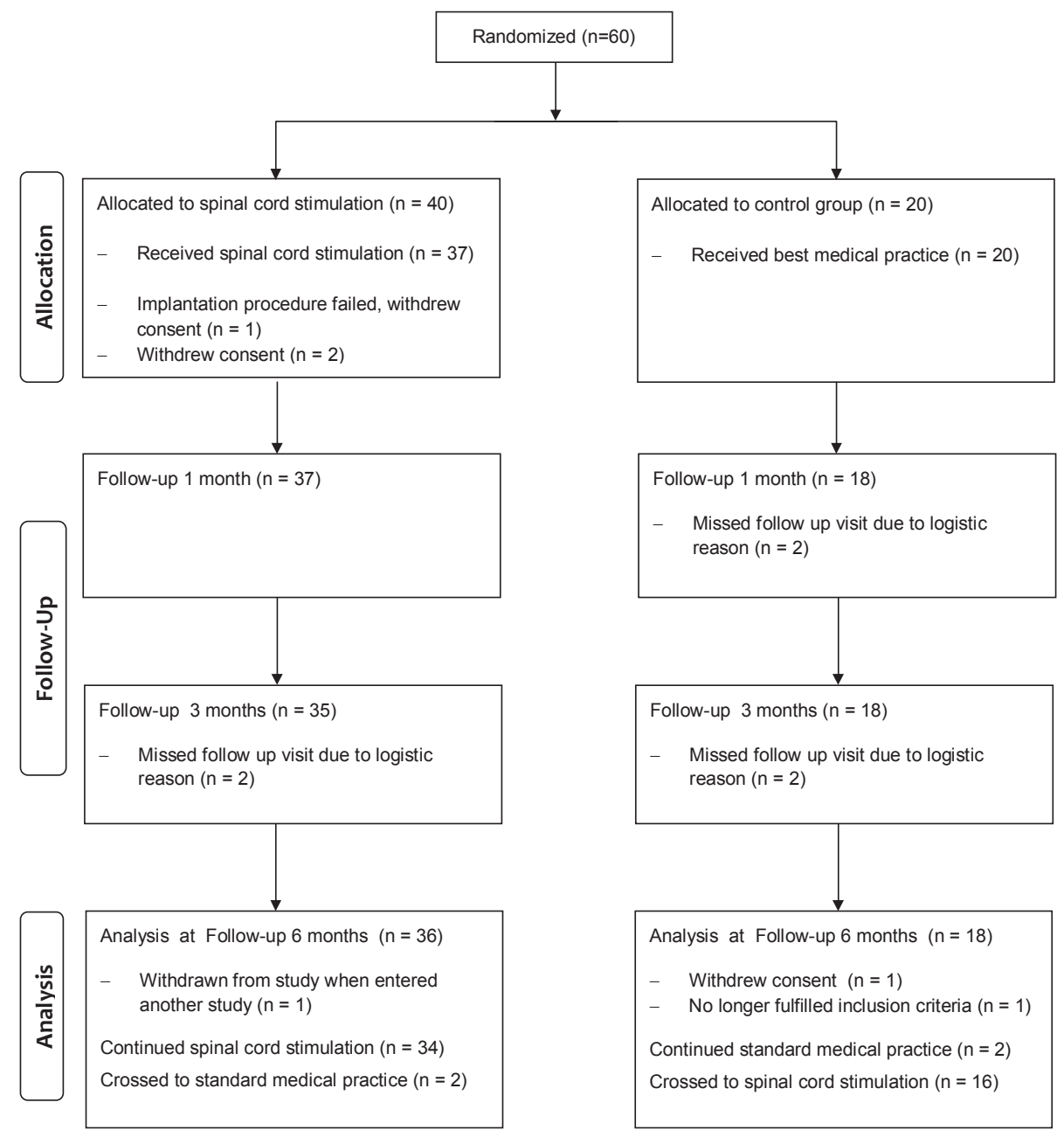

Figure 1. Flow chart of the study; 40 patients were randomized to the SCS group and 20 to the control group. At six months, 36 and 18 respectively remained in each group. 
Twenty patients ( 7 female, 13 male) were randomized to the control group. Their average age at enrolment was 61 years (SD 10 years) and they had suffered from diabetes mellitus for 17 years (SD 12 years) and diabetic neuropathic pain for 7 years (SD 6 years) on average. Two patients dropped out after 3 months of conventional pain treatment. Eighteen patients receiving conventional pain treatment were followed for six months. Subsequently, sixteen patients wanted trial stimulation and all of them were converted to a permanent system.

\section{Primary outcome measure}

Average pain score at enrolment was 73 (SD 16) for the SCS treatment group and 67 (SD 18) for the control group. After one month of SCS, the average pain score on VAS for the SCS group was already reduced to 27 (SD 26) and this VAS score demonstrated to be stable for the remaining study period (see figure 2 ).

For the SCS group, the average pain score on VAS after six months was 29 (SD 27). In this group, 24 patients had over $50 \%$ pain reduction, of whom 16 had a reduction of more than $75 \%$. Only one patient from the control group had over $50 \%$ pain reduction and despite medical treatment, the average pain score of this group maintained 66 (SD 22). Following six months of conventional medical practice, 16 out of the 18 remaining patients in the control group wanted trial stimulation and all were converted to a permanent SCS system.

\section{VAS score for pain}

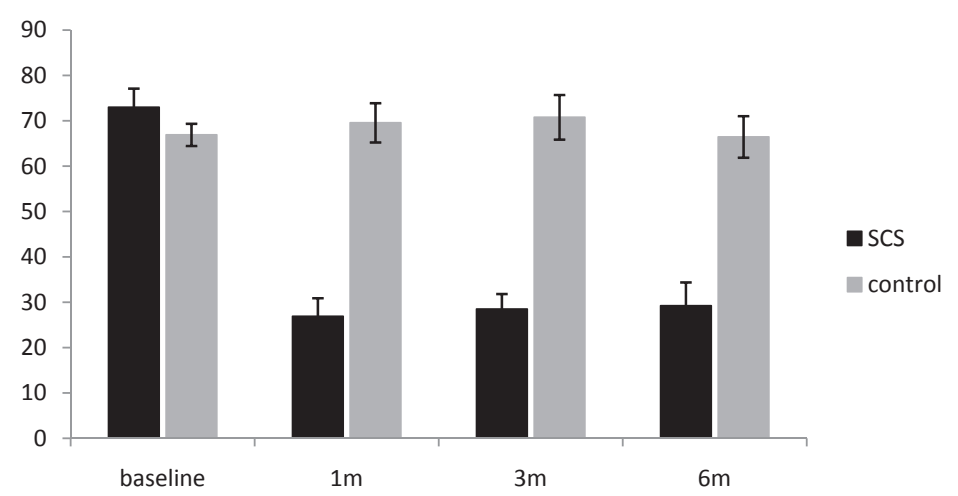

Figure 2. Average pain scores of the patients in the SCS treatment group and the control group at baseline, and after 1, 3 and 6 months of treatment. Error bars represent standard error. 


\section{Secondary outcome measures}

\section{Pain quality (MPQ)}

At baseline, patients in the SCS group used 13 (SD 5) words on average from the McGill pain questionnaire to describe their pain, with a total pain rating index (PRI) of 27 (SD 13). Patients in the control group used 13 (SD 3) words on average with PRI 24 (SD 9) (figure 3). After six months, the average number of words used dropped to 8 (SD 6) with PRI 15 (SD 14) in the SCS group, while in the control group the total number of words remained at 13 (SD 4) with PRI 26 (SD 10).

A

NWC

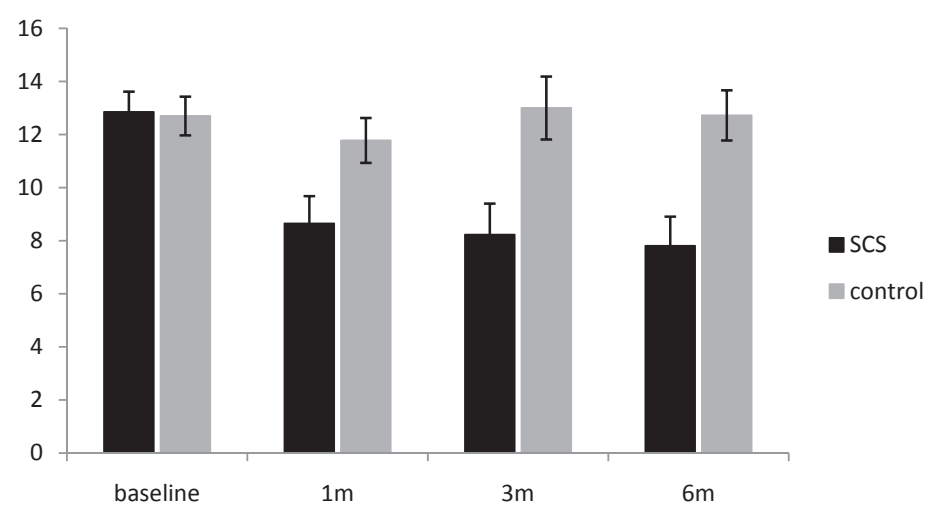

B

PRI

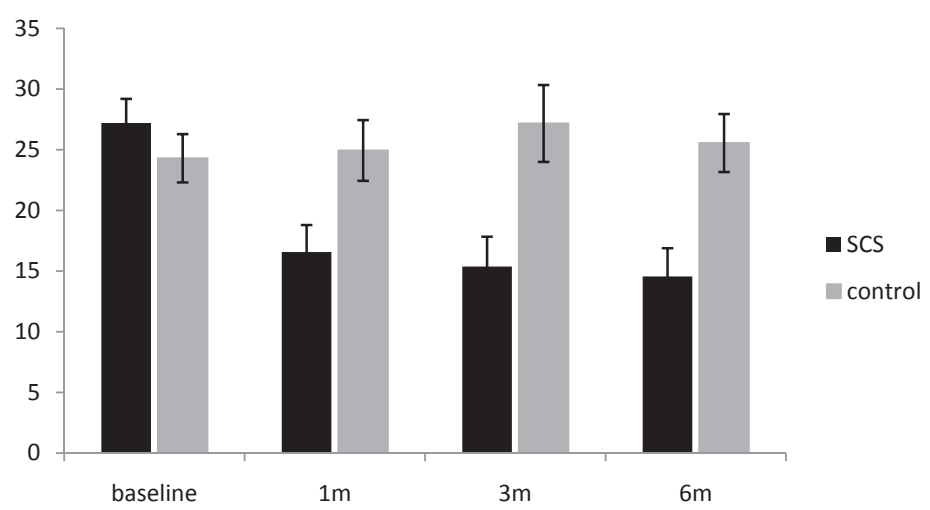

Figure 3. (a) Average total number of words chosen from the McGill pain questionnaire to describe the perceived pain and (b) the average total pain rating index of these words for both the SCS treatment group and the control group. 


\section{Quality of Life (MPQ)}

The McGill pain questionnaire Quality of Life score (MPQ-QoL) [Verkes 1989] is higher, the more pain patients feel or the more they are disturbed by pain in their daily activities or sleep. At baseline the average MPQ-QoL score was 16 (SD 5) in the SCS group and 15 (SD 6) in the control group (figure 4). In both groups $70 \%$ of the patients had disturbed sleep and $90 \%$ had to rest several hours a day because of their pain.

After six months of SCS therapy for the SCS group, the average MPQ-QoL score improved to 7 (SD 7) and only 11 patients (28\%) had problems sleeping. The control group maintained an average MPQ-QoL score of 14 (SD 6) and 12 patients (67\%) had disturbed sleep.

\section{MPQ QOL}

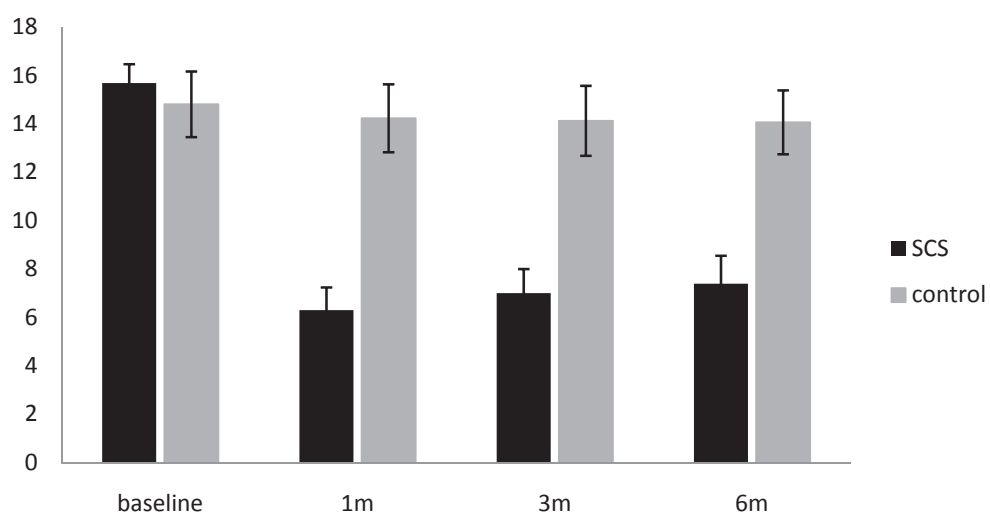

Figure 4. Average McGill pain questionnaire Quality of Life scores for both the SCS treatment group and the control group. A high score corresponds with severe pain and disturbed daily activities. Error bars represent standard error.

\section{Self-reported health (EuroQoL 5D)}

The EuroQoL 5D records a.o. the respondent's self-rated health on a visual analogue scale on which 100 corresponds with the best imaginable health state and 0 with the worst imaginable health state. At baseline, patients in the SCS group rated their health at 50 (SD 19) on average and patients in the control group rated their health at 47 (SD 17). The SCS group indicated score of 61 (SD 23) after six months of SCS therapy. In contrast, patients in the control group indicated a score of 41 (SD 20) after six months (figure 5). 


\section{self-reported health}

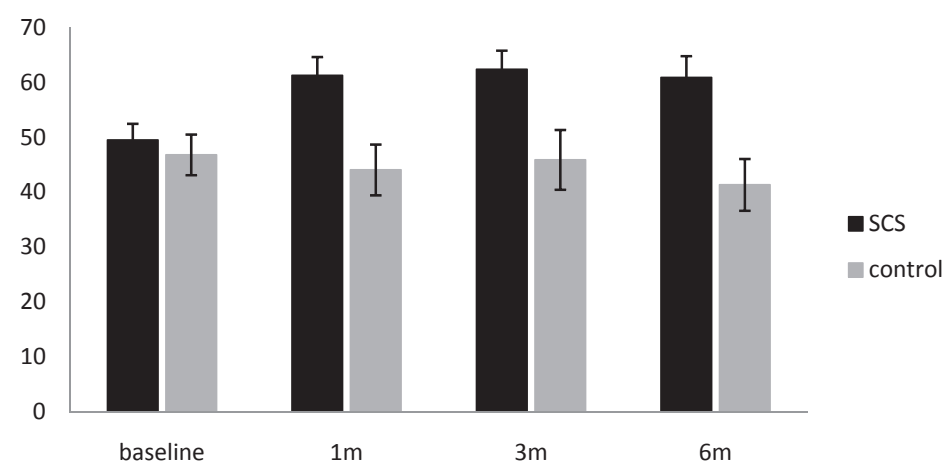

Figure 5. Average self reported health measured on a visual analogue scale on the EQD5 questionnaire; 100 indicates the best and 0 the worst imaginable health state. Error bars represent standard error.

\section{Medication}

At baseline $70 \%$ of the patients of both groups used anticonvulsant medication (carbamazepine, gabapentin, pregabalin) or antidepressants (amitriptyline, duloxetine, imipramine, nortriptyline), $45 \%$ of the patients used opioids like oxycodon, fentanyl, morphine and methadone, $20 \%$ of the patients used non-steroid anti-inflammatory drugs (diclofenac, meloxicam) and 30\% of the patients used acetaminophen. A large majority of patients used combinations of various analgesics. Five patients from the SCS group and three patients from the control group had chosen not to use any prescribed pain medication anymore due to unacceptable side effects combined with only limited effects.

The average medication quantification scale (MQS) for the analgesics at baseline used by the patients in the SCS group was 10.6 (SD 9.7) and was 9.2 (SD 7.8) for the patients in the control group. The average MQS of the SCS group was reduced to 7.5 (SD 8.6) after six months of SCS therapy. At that time, about $45 \%$ of these patients used neurotropic medication, 35\% used opioids and eight patients did not use any pain medication anymore.

After six months of conventional pain treatment, the average MQS of the control group had not significantly changed and remained 9.8 (SD 8.3); still $60 \%$ of these patients used neurotropic medication, $50 \%$ used opioids and one patient did not use pain medication. 


\section{Patient satisfaction}

The patients' global impression of change (PGIC) was assessed after six months of treatment for both groups. In the SCS group 25 patients stated that they had much or very much pain reduction and three patients indicated some pain reduction, while only three patients from the control group indicated some pain reduction and despite conventional pain treatment eight patients even perceived an increase in pain. In the SCS group all but two patients would recommend SCS treatment to other patients with diabetic neuropathic pain. In the control group 4 patients might recommend conventional pain treatment to other patients. Patients in the SCS group were content with the treatment they received and graded the SCS treatment on average 8/10; patients in the control group graded conventional pain treatment 4/10.

\section{Drop outs}

In the SCS group, three patients did not have successful trial stimulation and were therefore not converted to a permanent SCS system. Two patients did not experience satisfactory pain reduction during trial stimulation and in one patient it was impossible to position an electrode lead. One patient was withdrawn from the study after three months of SCS, when he entered another study. After six months of SCS treatment, two patient experienced insufficient pain reduction and had their stimulators explanted.

In the control group, two patients dropped out after three months of conventional pain treatment, unwilling to continue participation because of new diseases.

After six months of conventional medical treatment, sixteen from the remaining eighteen patients wanted trial spinal cord stimulation because of insufficient pain relief. Despite insufficient pain reduction, one patient did not want to switch to SCS therapy because of other health problems. One patient obtained significant pain relief because of adjusted pain medication and therefore did not need to switch to SCS therapy.

There is no data available from the patients after their drop out, as none of them was willing to continue to participate in the study. 


\section{Adverse events}

In both groups, adverse events that were not related to the study procedure occurred both before and after implantation of the SCS system. Serious adverse events prior to implantation in the SCS group were a femur fracture and an infection causing unstable blood glucose levels. After implantation, one patient got trochanteric bursitis, one patient had long-lasting unstable blood glucose levels and one patient had a cardiac arrest.

In the control group, two patients had infections, one patient got stenosis of the carotid and a myocardial infarction, one patient got angina pectoris and had coronary bypass surgery and one patient got atrial fibrillation.

The following serious adverse events were related to or interfered with the study procedure: two pulse generators had to be repositioned because one pulse generator had tilted and one pulse generator caused pain, three leads had to be repositioned because of lead migration or incomplete overlap of the paresthesia with the painful area, one patient turned out to have a coagulopathy which complicated the implantation procedure, and one patient had an infection of the lead during trial stimulation.

\section{DISCUSSION}

In patients with refractory painful diabetic neuropathy SCS therapy caused an average pain reduction of $65 \%$ where on average conventional pain treatment was not able to cause any pain reduction. The pain relief due to spinal cord stimulation was already apparent after one month and the effect demonstrated to be stable over the next six months. The percentage of patients with over $50 \%$ pain reduction after six months of SCS was $65 \%$, while only one patient (6\%) in the control group perceived over $50 \%$ pain reduction. In this randomized controlled trial we have demonstrated that the results of SCS therapy for patients with painful diabetic neuropathy are comparable or compare favourably to the results in patients with CRPS and FBSS.

In the RCT with CPRS patients performed by Kemler and co-workers, physical therapy with adjuvant SCS therapy is compared to physical therapy alone [Kemler 2000]. In the CRPS study 24 out of 36 patients with CRPS had successful trial stimulation, were converted to a permanent system and had an average reduction of 36 VAS points after six months of SCS therapy. In patients with painful diabetic neuropathy 37 out of 40 patients had successful 
trial stimulation and had an average pain reduction of 44 VAS points after six months of stimulation.

The RCT with FBSS patients, comparing SCS therapy to reoperation, performed by North and co-workers [North 2005] showed that 19 out of 24 patients had a successful trial stimulation and were converted to a permanent system. After six months 9 out of 19 (47\%) patients with SCS therapy had over $50 \%$ pain reduction.

The consecutive PROCESS study is a large RCT that randomized 100 FBSS patients to either SCS therapy or conventional medical treatment [Kumar 2007]. The results of the PROCESS study are comparable with the preceding RCT in FBSS, as 24 out of 48 patients (50\%) with a stimulator achieving over $50 \%$ pain reduction after six months of SCS therapy. Five out the 48 patients (10\%) wished to stop SCS therapy after six months of therapy, while 32 out of 44 (73\%) patients in de control group wished to switch to SCS therapy.

At enrolment the control group had a slightly lower pain score, a slightly lower health score and used somewhat less medication. However, none of the differences between the two groups at baseline was statistically significant.

A possible limitation of this study is that conventional pain treatment is not perceived as proper treatment by some of the patients in the control group. These patients generally already tried most, or all, of the conventional pain treatment options, including various types of pain medication, physical therapy, transcutaneous electrical nerve stimulation (TENS) and coping programmes, which limited the possibilities for further treatment options for the control group.

Besides, the control group knew that after six months of conventional medical treatment they would be offered trial spinal cord stimulation. It cannot be ruled out that some of the data collected in this group was biased by this prospect. However, the decrease in selfreported health during the six months of follow up was not statistically significant.

The pain reduction patients obtained after one month of SCS therapy was significant and maintained at the same level during the six months of follow up. It is therefore unlikely that the pain reduction is solely a placebo effect, in particular because diabetic neuropathy is a disease that worsens over time. Though, this could only be certified in a placebo controlled 
trial. However, at the time we designed this study, a placebo controlled protocol was not feasible, as new paresthesia-free stimulation paradigms [De Ridder 2013, Van Buyten 2012] were not yet available.

In addition to pain reduction and improvement in quality of life, SCS therapy demonstrated to lead to a reduction in pain medication intake. Although the reduction in analgesics is statistically significant, it is not as large as it was in the pilot study, where more than $50 \%$ of the patients could stop with the intake of analgesics [De Vos 2009]. The patients in the current study were not only suffering from painful neuropathy, but also from other diseases whether or not caused by diabetes mellitus. Probably these patients had more health related problems than the patients in the pilot study, which limited the possibilities for medication reduction.

Patients who have diabetes mellitus and unstable blood glucose levels for over 15 years usually have several other health related problems besides the neuropathy. Most of the serious adverse events were related to the vulnerable condition of the patients and preexisting health problems.

Patients with diabetes mellitus are susceptible for infections, so a short trial period is prudent. On the other hand, due to disturbed sensory perception it can take hours before a patient will feel changes in sensation caused by stimulation of the spinal cord. Patience and careful consideration regarding lead positioning and duration of the trial period are necessary. According to a review on SCS by Compton et al. [2012], infections occur in up to $8 \%$ of the patients while lead complications happen in $10-30 \%$ of the patients. The incidences in this study were 3\% (1 patient) infection of the lead and $8 \%$ (3 patients) lead complications.

Compared with conventional pain treatment, spinal cord stimulation causes significant pain reduction and improvement of quality of life in patients with refractory painful diabetic neuropathy in the lower extremities.

\section{Acknowledgements}

This study was sponsored by St Jude Medical. The authors would like to thank Andre Elands and Koert Hermans for their support throughout the study. 


\section{REFERENCES}

Compton AK, Shah B, Hayek SM. Spinal Cord Stimulation: A Review. Curr Pain Headache Rep 2012; 16: 35-42

Daousi C, Benbow SJ, MacFarlane IA. Electrical spinal cord stimulation in the long-term treatment of chronic painful diabetic neuropathy. Diabet Med 2005; 22: 393-398

de Jongste MJ, Hautvast RW, Hillege HL, Lie KI. Efficacy of spinal cord stimulation as adjuvant therapy for intractable angina pectoris: a prospective, randomized clinical study. Working Group on Neurocardiology. J Am Coll Cardiol 1994; 23: 1592-1597

De Ridder D, Plazier M, Kamerling N, Menovsky T, Vanneste S. Burst Spinal Cord Stimulation for Limb and Back Pain. World Neurosurg 2013. doi: 10.1016/j.wneu.2013.01.040

De Vos CC, Rajan V, Steenbergen W, Van der Aa HE, Buschman HPJ. Effect and safety of spinal cord stimulation for treatment of chronic pain caused by diabetic neuropathy. Journal of diabetes and its complications 2009; 23: $40-45$

Farrar JT, Young Jr JP, LaMoreaux L, Werth JL, Poole RM. Clinical importance of changes in chronic pain intensity measured on an 11-point numerical pain rating scale. Pain 2001; 94: 149-158

Harden RN, Weinland SR, Remble TA, Houle TT, Colio S, Steedman S, Kee WG. Medication Quantification Scale Version III: Update in Medication Classes and Revised Detriment Weights by Survey of American Pain Society Physicians, The Journal of Pain 2005; 6: 364-371

Jensen TS, Backonja M, Hernández Jiménez S, Tesfaye S, Valensi P, Ziegler D. New perspectives on the management of diabetic peripheral neuropathic pain. Diabetes Vasc Dis Res 2006;3:108-119

Kemler MA, Barendse GAM, van Kleef M, de Vet HCW, Rijks CPM, Furnee CA, van den Wildenberg FAJM. Spinal cord stimulation in patients with chronic reflex sympathetic dystrophy. N Engl J Med 2000; 343: 618-24

Kumar K, Toth C, Nath R. Spinal cord stimulation for chronic pain in peripheral neuropathy. Surgical neurology 1996; 3019: 363-369

Kumar K, Taylor RS, Jacques L et al. Spinal cord stimulation versus conventional medical management for neuropathic pain: a multicentre randomised controlled trial in patients with failed back surgery syndrome. Pain 2007; 132: 179-188

Mailis-Gagnon A, Furlan AD, Sandoval JA, Taylor RS. Spinal cord stimulation for chronic pain. Cochrane Database of Systematic Reviews 2004, Issue 3. Art. No.: CD003783. DOI: 10.1002/14651858.CD003783.pub2. Mannheimer 1998

Mannheimer C, Eliasson T, Augustinsson LE et al. Electrical stimulation versus coronary artery bypass surgery in severe angina pectoris: the ESBY study. Circulation 1998; 97: 1157-1163

Masters Steedman S, Middaugh SJ, Kee WG, Carson DS, Harden RN, Miller MC. Chronic-pain medications: equivalence levels and method of quantifying usage. Clin J Pain 1992; 8: 204-214

McGreevy K, Williams KA. Contemporary Insights into Painful Diabetic Neuropathy and Treatment with Spinal Cord Stimulation. Curr Pain Headache Rep 2012; 16: 43-49

Melzack R. The short-form McGill Pain Questionnaire. Pain 1987; 30: 191-197

North RB, Kidd DH, Farrokhi F, Piantadosi SA. Spinal cord stimulation versus repeated lumbosacral spine surgery for chronic pain: a randomized, controlled trial. Neurosurgery 2005; 56: 98-106

Pluijms WA, Slangen R, Bakkers M, Faber CG, Merkies ISJ, Kessels AG, Dirksen CD, Joosten EA, Reulen JPH, van Dongen RT, Schaper NC, van Kleef M. Pain relief and quality-of-life improvement after spinal cord stimulation in painful diabetic polyneuropathy: a pilot study. British Journal of Anaesthesia 2012. doi:10.1093/bja/ aes 251

Spallone V. Management of painful diabetic neuropathy: guideline guidance or jungle? Current diabetes reports 2012; 12: 403-413

Tesfaye S, Watt J, Benbow SJ, Pang KA, Miles J, MacFarlane IA. Electrical spinal cord stimulation for painful diabetic peripheral neuropathy. Lancet 1996; 348: 1698-1701

Tefaye S, Vileikyte L, Rayman G, Sindrup SH, Perkins BA, Baconja M, Vinik Al, Boulton AJM. Painful diabetic peripheral neuropathy: consensus recommendations on diagnosis, assessment and management, Diabetes Metab Res Rev 2011; 27: 629-638 
van Buyten JP, Al-Kaisy A, Smet I, Palmisani S, Smith T. High-Frequency Spinal Cord Stimulation for the Treatment of Chronic Back Pain Patients: Results of a Prospective Multicenter European Clinical Study. Neuromodulation 2012. doi: 10.1111/ner.12006

Verkes RJ, Vanderiet K, Vertommen H, van der Kloot WA, van der Meij J. De MPQ-DLV, een standaard nederlandstalige versie van de McGill Pain Questionnaire voor België en Nederland. In: Van der Kloot WA, \& Vertommen $\mathrm{H}$ (Eds.), De MPQ-DLV, een standaard nederlandstalige versie van de McGill Pain Questionnaire: Achtergronden en handleiding (p. 57-73). Lisse: Swets \& Zeitlinger, 1989 


\section{Chapter 5}

\section{Spinal cord stimulation with hybrid lead relieves pain}

\section{in low back and legs}

Cecile C de $\operatorname{Vos}^{1,2,3}$, Cindy Dijkstra ${ }^{1}$, Mathieu WPMLenders ${ }^{1,3}$, Jan Holsheimer ${ }^{2}$

1. Department of Neurosurgery, Medisch Spectrum Twente Hospital, Enschede, The Netherlands;

2. MIRA - Institute for Biomedical Technology and Technical Medicine, University of Twente, Enschede, The Netherlands;

3. Stichting the Neurobionics Foundation, Enschede, The Netherlands

Neuromodulation 2012; 15: 118-123 


\section{ABSTRACT}

Objective: The failed back surgery syndrome (FBSS) is the most common chronic pain syndrome. Whereas it is relatively easy to achieve pain relief in the lower limbs of FBSS patients with spinal cord stimulation (SCS), it is difficult to manage low-back pain with SCS. The performance of an SCS lead that can be inserted surgically as well as percutaneously (a hybrid lead) was evaluated in a prospective study on the relief of low-back pain and leg pain in patients with FBSS.

Methods: Patients with FBSS being eligible for SCS were enrolled in the study and a hybrid lead was placed surgically. Outcome measures included pain scores for low-back and leg pain assessed by VAS, pain medication and patient satisfaction. These scores were assessed before and at regular intervals after implantation.

Results: It was shown that a single hybrid lead, generally positioned over the physiological midline of the spinal cord, is capable of alleviating both low-back and leg pain in patients with FBSS. 45 subjects were eligible for SCS and received trial stimulation. 42 of them had a successful trial period and were converted to a permanent system. Their average VAS score at baseline was 8.0 for lower limb pain and 7.5 for low-back pain. After 6 months of SCS these average VAS scores were reduced to 3.2 and 3.5, respectively, and also pain medication was reduced significantly.

Conclusion: SCS with a hybrid lead in subjects with FBSS is safe and causes significant pain relief in both the low-back and the lower limbs. 


\section{INTRODUCTION}

Failed Back Surgery Syndrome (FBSS) represents a combination of radicular pain in one or two legs and axial lower back pain of nociceptive and neuropathic origin [1]. Spinal cord stimulation (SCS) is often useful to relieve this chronic, otherwise intractable pain $[2,3]$. SCS is applied to the dorsal side of the spinal cord with a lead implanted in the dorsal epidural space close to the dura mater. Two types of implantable lead designs can be distinguished: percutaneous leads and surgical (or paddle) leads $[4,5,6]$.

Percutaneous leads are small and cylindrical (approximately $1 \mathrm{~mm}$ in diameter) and are inserted by a minimally invasive technique in the dorsal epidural space, employing an epidural needle. Yet, control of its positioning and maintenance of the lead at the right position is difficult. These leads generally have 4 or 8 cylindrical electrode contacts with centre distances of 4 to $9 \mathrm{~mm}$, positioned near the tip of the lead.

Surgical leads have a tip that is paddle shaped and several mm wide. Generally 4 to 16 disc electrode contacts are positioned at equal distances on the ventral side of the paddle, sometimes in two or more longitudinal rows. The disc electrodes just on the ventral side of the lead, causes electric current to flow primarily ventrally from the paddle lead to the epidural space, the dura mater and the CSF towards the dorsal spinal cord. This way, waste of energy is minimized. Additionally, due to the larger mass of its paddle, the distance of the electrodes to the spinal cord is generally smaller, thus improving the extent and intensity of the paraesthesia and reducing energy consumption $[4,6,7]$.

The implantation of a paddle lead requires a laminotomy or laminectomy, which are both more invasive than the percutaneous procedure. However, according to several studies, a paddle lead provides greater stability, has less tendency to migrate in the epidural space, and has better long term coverage than a percutaneous lead [5,8,9]. A study by Rosenow et al. [10] showed for both types of lead comparable numbers for migration and coverage problems, but paddle leads were relatively small and used primarily when technical difficulties arose. 
Whereas in FBSS patients lower limb pain can be alleviated easily with SCS, it is more difficult to achieve sufficient pain relief in the low-back region $[11,12,13,14]$. In FBSS patients the optimal position of the stimulating contact (cathode) varies between the rostral end of T7 and the caudal end of T10 [15]. To stimulate the lower limbs, the stimulating contact should be around vertebral level T9-T10. However, to target the low-back, the cathode should be placed more rostrally $[15,16,17]$. To target both regions, several techniques have been proposed, including: stimulation with a $2 \times 4$-electrodes paddle lead [15], with a 16-electrodes paddle lead, or with two 8-electrodes paddle leads [18], stimulation with one percutaneous 8-electrodes lead anchored over the midline [19], and stimulation with two side-by-side placed, independently programmable percutaneous 4-electrodes leads (dualchannel stimulation) [20].

Modelling experiments by Holsheimer et al. [21,22,23] and clinical studies by North et al. $[13,24]$ have shown that a single lead placed over the physiological midline of the spinal cord and programmed with a guarded cathode would generally perform better in targeting the low-back than dual lead configurations.

A hybrid lead combines the advantages of a single, midline, surgical lead (stability, mass) and those of a percutaneous lead (simple implantation). Therefore, the Lamitrode S8 (model 3286, St. Jude Medical, Plano, TX) was used (see Figure 1). This lead is shaped like a small paddle (67mm long, $4 \mathrm{~mm}$ wide and $1.8 \mathrm{~mm}$ thick) and contains 8 electrode contacts with 7 $\mathrm{mm}$ centre-to-centre spacing. In order to evaluate the hybrid lead, its performance in FBSS patients was investigated in a prospective clinical study and the outcome measures were compared with other studies on FBSS patients. The primary outcome measure of this study was pain reduction both in the low back and the lower limbs of FBSS patients after 6 months and 1 year of SCS. 


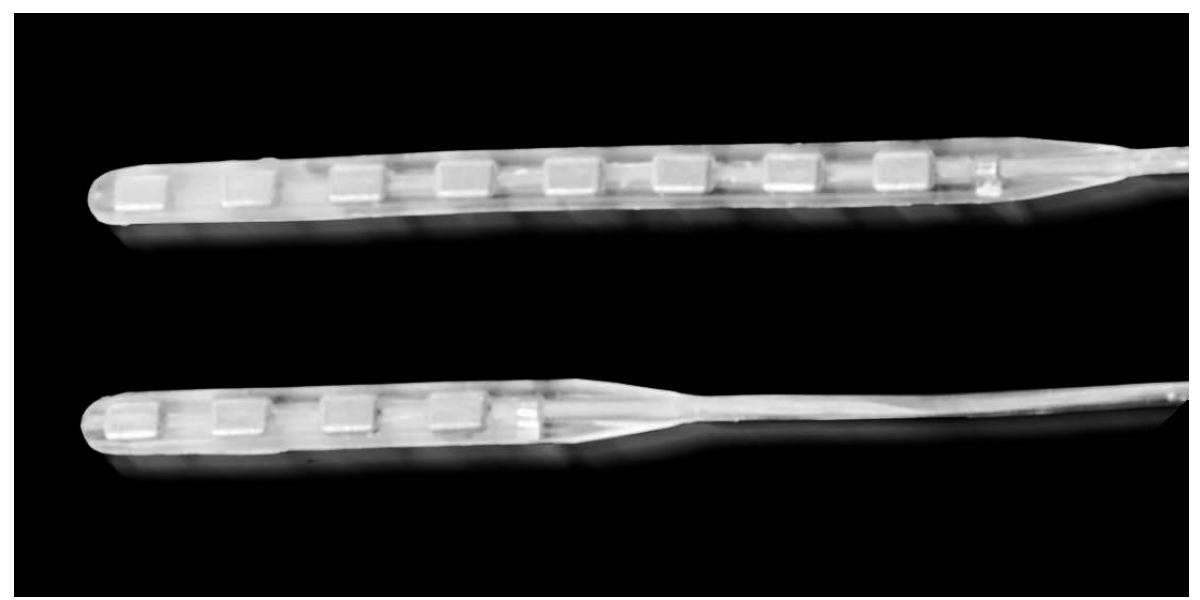

Figure 1. The new hybrid leads: Lamitrode S4 (model 3243) and Lamitrode S8 (model 3286), with 4 and 8 electrode contacts respectively. The individual contacts are $4 \mathrm{~mm}$ long and $2.5 \mathrm{~mm}$ wide, with a centre-to-centre spacing of $7 \mathrm{~mm}$ (St Jude Medical, Plano, TX).

\section{METHODS}

\section{Patients}

Subjects with FBSS visiting our clinic in 2008 and 2009 and being eligible for SCS according to inclusion and exclusion criteria developed by the Dutch Neuromodulation Study Group were enrolled in the study. Subjects with (traumatic) brain injury or psychological or cognitive problems were excluded. All participating subjects gave informed consent prior to enrollment. Subjects were only included in the study when they suffered from both lowback and lower-limb pain. The presumably neuropathic pain had to be present for more than a year and the visual analogue scale (VAS) score for pain should be 5 or more for both low-back and legs. The Institutional Ethics Review Board waived the need for medical ethical assessment.

The majority of the patients has been using medication like paracetamol, NSAIDs, opioids or neurotropic medication such as antidepressants and anticonvulsants, for several years and underwent various other therapies like transcutaneous electrical nerve stimulation (TENS) and lumbar sympathetic ganglion and/or RIS-blocks, in attempts to alleviate their pain. However, these medication and therapies did not provide sufficient pain relief and additionally caused undesirable side effects. Due to serious side effects, some patients were not using pain-medication anymore at the start of our study. 


\section{Lead implantation}

The lead was placed in the low-thoracic epidural space via a 10 Gauge epidural needle. The needle, inserted $1.5 \mathrm{~cm}$ lateral from the midline at level L4-L5, entered the epidural space through a unilateral fenestration at vertebral level L2-L3. Although intralaminar fenestration was not strictly necessary, it was performed to ensure that the lead would be placed below the epidural fat and in direct contact with the dura, without penetrating the dura. The lead was positioned in the epidural space in a way that the paraesthesia corresponded to the patient's painful body area. When the lead was positioned correctly, it was secured at the midline to the fascia to prevent migration. To prevent infections, ciproxin and augmentin were both given prophylactically to all patients, unless a patient was known to be allergic to either medication.

All subjects included in the study had a trial stimulation period prior to the implantation of a permanent system. During trial stimulation the lead was connected via an extension cable to a handheld external pulse generator that allowed the patient to adjust the intensity of the stimulation to the level required. The trial period lasted about one week and was used to determine paraesthesia coverage and pain relief. An SCS trial was considered successful when a patient reported at least $50 \%$ reduction of pain in legs and low-back.

After a successful trial, the lead used for the trial was connected to a implantable, constant current, non-rechargeable pulse generator (EonC ${ }^{\mathrm{TM}}$ or Genesis XP ${ }^{\mathrm{TM}}$, St Jude Medical, Plano, TX). The stimulation configurations were tested according to a structured protocol to ensure that no possibly relevant configurations would be missed. The configurations were programmed and tested according to the patient's description of the pain and paraesthesia. We used body maps for the patients to indicate their pain areas and paraesthesia areas and we aimed for an optimal overlap of the paraesthesia areas with the painful dermatomes. This could require alternating stimulation with 2 different electrode contact configurations.

\section{Study design}

Outcome measures included VAS scores for low-back and leg pain separately, pain medication, preferred contact configurations and patient satisfaction. The VAS and medication scores were assessed immediately before and after implantation, and after 3, 6 and 12 months of SCS. The VAS scores were analysed in such a way that we could compare our results with other studies on SCS in FBSS. Our primary outcome measures were pain relief after 6 months 
and 1 year of SCS. These study endpoints were obtained in each patient at a different time, since all patients were included during a period of two years. To check whether changes in pain perception might occur after 6 months of stimulation, the patients are still observed after the study endpoint.

The VAS score for pain depends on the overlap of the painful body area and the paraesthesia area and on the effectiveness of the paraesthesia to suppress the pain. The perceived paraesthesia relies first and foremost on the electrode position and contact configuration, but also on the stimulation amplitude, stimulation frequency and pulse width. All these stimulation parameters were adjusted and optimized for the individual patient during their follow up visits, and their VAS scores for pain and other outcome measures were noted.

Output data were reported as group mean values and paired t-tests were used to analyse the results. A two-tailed $p$-value of $<0.05$ were considered statistically significant. Only those patients with successful trial stimulation were included in the analysis.

\section{RESULTS}

\section{General}

Forty-five patients, with at least one failed surgical back procedure, were eligible for SCS and included in the study. They all received trial stimulation and 42 patients (93\%) had a successful trial period. In 3 patients the trial period was not successful. They felt the paraesthesia in the right area, but they still felt the pain as well as the paresthesia, resulting in a pain reduction of less than $50 \%$. One other patient, who experienced more than $80 \%$ pain reduction during the trial period, got an infection shortly after implantation which resulted in explantation of the SCS system. The remaining 41 patients (20 men, 21 women, 33-76 years old, mean age at time of enrolment 56 years) completed their trial period successfully and continued with the permanent SCS system. All these patients were followed for at least 12 months.

Lead migration occurred in one case during follow up. To retrieve effective paraesthesia in this patient, a percutaneous lead (octrode, St Jude Medical, Plano, TX) was added, connected to the yet implanted pulse generator and used for stimulation as well. Two other patients already received an additional percutaneous lead during trial stimulation, since only unilateral paraesthesia coverage was obtained with one S8 lead in these patients. 
Besides this one infection shortly after implantation and one lead migration two months after implantation, there were no other adverse events.

\section{VAS scores}

Pain reduction for low-back and legs, respectively, was obtained after implantation of the system and during all follow up visits. As shown in Figures $2 a$ and $2 b$, an average reduction of more than $50 \%$ for both low-back and lower limbs was obtained at all follow up visits up to and including 6 months. The average VAS score at baseline was 8.0 for leg pain and 7.5 for low-back pain $(n=41)$.

A

VAS legs

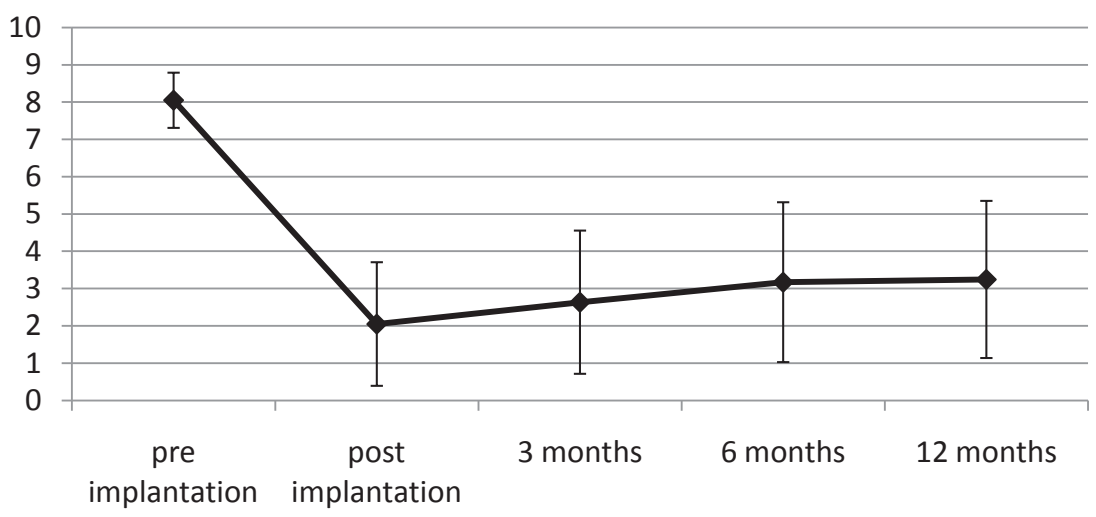

B

VAS back

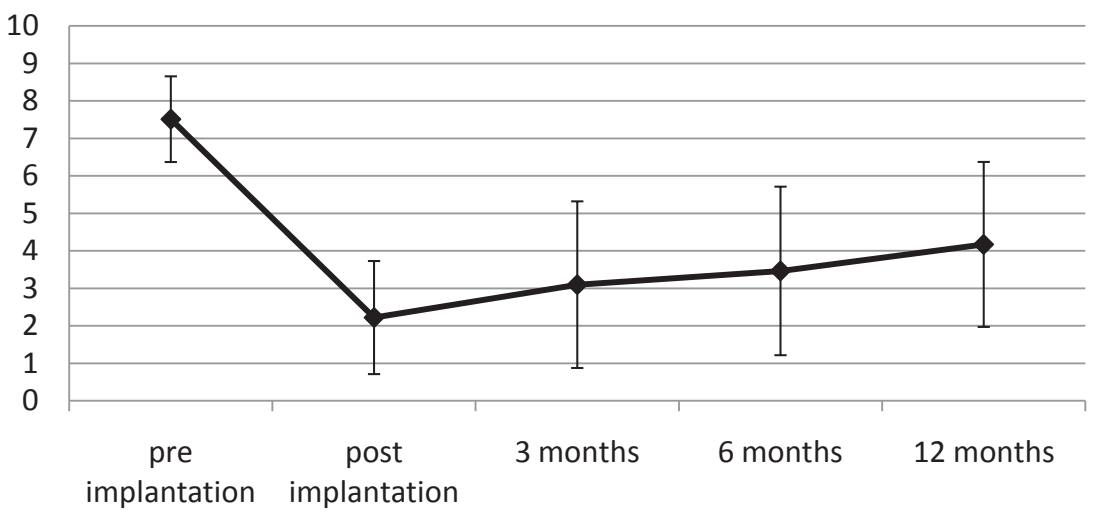

Figure 2. Average VAS scores for pain in (a) legs and (b) low-back of 41 FBSS patients stimulated with a hybrid lead (lamitrode S8); pain scores were assessed before and after permanent implantation and after 3, 6 and 12 months of stimulation. Error bars represent the standard deviation. 
After 6 months of SCS these average scores were reduced to 3.2 and 3.5, respectively, and both pain reductions are statistically highly significant $(p<0.001)$. After 6 months, 4 patients $(10 \%)$ were completely pain free. A pain reduction of more than $50 \%$ in legs and low back was obtained in $73 \%$ and $56 \%$ of the patients, respectively (Figure $3 \mathrm{a}$ and $3 \mathrm{~b}$ ). Two patients indicated to experience no pain reduction in their legs and 4 patients experienced no effects in their low back.

A

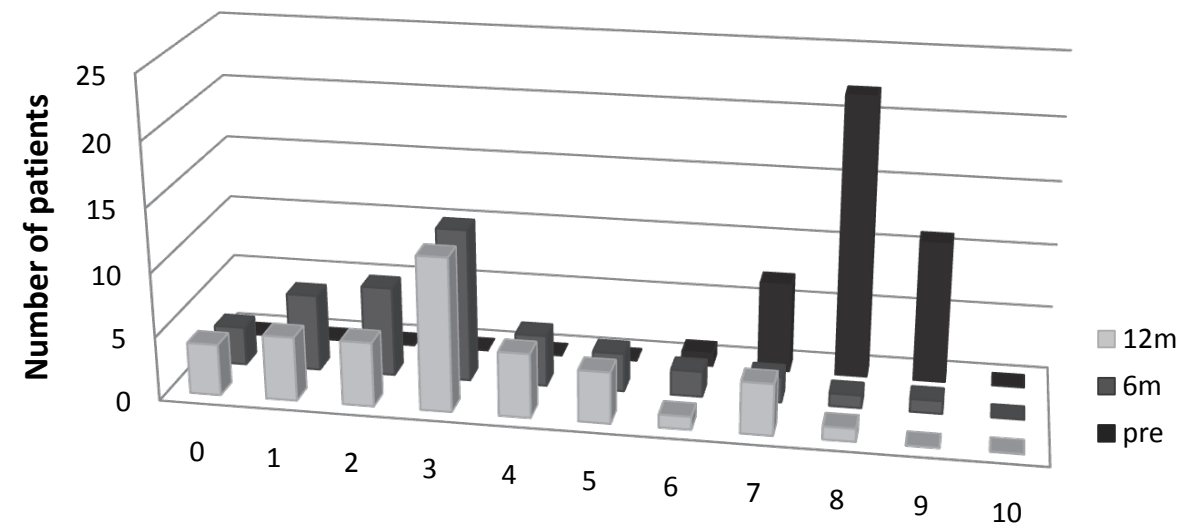

VAS scores legs

B

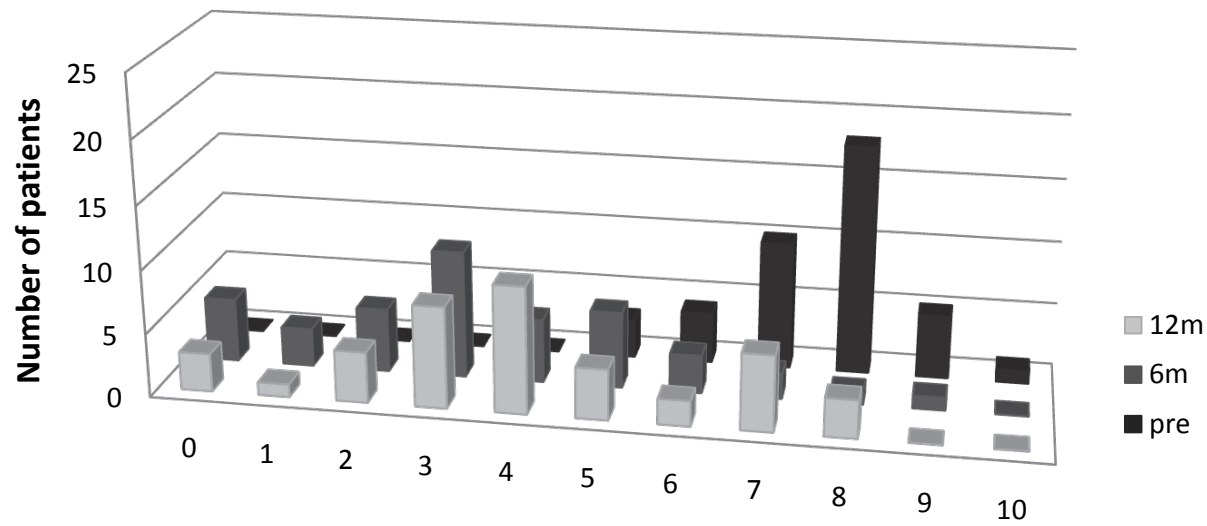

VAS scores back

Figure 3. The number of patients per VAS scores for pain in (a) legs and (b) low-back; the depicted pain scores were assessed before implantation (black, back row), after 6 months of SCS (gray, middle row) and after 12 months of SCS (light gray, front row). 
After 12 months the average score for leg pain was still 3.2 while the average score for low-back pain was increased to 4.2. At that time, $71 \%$ of the patients experienced a pain reduction of more than $50 \%$ in their legs and $51 \%$ of the patients had over $50 \%$ reduction of their low-back pain. Three patients still indicated to be completely pain free, while 2 others had no pain reduction in their legs and 4 patients experienced no effects in their low-back.

\section{Pain Medication}

Due to serious side effects, 7 patients did not use any pain medication at the start of our study. On the other hand, prior to SCS treatment oral opioids were used by 24 patients, neurotropic medication by 18 patients, NSAIDs by 14 patients and paracetamol by 12 patients. So, 21 patients used a combination of two or more different drugs and yet none of the included patients experienced sufficient pain relief from the medication they used.

As a result of 6 months of SCS, 23 patients were able to reduce their pain medication. They either reduced the dose or stopped with one type or with all medication they were using, see Figure 4. After 6 months, SCS had become the sole pain treatment for 17 patients. After 12 months of SCS, 25 patients had reduced their pain medication compared to the situation before implantation and 18 patients did not use any pain medication anymore. The alleviating effects of SCS on the neuropathic pain and the intake reduction of pain medication made that other causes of pain, like acquired hip dysplasia and osteoarthritis, became manifest in 5 patients. 


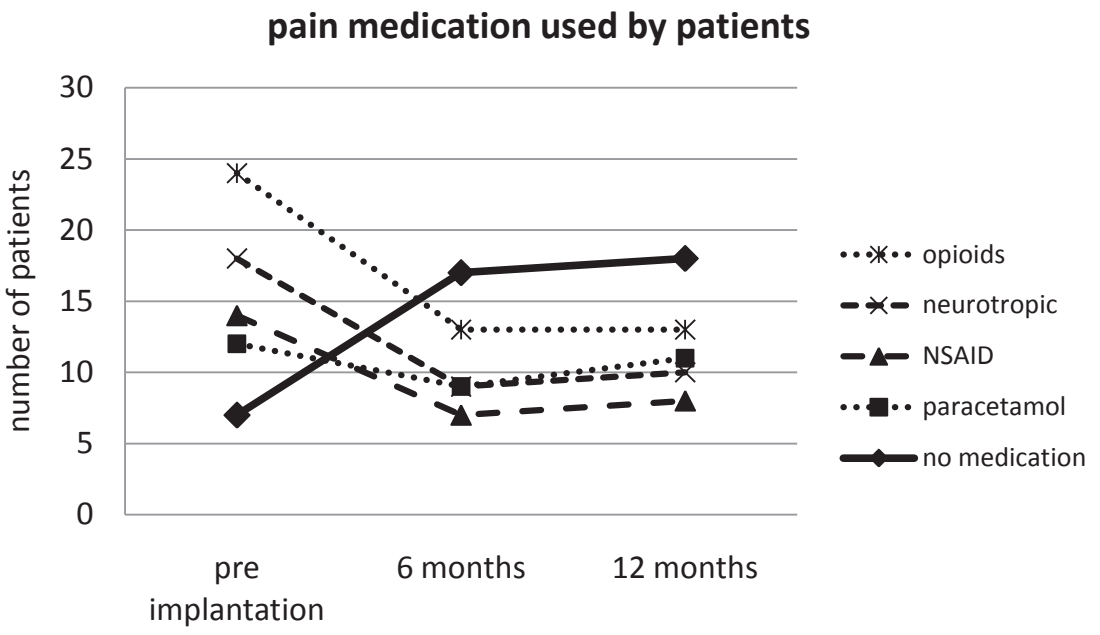

Figure 4. The number of patients using different types of pain medication prior to SCS treatment and after 6 and 12 months $(n=41)$ of SCS treatment.The solid line indicates the number of patients not using any pain medication. Change in intake dose is not visible in this figure.

\section{Contact configurations, paraesthesia and patient satisfaction}

Of the 41 subjects, 38 subjects (93\%) had just one hybrid lead (lamitrode S8) implanted. All 38 obtained good paraesthesia coverage with this lead. The tip of the lead was generally placed between T7 and T9; most patients preferred the tip of the lead positioned at T8. Obtaining adequate paraesthesia and pain relief in both low-back and legs required a double stimulation set in 12 patients. A double stimulation set is stimulation with two alternating electrode configurations. Usually, one configuration covered the back and one configuration covered the legs or each configuration covered one side of the body.

Guarded cathode configurations (+ - + or + - + + ) and narrow bipoles (+ -) were selected most frequently for chronic stimulation. 23 Patients preferred guarded cathodes, 18 patients a narrow bipole and 7 patients had another type of electrode contact configuration. Seven patients could select more than one contact configuration as they preferred different configurations during different physical activities.

All subjects coloured their pain areas and paraesthesia areas on body maps. On average, the paraesthesia-area covered more than $80 \%$ of the painful body areas of the patients, of which the legs were $100 \%$ covered while the low-back had coverage of $70 \%$. 
Patients were asked to rate the quality of their life before implantation and after one year of SCS therapy; 0 would be the most miserable and 100 the best possible life. Before implantation the patients on average rated their life 34. After one year of stimulation the average score was 70 . In addition, 36 of the 41 patients would recommend this therapy to other patients and would choose again for an SCS system in case they had to make the decision once more.

\section{DISCUSSION}

\section{Pain and Pain Medication}

Spinal cord stimulation with a hybrid lead (lamitrode S8) gave a significant reduction of pain in both the low back and legs of patients with FBSS. 41 FBSS patients received an SCS system and all patients obtained on average good results at all follow up visits. However, 6 patients experienced only minimal or no pain relief after 12 months of SCS. This group includes the 5 patients with other aetiologies of pain.

Prior to SCS treatment, the pain caused by FBSS was dominating other affections, like acquired hip dysplasia or osteoarthritis, or the pain medication used, though insufficient to relieve the neuropathic pain, did suppress the pain caused by the other affections. These 5 patients are using analgesic drugs again and they are treated for their other affections.

After 6 months of SCS, pain medication intake was reduced in 23 patients of whom 17 stopped with all pain medication. After 12 months, the change in medication intake seems to be stabilized. Particularly the use of opioids and neurotropic medication was reduced considerably during the first 6 months of SCS treatment, resulting in less medication induced side effects and an improved quality of life. However, in some subjects there was an increase of pain indicated during the second 6 months of SCS treatment, especially in the low back. Some of these patients have chosen to perceive somewhat more pain but being released from the disabling side effects of the medication, over feeling less pain but experiencing the side effects.

\section{VAS scores}

Other observational studies have also examined new SCS techniques for the treatment of FBSS [3]. However these studies are rather heterogeneous and so are the outcome measures these studies specified. 
Van Buyten et al. [20] implanted 2 percutaneous leads in 17 patients with FBSS and found an average VAS decrease of 5.1 points for lower limb pain and of 3.7 points for back pain. In our study the average VAS score for leg pain decreased by 4.8 VAS points (from 8.0 to 3.2 ) and the average score for low back pain decreased by 4.0 VAS points (from 7.5 to 3.5).

In a study by North et al. [13] 21 patients were implanted with two percutaneous leads. They defined pain relief as more than $50 \%$ pain reduction. After 6 months, 16 patients were still using SCS, 8 of them reported relief of low back pain and 14 reported relief of leg pain. After 2 years of follow up, still 13 patients continued stimulation, of whom 7 patients reported relief of low back pain and 12 patients reported relief of leg pain. So, a significant number of patients was lost during follow up and 6 had stopped using the stimulation. After 1 year of follow up, we have not lost any patient yet and still all 41 patients use the stimulation with $51 \%$ of the patients reporting pain relief for their low back and $71 \%$ of the patients reporting relief of their leg pain.

Barolat et al. [18] used surgical paddle leads in patients with low back pain and they showed a reduction of the VAS score for pain in $83 \%$ of the patients after 6 months and in $69 \%$ of the patients after 12 months. This included any VAS reduction for low back pain. Applying this criterion to the patients in our study reveals that $90 \%$ of the patients experience pain reduction in the low back both after 6 and 12 months of SCS. Our results from stimulation with just one hybrid lead are comparable or compare favourably to earlier studies of FBSS patients treated with SCS [3].

\section{Contact configurations, paraesthesia and patient satisfaction}

Modelling experiments by Holsheimer et al. [21,22,23] have shown that guarded cathode configurations recruit the largest number of dorsal column fibres and therefore have better paraesthesia coverage and potentially the strongest analgesic effects. Though there was variety in the preferred configurations, the guarded cathode was indeed the most common one.

Scoring pain always requires the participation, insight and accurateness of a patient. In this study patients also needed to differentiate between back pain and leg pain, which could have been difficult for some of them. To avoid debate about boundaries between both body areas, we have asked the patients to indicate their pain-area and paraesthesia-area on a body map at each visit. Although colouring of the body maps by patients is not always very precise, the patients were able to indicate that there was limited change over time in 
paraesthesia-area. The legs were covered completely in all patients, while the low-back had an average paraesthesia coverage of $70 \%$.

Mironer et al. [19] used a single percutaneous lead with 8 electrode contacts and positioned and anchored it across the midline of the spinal cord. With this method they obtained an average coverage of the legs of $89 \%$ and an average coverage of the back of $58 \%$ after a mean follow up period of 9 months. In general the patients in this study were satisfied with their SCS system and 78\% of them rated the quality of their life improved of greatly improved.

Duyvendak [15] performed laminectomy and used a dual quadripolar paddle lead in patients with FBSS. He obtained good back coverage after an average follow up of 19 months in 9 (35\%) of the 26 patients with an implanted SCS system. Additionally Duyvendak [15] asked his patients whether they would repeat the procedure they have had and $85 \%$ answered positively. Also $88 \%$ of our patients answered this question positively. Furthermore, 37 of our patients (90\%) rated the quality of their life as improved or greatly improved. Again the lamitrode $\mathrm{S} 8$ is comparable or compares favourably to previous studies.

\section{Lead implantation and adverse events}

Although the lamitrode S8 lead is suitable for implantation via a 10 Gauge epidural needle, for safety reasons, we have chosen to use assistance of a neurosurgical procedure for all implantations during this study. When we started this study there was no literature available about the performance of hybrid leads. In order to safely place the lead at an optimal position, that is, as close to the dura as possible, we used unilateral interlaminar fenestration in all our patients. During this study we have not changed our implantation procedure.

In the mean time, the Epiducer (St Jude Medical, Plano, TX) has been introduced; a device to insert a lamitrode S8 lead into the epidural space via a percutaneous procedures. This new method of placing the lead does not require a neurosurgical procedure and is therefore hoped to cause less discomfort for the patients and make a faster recovery possible. However, a less invasive method of placing the lead provides no direct vision and may hinder placement of the lead right onto the dura. 
In our study, 42 (93\%) of the 45 eligible patients had a successful trial period of SCS and were converted to a permanent system. SCS is not yet risk free. Taylor et al. [25] reported that $43 \%$ of patients with chronic back and leg pain experienced one or more complications with SCS. The majority of these complications were due to electrode or lead problems (27\%), infections (6\%), IPG problems (6\%), extension cable problems (10\%) and CSF-leaks (7\%). Kumar et al. [26] reported that of 42 patients in their study 19 patients (45\%) experienced a total of 34 SCS-related complications, including everything from lead migration (14\%) to loss of paraesthesia (12\%) and infection or wound break-down (10\%).

In our series only 1 patient (2\%), who experienced more than $80 \%$ pain reduction in both back and legs, got an infection shortly after the trial stimulation period. This resulted in explantation of the SCS system and immediate treatment of the infection. Recently this patient has received a new SCS system and again reaches $80 \%$ pain reduction.

Surgical leads are generally less sensitive to migration than percutaneous leads. Migration of the hybrid lamitrode S8 lead occurred just in 1 case (2\%) two months after implantation. To retrieve effective paraesthesia in this patient, a percutaneous lead (octrode, St Jude Medical, Plano, TX) was added and used for stimulation as well. In 2 other patients only unilateral paraesthesia coverage could be obtained with one S8 lead. Therefore these patients received an additional percutaneous lead directly during trial stimulation. So in 3 patients (7\%) both a hybrid lamitrode S8 and a percutaneous lead had to be placed. Loss of effective paraesthesia without (noticeable) migration of the lead occurred in 4 patients (10\%). There were no other adverse events.

SCS with a hybrid lead in patients with FBSS is safe and causes significant pain reduction in both low back and legs. 


\section{REFERENCES}

1. Onesti ST. Failed Back Syndrome. The Neurologist. 2004;10:259-264.

2. Mailis-Gagnon A, Furlan AD, Sandoval JA, Taylor RS. Spinal cord stimulation for chronic pain. Cochrane Database of Systematic Reviews. 2004;3:Art. No.: CD003783.

3. Frey ME, Manchikanti L, Benyamin RM, Schultz DM, Smith HS, Cohen SP. Spinal cord stimulation for patients with failed back surgery syndrome; a systematic review. Pain Physician. 2009; 12: 379-397.

4. North RB, Lanning A, Hessels R, Cutchis PN. Spinal cord stimulation with percutaneous and plate electrodes: side effects and quantitative comparisons. Neurosurgical Focus. 1997; 2: 1-5.

5. Villavicencio AT, Leveque JC, Rubin L, Bulsara K, Gorecki JP. Laminectomy versus percutaneous electrode placement for spinal cord stimulation. Neurosurgery. 2000; 46: 399-405.

6. Manola L, Holsheimer J. Technical performance of percutaneous and laminectomy leads analyzed by modeling, Neuromodulation. 2004; 7: 231-241.

7. Holsheimer J, Khan YN, Raza SS, Khan EA. Effects of electrode positioning on perception threshold and paresthesia coverage in spinal cord stimulation. Neuromodulation. 2007; 10: 34-41.

8. North RB, Kidd DH, Olin J, Sieracki JM. Spinal cord stimulation electrode design: prospective, randomized, controlled trial comparing percutaneous and laminectomy electrodes - part I: technical outcomes. Neurosurgery. 2002; 51: 381-389.

9. North RB, Kidd DH, Petrucci L, Dorsi MJ. Spinal cord stimulation electrode design: prospective, randomized, controlled trial comparing percutaneous and laminectomy electrodes - part II: clinical outcomes. Neurosurgery. 2005; 57: 990-996.

10. Rosenow JM, Stanton-Hicks M, Rezai AR, Henderson JM. Failure modes of spinal cord stimulation hardware. J Neurosurg Spine. 2006; 5: 183-190.

11. North RB, Ewend MG, Lawton MT, Kidd DH, Piantadosi S. Failed back surgery syndrome: 5-year follow-up after spinal cord stimulator implantation. Neurosurgery. 1991; 28: 692-699.

12. Dario A, Fortini G, Bertollo D, Bacuzzi A, Grizzetti C, Cuffari S. Treatment of failed back surgery syndrome. Neuromodulation. 2001; 4: 105-110

13. North RB, Kidd DH, Olin J, Sieracki JM, Farrohki F, Petrucci L, Cutchis PN, Spinal cord stimulation for axial low back pain, a prospective, controlled trial comparing dual with single percutaneous electrodes. Spine. 2005; 30: 1412-1418.

14. Oakley JC. Spinal Cord Stimulation in axial low back pain: solving the dilemma, Pain Medicine. 2006; 7 : S58-S63.

15. Duyvendak W. Spinal cord stimulation with a dual quadripolar surgical lead placed in general anesthesia is effective in treating intractable low back and leg pain. Neuromodulation. 2007; 10: 113-119.

16. Barolat G, Massaro E, He J, Zeme S, Ketcik B. Mapping of sensory responses to epidural stimulation of the intraspinal neural structures in man. Journal of Neurosurgery. 1993;78:233-239.

17. Holsheimer J, Barolat G. Spinal geometry and paresthesia coverage in spinal cord stimulation, Neuromodulation. 1998; 1: 129-136.

18. Barolat G, Oakley JC, Law JD, North RB, Ketcik B, Sharan A. Epidural spinal cord stimulation with a multiple electrode paddle lead is effective in treating intractable low back pain. Neuromodulation. 2001; 4: 59-66.

19. Mironer YE, Satterhwaite JR, Lewis EM, Haasis JC, LaTourette PC, Skoloff EM, Diaz RL. Efficacy of a single, percutaneous, across midline, octrode ${ }^{\circledR}$ lead using a "midline anchoring" technique in the treatment of chronic low back and/or lower extremity pain: a retrospective study, Neuromodulation. 2008; 11: 286-295

20. van Buyten JP, van Zundert J, Milbouw G. Treatment of failed back surgery syndrome patients with low back and leg pain: a pilot study of a new dual lead spinal cord stimulation system, Neuromodulation. 1999; 2: 258-265.

21. Holsheimer J, Wesselink WA. Effect of anode-cathode configuration on paresthesia coverage in spinal cord stimulation. Neurosurgery. 1997; 41: 654-660.

22. Holsheimer J. Effectiveness of spinal cord stimulation in the management of chronic pain: analysis of technical drawbacks and solutions. Neurosurgery. 1997;40:990-996.

23. Holsheimer J. Does dual lead stimulation favor stimulation of the axial lower back?, Neuromodulation. 2000; 3: 55-57. 
24. North RB, Kidd DH, Olin J, Sieracki JN, Petrucci L. Spinal Cord stimulation for axial low back pain: A prospective, controlled trial comparing 16-contact insulated electrodes with 4-contact percutaneous electrodes. Neuromodulation. 2006; 9: 56-67.

25. Taylor RS, van Buyten JP, Buchser E. Spinal cord stimulation for chronic back and leg pain and failed back surgery syndrome: a systematic review and analysis of prognostic factors. Spine. 2005; 30: 152-160.

26. Kumar K, Taylor RS, Jacques L, Eldabe S, Meglio M, Molet J, Thomson S, O'Callaghan J, Eisenberg E, Milbouw G, Buchser E, Fortini G, Richardson J, North RB. The effects of spinal cord stimulation in neuropathic pain are sustained: a 24-month follow-up of the prospective randomized controlled multicenter trial of the effectiveness of spinal cord stimulation. Neurosurgery. 2008; 63: 762-770. 



\section{Chapter 6}

\section{Burst spinal cord stimulation evaluated in patients}

\section{with failed back surgery syndrome and painful diabetic}

\section{neuropathy}

Cecile C de Vos ${ }^{1,2,3}$, Marjanne J Bom², Sven Vanneste ${ }^{4,5}$, Mathieu WPM Lenders ${ }^{1,3}$, Dirk de Ridder ${ }^{4,5,6}$

1. Department of Neurosurgery, Medisch Spectrum Twente, Enschede, the Netherlands

2. Department of Clinical Neurophysiology, University of Twente, Enschede, the Netherlands

3. the Neurobionics Foundation, Enschede, the Netherlands

4. Brai²n \& TRI, University Hospital Antwerp, Belgium

5. Department of Translational Neuroscience, Faculty of Medicine, University of Antwerp, Belgium

6. Department of Surgical Sciences, Dunedin School of Medicine, University of Otago, New Zealand.

Submitted to Neuromodulation 


\section{ABSTRACT}

Objective: Spinal cord stimulation (SCS) is used for treating intractable neuropathic pain. Generally, it induces paraesthesia in the area covered by SCS. Burst spinal cord stimulation was introduced as a new stimulation paradigm with good pain relief without causing paraesthesia. Good results have been obtained in patients who were naive to SCS.

In this study we assess the effectiveness of burst stimulation in three groups of chronic pain patients who are already familiar with SCS and the accompanying paraesthesia.

Methods: Forty-eight patients with at least six months of conventional, tonic stimulation tested burst stimulation for a period of two weeks. They were classified in three different groups: a cross-section of our population with painful diabetic neuropathy (PDN), a crosssection of our population with failed back surgery syndrome (FBSS), and FBSS patients who over time had become poor responders (PR) to SCS. Visual analogue scale scores for pain were assessed prior to implantation, with tonic stimulation and after two weeks of burst stimulation.

Results: Burst stimulation reduced pain significantly for almost all patients. When compared to tonic stimulation, burst stimulation led to a significant additional pain reduction of on average $44 \%$ in patients with PDN $(p<.001)$ and $28 \%$ in patients with FBSS $(p<.01)$. Patients from the PR group benefitted less from burst stimulation on average. In addition, burst stimulation caused little or no paresthesia whereas tonic stimulation did induce paraesthesia. Most patients preferred burst stimulation, but several preferred tonic stimulation because the paraesthesia assured them that the SCS was working.

Conclusion: About $60 \%$ of the patients with tonic spinal cord stimulation experienced further pain reduction upon application of burst stimulation. 


\section{INTRODUCTION}

Spinal cord stimulation (SCS) is an invasive technique that administers electrical stimulation to the dorsal columns of the spinal cord to reduce pain perception. SCS has shown to be an effective treatment for various neuropathic pain conditions [1-7].

To achieve the most beneficial pain relief for an individual patient, spinal cord stimulation parameters like the configuration of active electrodes, the stimulation frequency, pulse width and pulse amplitude can be adjusted to the patient's needs. The electrical stimulation of the large-diameter fibres in the dorsal column elicits tingling sensations (paraesthesia) in most patients $[8,9]$. The perception and appreciation of this paraesthesia varies to a great extent among patients and is heavily influenced by the stimulation parameters used.

The frequencies of SCS that are most often used in the clinic are around $50 \mathrm{~Hz}$ and generally vary between 30 and $120 \mathrm{~Hz}$. New types of stimulation paradigms for SCS using high frequency stimulation up to $10 \mathrm{kHz}$ have been introduced $[10,11]$. Recently burst stimulation was introduced as a new stimulation paradigm $[12,13]$ combining features of high frequency stimulation with conventional, tonic stimulation. The burst stimulation used provided pulse trains of 5 high frequency pulses at $500 \mathrm{~Hz}$ ( $=500 \mathrm{~Hz}$ spike frequency) occurring 40 times a second ( $=40 \mathrm{~Hz}$ burst frequency). The pulse width was fixed at $1 \mathrm{~ms}$ and the amplitude was optimized for each individual patient. Burst stimulation could be programmed in standard Eon Implantable Pulse Generators (IPG) (St. Jude Medical, Plano, TX). Burst stimulation was tested during a one month trial stimulation period in twelve patients who were naive to SCS. The pain reduction obtained with burst stimulation was comparable or compared favourably to tonic stimulation and paraesthesias were, in contrast to tonic stimulation, barely present [12]. In a subsequent randomized placebo controlled trial in fifteen patients burst stimulation demonstrated to be significantly better than placebo stimulation and better for global pain than tonic stimulation [13].

The goal of this study is to assess the effectiveness of burst stimulation in chronic pain patients who are already receiving SCS treatment for at least six months. This is a challenging group, because these patients are used to feeling paraesthesias when the spinal cord stimulation is active. It can be expected that these patients are conditioned to associate pain reduction with paraesthesia, and will report lesser gains in pain reduction upon the introduction of paraesthesia-free stimulation when compared to patients who never experienced SCS. 


\section{METHODS}

Patients with an Eon IPG (St. Jude Medical, Plano, TX) and using tonic spinal cord stimulation for at least six months tested burst stimulation for 2 weeks. Three groups of patients were included; two groups of patients who participated in previous studies, that is patients with painful diabetic neuropathy (PDN) and patients with low back and leg pain (failed back surgery syndrome, FBSS [14]), and one group of patients with low back and leg pain (failed back surgery syndrome) who over time had started to experience insufficient effect of tonic stimulation (poor responders: PR). The study conformed to the Declaration of Helsinki and was approved by the Institutional Review Board Twente. All patients gave written informed consent.

Prior to implantation of the SCS system all patients underwent a psychological screening and filled out several questionnaires about their pain and quality of life, like Symptom Checklist 90, McGill pain questionnaire, EQ5D and Rand SF 36. These data were used to define the baseline situation of the patients.

To acquire the data on tonic stimulation, patients visited the hospital and filled out questionnaires about their pain and experiences with tonic stimulation. Burst stimulation was programmed with settings similar to those used before ( 5 spikes at $500 \mathrm{~Hz}$ spike mode, $40 \mathrm{~Hz}$ burst mode, $1 \mathrm{~ms}$ pulse width) [12] and amplitude was set at $90 \%$ of the paraesthesia threshold. The patients evaluated the burst stimulation for two weeks, which was double the evaluation time used in previous studies in patients naive to SCS $[12,13]$. During the evaluation period patients were at home and kept a diary about their pain and its impact on daily life. After two weeks patients visited the hospital again, filled out questionnaires about their pain and experiences with burst stimulation and were asked whether they preferred to either return to tonic stimulation or keep burst stimulation.

Pain scores were acquired for feet, legs and back separately for both tonic and burst stimulation conditions. Patients from the three groups had pain in various body parts. To be able to compare the different groups to each other, the highest pain score for any of their body parts was used for analyses. Within a group we assessed the effect of burst stimulation on feet, legs and back separately. 
To relate the electrical current output of tonic and burst stimulation in our patients to the electrical current in previous studies and modelling experiments $[12,13,15]$ two quantities were determined by: the charge per stimulation pulse, calculated as the product of the current amplitude [Ampere] and pulse duration [seconds], and the stimulation current, calculated as the product of the charge per pulse [Coulomb] and the number of pulses per second. The data was normally distributed and paired samples t-tests were performed within each group of patients to detect statistically significant changes in current delivery.

A repeated measures ANOVA was conducted with stimulation (baseline, tonic and burst stimulation) as within-subjects variable and three different groups of patients (painful diabetic neuropathy (PDN), failed back surgery syndrome (FBSS) and poor responders to conventional tonic stimulation (PR)) as a between-subjects variable. Within each group of patients paired samples t-tests were performed to test whether there were statistically significant changes in pain perception due to burst stimulation as compared to tonic stimulation. In addition, paired samples t-tests were performed to compare the VAS scores between tonic and burst stimulation on respectively the pain patients perceived in their feet, legs and back for three different groups of patients (PDN, FBSS and PR) separately.

\section{RESULTS}

\section{Study population}

Forty-eight patients from Medisch Spectrum Twente Hospital with a SCS system and tonic stimulation participated in the study. Their average age was 56 years ( $S D=9$; range: 29-80 years) and they had on average been experiencing pain for 10 years ( $S D=6$; range: 2-30 years). All patients had been using tonic stimulation for at least 6 months ( $m e a n=2.5$ years, $S D=2.6$, range: $0.5-18$ years) and evaluated burst stimulation for a two week period.

Three different groups of patients were included, 12 patients with PDN, 24 patients with FBSS and 12 patients belonged to the PR group (Table 1). Both the PDN group and the FBSS group represented a cross section of the patient population who had an IPG implanted in Medisch Spectrum Twente Hospital. The poor responders group is a group of patients with low back and leg pain (FBSS) who had a successful trial stimulation period, but had reversion of their pain over time. This pain could not be ameliorated anymore by adjusting tonic stimulation. For this group, burst stimulation was the last option that could be offered. 
Table 1. Overview of the patients of the three groups, including the average age, average duration of pain and spinal cord stimulation and average pain scores.

\begin{tabular}{llllllll}
\hline & Gender & Age & Duration Pain & Duration tonic SCS & \multicolumn{3}{c}{ General Pain scores VAS } \\
\hline & M / F & years & years & years & Baseline & Tonic & Burst \\
PDN & $6 / 6$ & 57 & 9 & 1.8 & 70 & 28 & 16 \\
FBSS & $12 / 12$ & 59 & 11 & 2.9 & 82 & 49 & 35 \\
PR & $4 / 8$ & 50 & 9 & 2.4 & 82 & 74 & 64 \\
\hline
\end{tabular}

SCS (spinal cord stimulation), PDN (painful diabetic neuropathy), FBSS (failed back surgery syndrome), PR (poor responders)

\section{Pain reduction}

Both tonic stimulation and burst stimulation led to pain reduction in almost all patients. Statistical analysis revealed that overall a significant effect was obtained for stimulation $(F=66.3 ; p<.001)$. Tonic stimulation caused an average reduction of $37 \%$ in VAS score in comparison to the baseline situation. Burst stimulation caused a $25 \%$ further pain reduction compared to tonic stimulation which resulted in an average reduction of $52 \%$ in VAS score in comparison to the baseline situation. Figure 1 shows the average pain patients perceived prior to stimulation, with tonic and with burst stimulation.

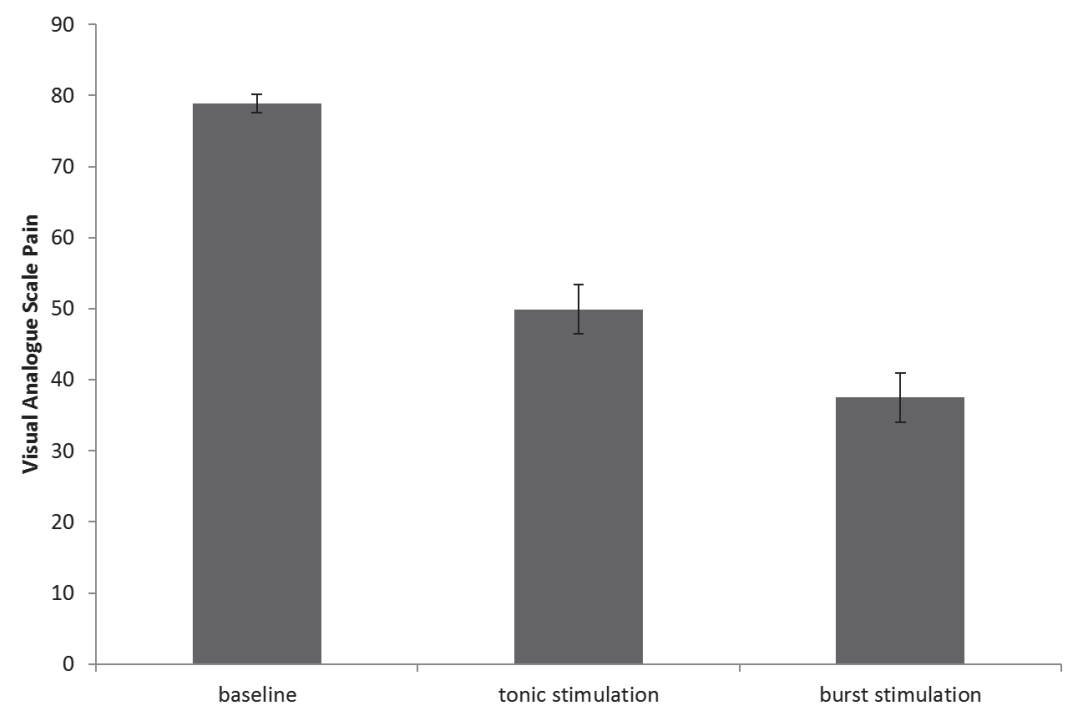

Figure 1. The average visual analogue scale (VAS) scores for pain for all 48 patients assessed preoperative, with tonic stimulation and with burst stimulation respectively. Error bars represent standard errors. 
Pain reduction however was strongly dependent on aetiology (i.e. PDN, FBSS and PR). The effect for burst stimulation was strongest for PDN (decrease of 77\%), followed by FBSS (decrease of $57 \%$ ) and PR (decrease of $23 \%$ ) in comparison to the baseline. A comparison between tonic stimulation and the baseline also revealed the strongest pain reduction for PDN (decrease of 58\%), followed by FBSS (decrease of $41 \%$ ) and PR (decrease of $10 \%$ ). Figure 2 shows the average VAS scores for pain of the three groups of patients for the baseline, tonic and burst stimulation respectively.

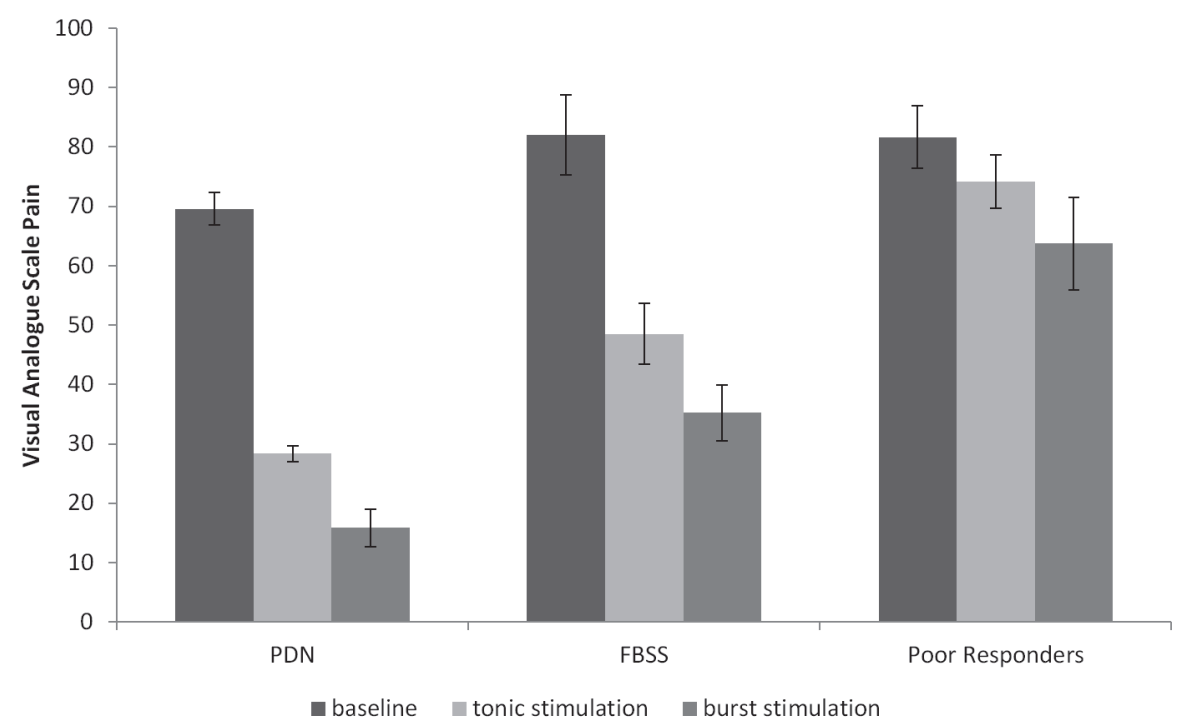

Figure 2. VAS scores for pain of the three groups of patients (painful diabetic neuropathy (PDN), failed back surgery syndrome (FBSS) and poor responders (PR)). Scores are assessed pre-operative, with tonic stimulation and with burst stimulation. Bars represent the average pain score for all patients in a group, error bars represent standard errors.

\section{Painful diabetic neuropathy}

The twelve patients with PDN had an average pain score of 70 prior to implantation. Tonic stimulation reduced their average pain score to 28 which was a significant reduction compared to baseline (see Table 2). After two weeks of burst stimulation the average pain score was significantly further reduced to 16 . Eight out of twelve patients $(67 \%)$ had extra pain reduction with burst stimulation as compared to tonic stimulation. One patient however experienced some pain increase comparing burst to tonic stimulation. Patients with PDN primarily had pain in their feet (see Figure 3). Pain relief by burst stimulation is 
also predominantly in the feet, on average a further $50 \%(p<.05)$. No statistically significant effects were obtained for the legs or the back.
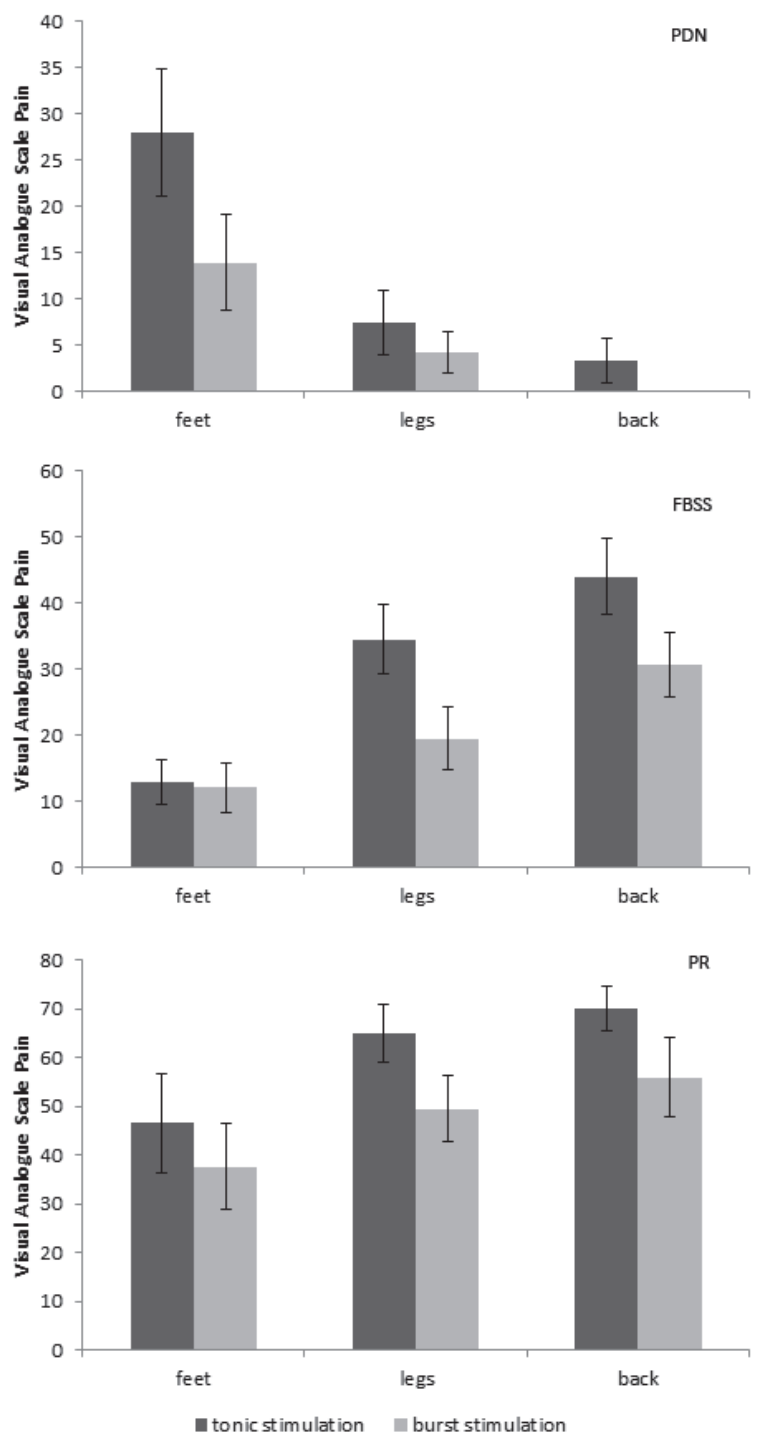

Figure 3. VAS scores for pain the patients with painful diabetic neuropathy (PDN), failed back surgery syndrome (FBSS), and the poor responders (PR) perceived in their feet, legs and back with tonic and burst stimulation. Bars represent the average pain score in a body part, error bars represent standard errors. 
Table 2. Average pain scores at baseline, with tonic and with burst stimulation for the three patients groups.

\begin{tabular}{|c|c|c|c|c|c|c|}
\hline & $\begin{array}{l}\text { baseline } \\
\text { VAS (SD) }\end{array}$ & $\begin{array}{l}\text { tonic } \\
\text { VAS (SD) }\end{array}$ & $\begin{array}{l}\text { burst } \\
\text { VAS (SD) }\end{array}$ & $\begin{array}{l}\mathrm{p} \text {-value } \\
\text { base-tonic }\end{array}$ & $\begin{array}{l}\mathrm{p} \text {-value } \\
\text { base-burst }\end{array}$ & $\begin{array}{l}\mathrm{p} \text {-value } \\
\text { tonic-burst }\end{array}$ \\
\hline \multicolumn{7}{|l|}{ PDN (n=12) } \\
\hline general pain & $70(9)$ & $28(23)$ & $16(18)$ & $<.001$ & $<.001$ & $<.05$ \\
\hline pain feet & & $28(23)$ & $14(18)$ & & & $<.05$ \\
\hline pain legs & & $7(12)$ & $4(8)$ & & & 0.5 \\
\hline pain back & & $3(8)$ & $0(0)$ & & & 0.2 \\
\hline \multicolumn{7}{|l|}{ FBSS $(n=24)$} \\
\hline general pain & $82(7)$ & $49(25)$ & $35(22)$ & $<.001$ & $<.001$ & $<.01$ \\
\hline pain feet & & $13(16)$ & $12(18)$ & & & 0.7 \\
\hline pain legs & & $35(25)$ & $20(22)$ & & & $<.01$ \\
\hline pain back & & $44(28)$ & $31(24)$ & & & $<.05$ \\
\hline \multicolumn{7}{|l|}{$P R(n=12)$} \\
\hline general pain & $82(10)$ & $74(16)$ & $64(27)$ & $<.01$ & $<.05$ & 0.1 \\
\hline pain feet & & $47(35)$ & $38(31)$ & & & 0.1 \\
\hline pain legs & & $65(21)$ & $50(24)$ & & & $<.05$ \\
\hline pain back & & $70(16)$ & $56(28)$ & & & 0.09 \\
\hline
\end{tabular}

PDN (painful diabetic neuropathy), FBSS (failed back surgery syndrome), PR (poor responders)

\section{Failed back surgery syndrome}

The twenty-four patients with FBSS had an average pain score of 82 prior to implantation. With tonic stimulation a significant reduction in comparison to baseline was obtained with an average pain score of 49 . After two weeks of burst stimulation the average pain score was further reduced to 35 with a significant effect in comparison to baseline and tonic stimulation. Fourteen patients (58\%) experienced additional pain reduction with burst stimulation as compared to tonic stimulation; four patients experienced some pain increase.

Patients with FBSS had primarily pain in their legs and back. Forty-six per cent of the patients also had pain in their feet. In this patient group, pain in the feet was generally not altered by burst stimulation in comparison to tonic stimulation, but average pain in the legs and back was reduced by burst stimulation from VAS score 35 to 20 and from 44 to 31 respectively (see Figure 3).

\section{Poor responders}

This group consisted of twelve patients with FBSS who experienced insufficient pain relief with tonic stimulation. They had an average pain score of 82 prior to implantation. After on average two and a half years of tonic stimulation their average pain score was only reduced 
to 74. After two weeks of burst stimulation the average pain score was further reduced to 64 . Six patients (50\%) experienced additional pain reduction with burst stimulation as compared to tonic stimulation, of which three patients experienced a pain reduction of more than $30 \%$. However, one patient experienced a pain increase of $40 \%$.

No significant effect was obtained for this group comparing pain in their feet, which was 46 with tonic stimulation and 38 with burst stimulation. A significant effect was obtained for the legs for burst stimulation (50) in comparison to tonic stimulation (65). A marginal significant difference was obtained for the back when comparing tonic stimulation (70) and burst stimulation (56).

\section{Patient preference}

Tonic stimulation is usually accompanied by paraesthesia, while burst stimulation is aimed to be subthreshold for paraesthesia and should therefore not be sensed by the patients. The perception and appreciation of paraesthesias accompanying tonic stimulation varied to a great extent among the patients (see Table 3), which influenced the patients' preferences for either of the two types of stimulation. Phrases used by patients to describe their experience of the tonic paraesthesias were a. o. annoying, irritating, neutral, part of me, comfortable, pleasant and distraction from the pain.

In contrast to tonic stimulation, there is no possibility yet for patients to adjust the amplitude of burst stimulation themselves. Four patients of the PDN group and ten patients of the FBSS group saw that as a drawback of burst stimulation. These patients generally valued tonic paraesthesia as comfortable or were used to increasing the stimulation amplitude during episodes of increased pain.

Eight patients with PDN and twelve patients with FBSS preferred burst stimulation over tonic stimulation. Six patients from the PR group preferred burst stimulation, even though pain relief obtained with burst stimulation was on average limited. For many patients the absence of paraesthesia is an important advantage of burst stimulation. 
Table 3. Overview of the rating of the perceived tonic paraesthesia, the desire to adjust the burst stimulation amplitude and the stimulation preferences of the number of patients of each group.

\begin{tabular}{lccccccccc}
\hline & \multicolumn{3}{c}{ Tonic paresthesia: } & \multicolumn{2}{c}{ Adjust amplitude: } & \multicolumn{4}{c}{ Preference: } \\
& comfort & neutral & discomfort & yes & no & burst & tonic & either & off \\
\hline PDN & 2 & 8 & 2 & 4 & 8 & 8 & 4 & - & - \\
FBSS & 8 & 9 & 7 & 10 & 14 & 12 & 11 & - & 1 \\
PR & - & 5 & 7 & 2 & 10 & 6 & - & 2 & 4 \\
\hline
\end{tabular}

PDN (painful diabetic neuropathy), FBSS (failed back surgery syndrome), PR (poor responders)

\section{Side effects}

Burst stimulation appeared to be accompanied by side effects, both positive and negative, in patients of all three groups. Negative side effects mentioned by patients were headaches, dizziness and the sensation of 'heavy legs'. Three patients experienced headaches and dizziness; in two patients these side effects sustained during the two week evaluation period, while in the other patient these side effects diminished after a few days. The sensation of heavy legs appeared after half a day of burst stimulation in two patients and continued for the rest of the evaluation period. Positive side effects of burst stimulation mentioned by several patients were the sensation of warm feet and the sensation of peaceful rest in the legs. One patient reported having lower and more stable blood glucose levels during the evaluation period of burst stimulation.

Although burst stimulation should not elicit paraesthesia, several patients did feel paraesthesia when in supine position with burst stimulation at amplitudes that were used. Most of the time the sensation was not experienced as unpleasant, but in a few cases the sensations were perceived as very uncomfortable.

\section{Electrical charge delivery}

The electrical charge per stimulation pulse and the stimulation current were calculated for each patient. Pulse amplitudes used for burst stimulation were lower than for tonic stimulation. Therefore, converting patients from tonic to burst stimulation led to a reduction in the average charge per stimulation pulse from $1.1 \mu \mathrm{C}$ to $0.85 \mu \mathrm{C}(p=.09)$, from $2.8 \mu \mathrm{C}$ to $1.6 \mu \mathrm{C}(p<.01)$ and from $3.2 \mu \mathrm{C}$ to $3.0 \mu \mathrm{C}(p=.81)$ for the PDN, FBSS and PR group respectively. The average stimulation current however rose, from $91 \mu \mathrm{A}$ to $176 \mu \mathrm{A}(p=.09)$, from $177 \mu \mathrm{A}$ to $322 \mu \mathrm{A}(p<.001)$ and from $218 \mu \mathrm{A}$ to $669 \mu \mathrm{A}(p<.05)$ respectively for the same groups, as the number of pulses per second increased when burst stimulation was applied. 


\section{DISCUSSION}

Burst stimulation caused pain reduction in almost all patients. On average burst stimulation caused a significantly larger pain reduction in all three patient groups than tonic stimulation, and burst stimulation caused little or no paraesthesia in most patients. About $60 \%$ of patients (67\% for PDN, 58\% for FBSS and 50\% for the PR group) experienced further pain reduction when applying burst stimulation in comparison to tonic stimulation. An increase in perceived pain as compared to tonic stimulation, starting after one to seven days of burst stimulation, was however mentioned by six patients.

\section{Pain reduction}

In PDN patients the neuropathic pain in their feet and sometimes lower legs already responded very well to tonic stimulation, but burst stimulation reduced the pain even further, in a statistically significant and clinically relevant way. With an average additional pain reduction of $44 \%$ this patient group benefitted relatively most from switching to burst stimulation.

Eleven of the FBSS patients who participated in this study had pain in their feet as well, but burst stimulation was not able to reduce this pain in those patients. This could be due to the position of the electrode lead in the spinal cord, as it was placed to primarily target the lower back and legs. In FBSS patients the tip of the electrode was positioned T7-T10, while in PDN patients the electrode was positioned more caudal: T10-T12. When compared to tonic stimulation, the average pain reducing effect of burst stimulation on leg pain (43\%) is somewhat higher than on back pain (30\%) in FBSS patients. These relative decreases in back and leg pain in this group are comparable with the results of burst stimulation in patients who were naive to SCS [13].

The group of poor responders who had low back and leg pain and over time lost effect of tonic spinal cord stimulation (PR) was a more heterogenic group and consequently had more heterogenic responses as well. Two patients had no preference for either tonic or burst stimulation, four patients rather had their stimulator removed and six patients preferred burst stimulation. Even though the average pain relief was limited in this group, three patients (25\%) did benefit significantly from switching to burst stimulation. 


\section{Paraesthesia}

All patients in this study were familiar with tonic stimulation before they received burst stimulation. They were used to or at least familiar with the paraesthesia. Several patients of both the PDN and the FBSS group also indicated that they actually liked the paraesthesia. The loss or change of the paraesthesia and the inability to increase the stimulation amplitude and thereby the paraesthesia intensity led some patients to prefer tonic stimulation over burst stimulation, in certain cases even despite of the larger pain reduction that was obtained by burst stimulation. In addition, some patients did not perceive the paraesthesia as particularly comfortable, but were able to shift their attention from the pain to the paraesthesia and therefore appreciated it as a distraction from the pain. These patients mentioned that they missed the paraesthesia as a feedback signal that the SCS system is functioning.

In the studies performed by De Ridder et al. $[12,13]$ patients were naive to any form of SCS. The test period of one week of tonic stimulation was probably too short to have patients get acquainted with the paraesthesias accompanying pain reduction. Consequently those patients did not associate pain reduction with paraesthesia and were most likely able to focus primarily on the pain reduction effects of the stimulation paradigms that were tested. As a result all patients preferred burst stimulation.

The majority of the patients in this study however did associate paraesthesia with pain reduction, which complicated the evaluation of the pain reduction effects of burst stimulation. This is possibly due to the fact that paraesthesias reassure the patient the stimulator is active, a prerequisite for conditioned pain relief. Twenty-six (54\%) of all patients in this study preferred burst stimulation.

\section{Stimulation amplitude}

In this study only a two week evaluation was performed, without the possibility to adjust the amplitude during or after the two weeks. This technical inadequacy is important as it can be expected that the efficacy of burst stimulation could be further improved if patients could control the intensity of the stimulation. This could give the patients a feeling of control over their pain, important features in subjective pain perception $[16,17]$.

We have no neurological explanation for the side effects and sensations, both positive and negative, described by some of the patients. Except for the patient who had indeed lower and more stable blood glucose levels during the evaluation period, we were not able to objectify the side effects. However, the side effects must be induced by burst stimulation in 
general or by high amplitudes of burst stimulation, as they disappeared when the patients were converted to tonic stimulation again or when the amplitude was lowered during programming burst stimulation.

It is possible that many of the side effects and paraesthesias could have been reduced or eliminated by lowering the amplitude, while further pain reduction might have been achieved by increasing the stimulation amplitude. A two week evaluation period is however minimally necessary for patients who are already familiar with tonic stimulation. Patients need to get used to the change in sensation, considering the fact that they have been conditioned to associate pain reduction with the presence of paraesthesias. An extended evaluation period that includes the option to adjust stimulation parameters after one or two weeks of stimulation might have been preferable to assess the full possibilities of burst stimulation.

It has been suggested by De Ridder et al. [12] that burst stimulation suppresses pain via the electro-physiological gate-control mechanism before the clinical paresthesia threshold is reached, as the amplitude of effective burst stimulation pulses is lower than the amplitude of effective tonic stimulation pulses. In all three patient groups the charge per pulse in burst stimulation is lower than in conventional tonic stimulation. However, a larger pulse duration and higher pulse frequency in burst stimulation still leads to an increased average stimulation current, which in turn implies an increase in energy consumption and accelerated battery depletion. Future studies should look at intermittent burst stimulation in order to try and decrease energy delivery to the spinal cord. In view of the current calculations and the previously published calculations [12], a 1:2 or 1:3 ratio on:off would be theoretically ideal to exert an energy delivery which is equal to tonic stimulation.

\section{CONCLUSION}

Burst stimulation compares favourably to tonic stimulation for most patients, generally without eliciting paraesthesia. On average burst stimulation causes significantly more pain reduction in patients with painful diabetic neuropathy and in patients with failed back surgery syndrome. A trial period of at least two weeks of burst stimulation for every patient with a spinal cord stimulator would likely increase the efficacy of SCS therapy. Still, further research should elucidate optimal burst stimulation parameters for patients who are already familiar with SCS. 


\section{REFERENCES}

1. de Jongste MJL, Hautvast RWM, Hillege HL, Lie KI. Efficacy of Spinal Cord Stimulation as Adjuvant Therapy for Intractable Angina Pectoris : A Prospective, Randomized Clinical Study. JACC 1994:23;1592-1597.

2. Kemler MA, Barendse GAM, van Kleef M, de Vet HCW, Rijks CPM, Furnee CA, et al. Spinal cord stimulation in patients with chronic reflex sympathetic dystrophy. New Engl J Med 2000:343;618-624.

3. Kumar K, Toth C, Nath RK. Spinal cord stimulation for chronic pain in peripheral neuropathy. Surg Neurol 1996:46;363-369.

4. Kumar K, Taylor RS, Jacques L, Eldabe S, Meglio M, Molet J, et al. Spinal cord stimulation versus conventional medical management for neuropathic pain: A multicentre randomised controlled trial in patients with failed back surgery syndrome. Pain 2007:132;179-188.

5. North RB, Kidd DH, Farrokhi F, Piantadosi SA. Spinal cord stimulation versus repeated lumbosacral spine surgery for chronic pain: a randomized controlled trial. Neurosurgery 2005:56;98-107.

6. Pluijms WA, Slangen R, Joosten EA, Kessels AG, Merkies ISJ, Schaper NC, et al. Electrical spinal cord stimulation in painful diabetic polyneuropathy, a systematic review on treatment efficacy and safety. Eur $J$ Pain 2011:15;783-788.

7. de Vos CC, Rajan V, Steenbergen W, van der Aa HE, Buschman HPJ. Effect and safety of spinal cord stimulation for treatment of chronic pain caused by diabetic neuropathy. J Diabetes Complicat 2009:23;40-45.

8. Nordin M, Nystrom B, Wallin U, Hagbarth KE. Ectopic sensory discharges and paresthesiae in patients with disorders of peripheral nerves, dorsal roots and dorsal columns. Pain 1984:20;231-245.

9. Ochoa JL, Torebjork HE. Paraesthesiae from ectopic impulse generation in human sensory nerves. Brain 1980:103;835-853.

10. van Buyten JP, Al-Kaisy A, Smet I, Palmisani S, Smith T. High-Frequency Spinal Cord Stimulation for the Treatment of Chronic Back Pain Patients: Results of a Prospective Multicenter European Clinical Study. Neuromodulation 2012. doi: 10.1111/ner.12006

11. Tiede J, Brown L, Gekht G, Vallejo R, Yearwood T, Morgan D. Novel spinal cord stimulation parameters in patients with predominant back pain. Neuromodulation 2012. doi:10.1111/ner.12032

12. de Ridder D, Vanneste S, Plazier M, van der Loo E, Menovsky T. Burst Spinal Cord Stimulation: Toward Paresthesia-Free Pain Suppression. Neurosurgery 2010:66;986-990.

13. de Ridder D, Plazier M, Kamerling N, Menovsky T, Vanneste S. Burst Spinal Cord Stimulation for Limb and Back Pain. World Neurosurg 2013. doi: 10.1016/j.wneu.2013.01.040

14. de Vos CC, Dijkstra C, Lenders MWPM, Holsheimer J. Spinal cord stimulation with hybrid lead relieves pain in low back and legs. Neuromodulation 2012:15;118-123

15. Wesselink WA, Holsheimer J, Boom HBK. Analysis of current density and related parameters in spinal cord stimulation. IEEE Trans Rehab Eng 1998:6;200-207

16. Grau JW, Huie JR, Garraway SM, Hook MA, Crown ED, Baumbauer KM et al. Impact of behavioral control on the processing of nociceptive stimulation. Front Physiol 2012: 3; 262

17. Muller MJ. Will it hurt less if I believe I can control it? Influence of actual and perceived control on perceived pain intensity in healthy male individuals: a randomized controlled study. J Behav Med 2012:35;529-537. 



\section{Chapter 7}

\section{Electrode contact configuration and energy}

\section{consumption in spinal cord stimulation}

Cecile C de Vos $^{1}$, Marjolein P Hilgerink ${ }^{2}$, Hendrik PJ Buschman², Jan Holsheimer ${ }^{2}$

1. Department of Neurosurgery, Medisch Spectrum Twente, Enschede, The Netherlands

2. Institute for Biomedical Technology, University of Twente, Enschede, The Netherlands

Neurosurgery 2009; 65 ONS :210-217 


\section{ABSTRACT}

Objective: To test the hypothesis that in spinal cord stimulation, in contrast to an increase of the number of anodes which reduces energy consumption per pulse, an increase of the number of cathodes raises the energy per pulse.

Methods: Patients with an Itrel 3 Pulse Generator and a Pisces Quad quadripolar lead have been selected for this study. A set of 7 standard contact configurations was used in each patient. Resistor network models mimicking these configurations were made. The UT-SCS software has been used to simulate the effect of these contact configurations on large spinal nerve fibres. To allow a comparison of the measured and modelled energy/pulse, all values were normalized.

Results: Both the empirical and the modelling results showed an increase of energy consumption with an increasing number of cathodes. Although the patient data with 1 and 2 cathodes did not differ significantly, energy consumption was significantly higher when 3 cathodes were used instead of 1 cathode or 2 cathodes. The average energy consumption was significantly higher when bipolar stimulation was used instead of monopolar cathodal stimulation. An increasing number of anodes caused a decrease of energy consumption.

Conclusion: When the paraesthesia area can be covered with several configurations, it will be beneficial for the patient to program a configuration with 1 cathode and either no or multiple anodes. 


\section{INTRODUCTION}

Spinal Cord Stimulation (SCS) is a method to relieve chronic, otherwise intractable pain of neuropathic origin. In SCS a lead with multiple contacts is positioned in the epidural space over the dorsal aspect of the spinal cord, a few segments rostral to the level where the nerve roots innervating the painful area enter the spinal cord. The epidural lead is connected subcutaneously to an implanted pulse generator (IPG). Electrical pulses applied by a configuration of cathodal and anodal contacts cause a tingling sensation, called paraesthesia, in the affected part of the body. Ideally, the comfortable paraesthesia covers the pain area completely.

When a battery powered IPG is used, battery lifetime and therefore energy consumption are of great interest for the patient. Depending on the energy needed for pain relief a nonrechargeable battery based IPG has to be replaced within 1 to 7 years $[12,13]$. Although rechargeable IPGs have been introduced recently by manufacturers of SCS equipment, it is still useful to understand the influence of stimulation conditions on energy consumption. According to an FDA safety and effectiveness report, rechargeable IPGs do not need to be replaced as frequently as non-rechargeable batteries, but do have a limited lifespan dependent on energy use [1]. Therefore, energy efficient programming will still benefit the patient.

For a voltage-controlled IPG, the energy required for clinically useful stimulation of a patient is dependent on the voltage and duration of the pulses necessary to evoke the paraesthesia in the patient, the pulse rate and the total impedance between the positive and negative pole. Accordingly, these parameters have to be acquired in order to calculate and compare the energy consumption of various contact configurations. In case a current-controlled IPG is used the same parameters, but current instead of voltage need to be acquired.

In SCS the activation of fibres occurs near a cathode [5]. Therefore, the threshold current to activate fibres is determined by the current injected at the cathode. The total current of the anode(s) is not critical and just has to equalize the total cathodal current. When a single cathode is used in combination with 2 or 3 anodes in parallel, the total anodal current will still be the same as the cathodal (threshold) current. When two or more anodes are used the total anodal impedance will be reduced. Accordingly, the threshold voltage and 
threshold energy will be less. When two or more cathodes are connected in parallel, each cathode needs to inject a (threshold) current. Although the impedance will be reduced, the total current will be increased and the threshold voltage will not be reduced substantially, so that the threshold energy is most likely increased.

The hypothesis tested in this study is that, in contrast to an increase of the number of anodes which reduces energy consumption, an increase of the number of cathodes raises the energy consumption.

In a clinical study we determined the energy per pulse of different SCS contact configurations in 10 patients with an implanted SCS system. In order to determine the effect of the cathodeanode configuration on energy consumption, we determined the maximum tolerable stimulation voltage $\left(\mathrm{V}_{\mathrm{MT}}\right)$ and the total impedance $(\mathrm{Imp})$ of different contact configurations with varying numbers of cathodes and anodes. From these data and the duration of the pulses (PW), which was kept constant for individual patients, the energy/pulse $\left(E_{M T}\right)$ was calculated. In addition, we calculated the energy per pulse while using simple networks of resistors representing contact impedances of the SCS lead and by simulating SCS with the UT-SCS modelling software [4] and calculating the energy/pulse at the discomfort threshold $\left(E_{D T}\right)$.

\section{METHODS}

\section{Materials}

To avoid differences in energy consumption caused by different types of SCS leads and IPGs we included only chronic pain patients with a Pisces Quad lead (model 3487A, Medtronic Inc., Minneapolis, MN, USA) and an Itrel 3 voltage-controlled IPG (model 7425, Medtronic Inc,). The latter has the option to stimulate in a monopolar fashion when the case of the IPG is programmed as anode. The Pisces Quad lead has a rostro-caudal array of 4 contacts at its distal end (figure 1). Each contact can be programmed as an anode (+) or a cathode (-), or can be disconnected (0).

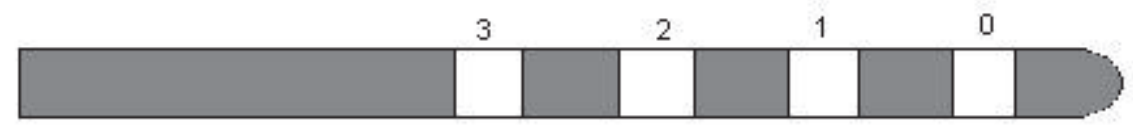

Figure 1. Quadripolar Pisces Quad lead with 4 contacts $(0,1,2,3)$ at its distal end. The cylindrical contacts have a diameter of $\sim 1.3 \mathrm{~mm}$, are $3 \mathrm{~mm}$ long and are separated by $6 \mathrm{~mm}$. 
To program the different contact configurations and acquire information about the energy consumption an N'vision Programmer (Medtronic Inc.) was used. This programmer can be used to measure impedance and current. However, after testing the N'vision Programmer with a resistor of known value, only the impedance measurements turned out to be reliable and therefore these have been used in the analysis.

\section{Contact configurations}

A set of 7 standard contact configurations, presented in table 1, has been used in each patient. Because of anatomical variability, the different contacts of the lead are most likely at different distances from the spinal cord and will thus cause different threshold values [6, 9]. To limit the variation of the output parameters due to this effect, one cathode has been kept at the same lead contact throughout all configurations. The contact configurations were programmed in the same order in all patients.

The 7 standard configurations as shown in table 1 have been tested in any patient who used contact 0 or 1 as a cathode in their personal stimulation settings. When a patient used contact 2 or 3 as a cathode, we measured the same configurations as in table 1, but mirrored, i.e. configurations 1 and 2 would then be: $00-0$ and $0+-+$. This has been done to avoid unfamiliar and uncomfortable paraesthesias in these patients.

Table 1. The standard set of contact configurations selected on a Pisces Quad lead and tested in all 10 patients.

\begin{tabular}{|c|c|c|c|c|c|}
\hline & & \multicolumn{4}{|c|}{ Contact number } \\
\hline & & 0 & 1 & 2 & 3 \\
\hline \multirow[t]{7}{*}{ Configuration } & 1 & 0 & - & 0 & 0 \\
\hline & 2 & + & - & + & 0 \\
\hline & 3 & 0 & - & - & 0 \\
\hline & 4 & + & - & + & + \\
\hline & 5 & 0 & - & + & 0 \\
\hline & 6 & - & - & - & + \\
\hline & 7 & 0 & - & + & + \\
\hline
\end{tabular}

+ indicates that the contact is an anode, - the contact is a cathode and 0 the contact is disconnected 


\section{Empirical study and calculation of energy consumption}

In each patient, the pulse duration (PW) and the pulse rate (PR) were kept constant among all contact configurations, but the PW and PR could be different among patients. For each contact configuration the perception threshold of paraesthesia $\left(V_{\mathrm{PT}}\right)$, the maximum tolerable stimulation voltage $\left(\mathrm{V}_{\mathrm{MT}}\right)$ and the total impedance (Imp) have been determined. $\mathrm{V}_{\mathrm{MT}}$, Imp and PW have been used to calculate the energy/pulse $\left(\mathrm{E}_{\mathrm{MT}}\right)$ for each configuration, according to equation 1

$$
\mathrm{E}_{\mathrm{MT}}=\mathrm{PW} *\left(\mathrm{~V}_{\mathrm{MT}}\right)^{2} / \mathrm{Imp}
$$

If the stimulation current $\mathrm{I}_{\mathrm{MT}}$ is known, $\mathrm{E}_{\mathrm{MT}}$ follows from equation 2

$$
\mathrm{E}_{\mathrm{MT}}=\mathrm{PW} *\left(\mathrm{I}_{\mathrm{MT}}\right)^{2} * \mathrm{Imp}
$$

To allow an accurate measurement of the output parameters, PW and PR have been chosen such that in each patient $\mathrm{V}_{\mathrm{PT}}$ and $\mathrm{V}_{\mathrm{MT}}$ were between 2 and $9 \mathrm{~V}$. Patients were seated in a comfortable chair and postural changes of a patient during a test session were limited as much as possible to avoid variations of perception caused by changing positions of the contacts with respect to the spinal cord. To check whether the body area stimulated by a certain contact configuration differed from the normal paraesthesia felt by the patient, the area of the paraesthesia for each configuration has been indicated by the patient and was noted on a body map. To keep the patients focused on the paraesthesia area, questions about their perception were asked throughout the tests.

In between measurements the stimulator was switched off and the next contact configuration was programmed. To acquire $\mathrm{V}_{\mathrm{PT}}$ and $\mathrm{V}_{\mathrm{MT}}$ for each new configuration, the stimulation amplitude, starting at $0 \mathrm{~V}$, was increased in steps of $0.1 \mathrm{~V}$ until $\mathrm{V}_{\mathrm{PT}}$ and $\mathrm{V}_{\mathrm{MT}}$ were reached.

\section{Resistor network models}

As a first approximation of the effect of varying the contact configuration on $\mathrm{E}$ we mimicked the configurations by simple resistor networks. Each cathode and each anode was represented by a $450 \mathrm{Ohm}$ resistor and included the wire connected to the IPG output. In monopolar cathodal stimulation the resistance of the distant anode was $150 \mathrm{Ohm}$. 
Although systematic studies on SCS electrode impedance are not yet available, it is well known from clinical experience that in bipolar and monopolar stimulation the mean impedance (including lead cable and extension cable) is around $900 \mathrm{Ohm}$ and $600 \mathrm{Ohm}$, respectively. Therefore, a single contact on the lead represents a mean impedance of about $450 \mathrm{Ohm}$. Accordingly, in monopolar stimulation the metal case of the IPG should have an impedance of about $150 \mathrm{Ohm}$. These values have been applied in de resistor network models.

Cathodes were connected in series with anodes, as shown by cathode Rc and anode Ra in figure $2 a$. When a configuration consisted of multiple cathodes and/or anodes, these were respectively connected in parallel, as shown by the anodes Ra1 and Ra2 in figure $2 \mathrm{~b}$. PW was set at $0.2 \mathrm{~ms}$ and the cathodal threshold current for fibre excitation was assumed to be $1 \mathrm{~mA}$. The equations for the equivalent total resistance (Imp) corresponding to monopolar stimulation and tripolar stimulation are given in the legends of figure $2 a$ and $2 b$, respectively. According to equation (2) the energy/ pulse at $\mathrm{MT}\left(\mathrm{E}_{\mathrm{MT}}\right)$ was derived from the calculated equivalent total resistance ( $I \mathrm{mp})$, the selected pulsewidth (PW) and the calculated injected current at MT $\left(\mathrm{I}_{\mathrm{MT}}\right)$ for all contact configurations (table 1 ) and normalized.

A

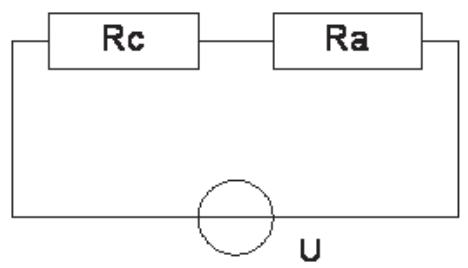

B

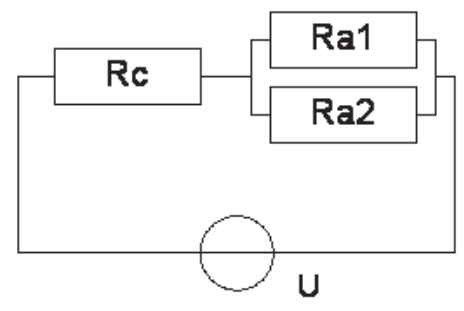

Figure 2. Examples of resistor networks mimicking (a) monopolar stimulation $(0-00)$ with resistance of the cathode ( $\mathrm{Rc}=450 \mathrm{Ohm}$, including resistance of the lead wire) and resistance of the distant anode $(\mathrm{Ra}=150 \mathrm{Ohm})$ connected in series: Imp $R c+R a=600 \mathrm{Ohm}$; (b) tripolar stimulation (+ - + 0) with the resistances of the anodes ( $\mathrm{Ra} 1$ and $\mathrm{Ra} 2$ ) mutually connected in parallel and in series with Rc: Imp $=\frac{(R a 1) \cdot(R a 2)}{(R a 1)+(R a 2)}+R c=525$ Ohm.

\section{Computer Modelling}

The UT-SCS software is a simulation program developed at the University of Twente (The Netherlands) to model the immediate effects of SCS on spinal nerve fibres [4]. The software includes 3D volume conductor models of spinal cord segments with an epidural SCS lead and 
represents the geometry and electrical conductivity of the various anatomical structures in a spinal cord segment, like the grey and white matter of the spinal cord, the surrounding cerebrospinal fluid (CSF), the dura mater, epidural fat and vertebral bone. A Pisces Quad lead was modelled symmetrically in the dorsal epidural space and anode(s) and cathode(s) were set at a positive or negative potential, corresponding to each standard configuration (see table 1).

The average geometry of the 3D model is based on magnetic resonance imaging scans [9] and the electrical conductivities of the various anatomical structures were either taken from literature or calculated from simulation data [11]. As the average thickness of the lowthoracic dorsal CSF layer is 3 - $4 \mathrm{~mm}$, we used the model with a dorsal CSF layer thickness of $3.2 \mathrm{~mm}$. Data about the real thickness of the CSF layer in individual patients was not available, because proper post-implantation CT scans are seldom made.

Furthermore, the software includes electric cable models of large diameter dorsal column (DC) and dorsal root (DR) fibres. These fibres are defined at the anatomically correct places in the 3D models and the field potentials corresponding to their nodes of Ranvier are used as the input of the nerve fibre models. By varying the stimulation voltage between anodes and cathodes the threshold voltage of the lowest threshold DC-fibre and DR-fibre are determined. The perception threshold $\left(\mathrm{V}_{\mathrm{PT}}\right)$ was reached when the membrane voltage of the fibre with the lowest threshold, either the DC fibre or the DR fibre, was depolarized by 70 $\mathrm{mV}$. The discomfort threshold $\left(\mathrm{V}_{\mathrm{DT}}\right)$ was defined as 1.4 times the voltage necessary to excite the lowest threshold DR fibre [3]. In all threshold calculations PW was $0.21 \mathrm{~ms}$.

\section{Data processing and analysis}

The paraesthesia threshold and the discomfort threshold as used in the modelling, are more explicitly defined than the comfort level for a patient, which can be anywhere in between these two. Therefore we have chosen to compare the modelled discomfort thresholds with the maximum tolerable stimulation voltage $\left(\mathrm{V}_{\mathrm{MT}}\right)$ for the individual patient. In the patients, $\mathrm{V}_{\mathrm{MT}}$ is presumably just below the discomfort threshold level. To allow a comparison of the measured and the modelled E values, they were all normalized in the same way. First, for each patient the $\mathrm{E}_{\mathrm{MT}}$ of each contact configuration was normalized to the value of the monopolar configuration (see table 1), which was set at 1.0. Next, the normalized $\mathrm{E}_{\mathrm{MT}}$ values of all configurations with the same number of cathodes or anodes have been averaged for each patient. Then these average values per patient were used to obtain the overall average 
E for the configurations with 1, 2 or 3 cathodes and with 0, 1, 2 or 3 anodes. In all patients the 7 standard configurations from table 1 were acquired.

According to the Shapiro-Wilk test, the patient data is normally distributed. An ANOVA was performed to identify whether there is a significant effect in $\mathrm{E}$. To determine the significance of the differences in the averaged $E$ between configurations with different numbers of cathodes or anodes, a paired T-test was performed and the Holm-Bonferroni method has been used to correct for multiple testing.

\section{RESULTS}

\section{Patients}

Patient characteristics are presented in table 2. Ten patients were included in this study, 3 women and 7 men with an average age of 60 years $(S D=12)$. All had at least 2 years experience with SCS, and a good or very good effect of the stimulation, resulting in an average pain relief rating of $81 \%$ (SD $=9 \%$ ). The majority received SCS for treatment of chronic diabetic neuropathic pain (DNP). One patient suffered from complex regional pain syndrome type I (CRPS) and 2 patients had failed back surgery syndrome (FBSS). One patient had the lead positioned over the cervical segments of the spinal cord, while all other patients had the lead over the lower thoracic segments. The acquired data for the cervically positioned lead do not differ from the thoracic data.

\section{Effect of number of cathodes/anodes on energy consumption}

In figure 3, the energy/pulse of the various contact configurations has been plotted. Figure $3 a$ and $3 b$ show the energy/pulse for contact configurations with an increasing number of cathodes and anodes, respectively. In these figures the values of all measured configurations with the same number of cathodes (figure 3a) and anodes (figure $3 b$ ) are averaged. It is shown that energy consumption increases with an increasing number of cathodes and decreases with an increasing number of anodes (starting from 1 anode) of the SCS contact configuration. Although quantitatively different, both the empirical and the modelled results (resistive networks and SCS models) show a similar tendency. 


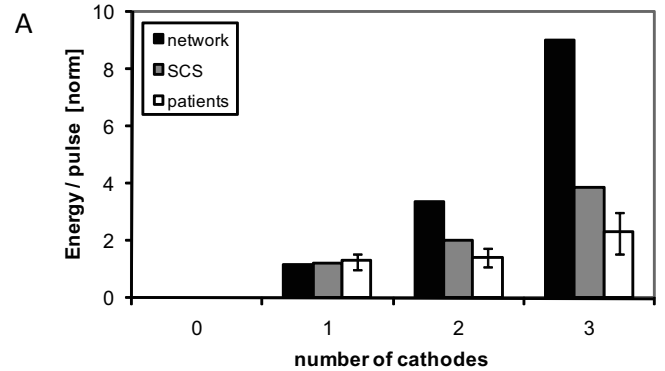

B

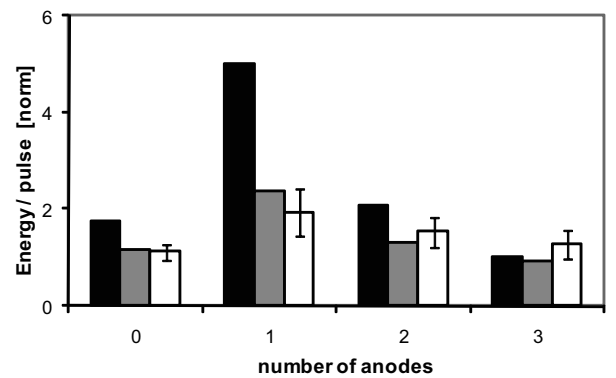

C

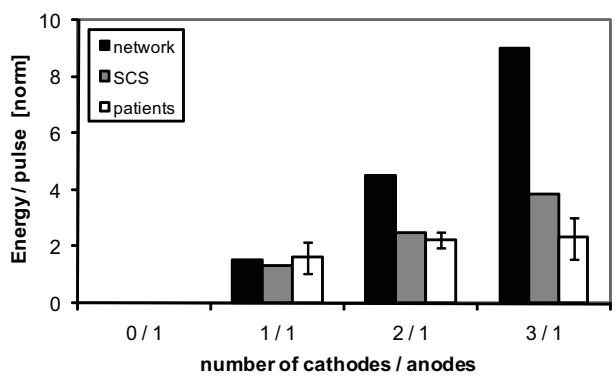

D

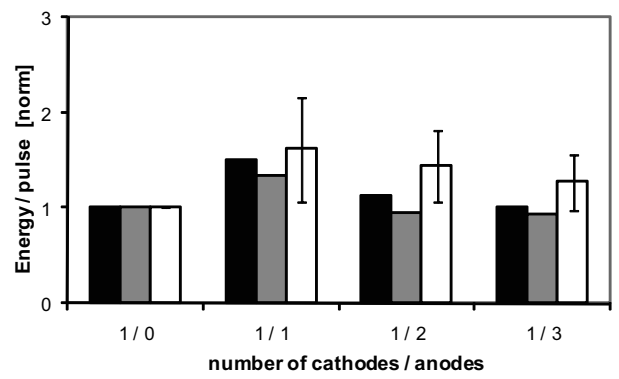

Figure 3. Modelled and empirical E/pulse as a function of the number of cathodes (a) and anodes (b) in the various stimulation configurations. All $E$ values are normalized to the value of the monopolar configuration. The $E$ values of all configurations with the same number of cathodes or anodes are averaged and the empirical data for all 10 patients is averaged as well. In case configurations are not averaged, and the number of anodes is kept constant and only the number of cathodes is varied (c), or when the number of cathodes is kept constant and only the number of anodes is varied (d), a similar pattern is found. 
Table 2. Patient characteristic; pain syndromes include complex regional pain syndrome type I (CRPS), diabetic neuropathic pain (DNP) and failed back surgery syndrome (FBSS.)

\begin{tabular}{ccccccc}
\hline no. & sex & $\begin{array}{c}\text { age } \\
\text { (years) }\end{array}$ & pain syndrome & $\begin{array}{c}\text { position of } \\
\text { cathode(s) }\end{array}$ & $\begin{array}{c}\text { SCS } \\
\text { (years) }\end{array}$ & $\begin{array}{c}\text { Pain relief (\%) } \\
\text { effect of SCS }\end{array}$ \\
\hline 1 & $\mathrm{~F}$ & 43 & CRPS & C3 - C4 & 7 & 90 \\
2 & $\mathrm{~F}$ & 68 & DNP & T9-T10 & 3 & 85 \\
3 & $\mathrm{M}$ & 46 & DNP & T10-T11 & 3 & 80 \\
4 & $\mathrm{M}$ & 73 & DNP & T10-T11 & 3 & 70 \\
5 & $\mathrm{M}$ & 61 & DNP & T10-T11 & 3 & 75 \\
6 & $\mathrm{M}$ & 75 & DNP & T11-T12 & 3 & 65 \\
7 & $\mathrm{~F}$ & 66 & FBSS & T9-T10 & 11 & 80 \\
8 & $\mathrm{M}$ & 49 & FBSS & T9 -T10 & 15 & 90 \\
9 & $\mathrm{M}$ & 73 & DNP & T10-T11 & 2 & 85 \\
10 & $\mathrm{M}$ & 49 & DNP & T10-T11 & 2 & 90 \\
\hline
\end{tabular}

From figure 3a, it is evident that the $E$ values measured in the patients increase less than in both models. Even though the patient data with 1 and 2 cathodes do not differ significantly $(P=0.4), E_{M T}$ is significantly higher when 3 cathodes are used instead of 1 cathode $(P=0.004)$ or 2 cathodes $(P=0.003)$. Figure $3 b$ shows that monopolar stimulation costs least energy. Adding 1 anode increases the energy consumption largely while adding 2 or 3 anodes again reduces the energy/pulse. Just like in figure $3 a$, the modelled results in figure $3 b$ display the effect on $E$ in a better defined way. Nevertheless, the empirical data clearly show the same trend. $E_{M T}$ measured in patients is significantly less $(P=0.001)$ when instead of 1 anode the configuration has no anodes (monopolar cathodal stimulation). Using 2 or 3 anodes instead of 1 anode also results in a significantly lower $E_{M T}(P=0.04$ and 0.001 , respectively). Using 3 instead of 2 anodes causes a significant reduction of $E(P=0.004)$.

Averaging the values for configurations with equal numbers of cathodes and anodes is legitimate, as the same pattern is demonstrated in configurations in which the number of anodes is kept constant and only the number of cathodes is increased (figure 3c). The same is true for configurations where the number of cathodes is kept contact and the number of anodes is increased (figure $3 \mathrm{~d}$ ).

Among the 7 standard configurations those with different numbers of cathodes are not equally distributed. Five combinations have 1 cathode, 1 has 2 and 1 has 3 cathodes. The distribution of configurations with different numbers of anodes is more evenly, as there are 2 configurations without anodes, 2 with 1, 2 with 2 and 1 with 3 anodes. Despite this limitation, the patient outcomes are normally distributed. Moreover, the empirical trends 
are in accordance with the trends predicted by the resistor networks and volume conductor models, although the influence of the number of cathodes or anodes on the energy consumption in patients is less distinct. The variations among the individual patients are relatively small and the $\mathrm{E}_{\mathrm{MT}}$ is better predicted by the $\mathrm{SCS}$ volume conductor model than the resistor network model (table 3 ).

\section{Contact configuration and paraesthesia coverage}

Questioning the patients about the area of the paraesthesia for each configuration revealed that guarding the cathode(s) by adding anodes did not influence the therapeutic range $\left(\mathrm{V}_{\mathrm{DT}} / \mathrm{V}_{\mathrm{PT}}\right)$ or the paraesthesia area in a predictable way. Although two patients hardly experienced any change in paraesthesia attributable to altered contact configurations, most patients mentioned changes in the area where paraesthesia was felt. However, in contrast to the expectations, the areas indicated by the patients were not necessarily larger when anodes were added. Moreover, an increased therapeutic range when stimulation was applied with a 'narrow' bipole instead of monopolarly, as predicted by Law [10], has not been found either. 


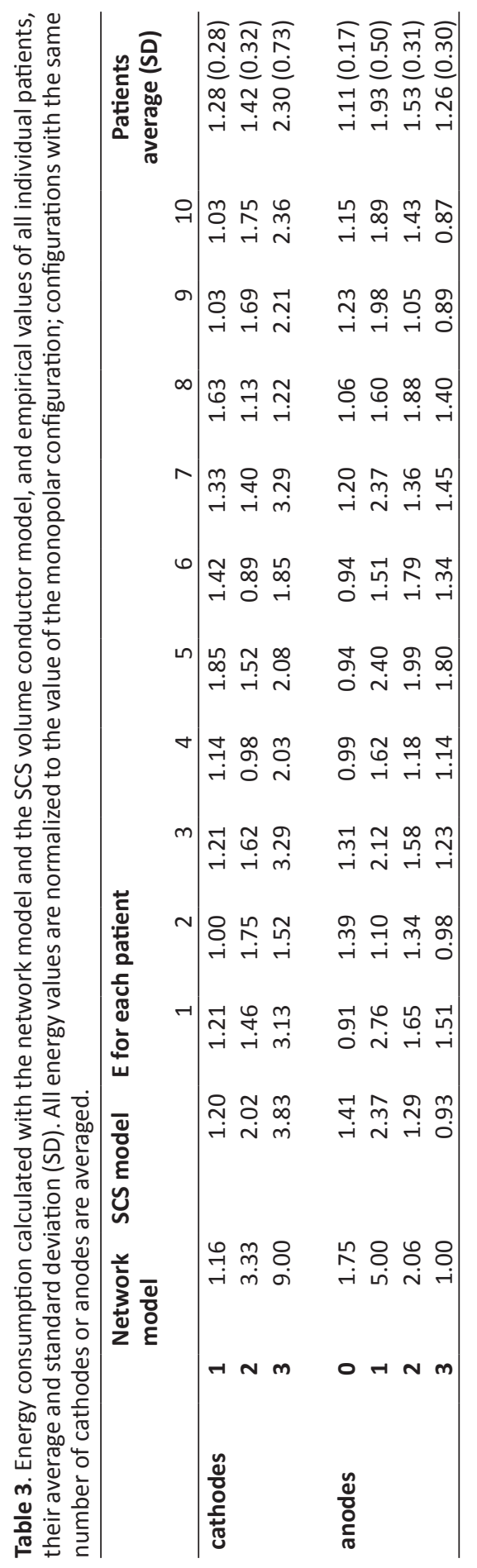




\section{DISCUSSION}

\section{Study hypotheses and validation}

In this study we tested the hypotheses that an increase of the number of cathodes in a contact configuration raises $\mathrm{E}$, whereas an increase of the number of anodes reduces $\mathrm{E}$. The hypotheses have been tested both in a clinical study and theoretically with a simple resistor network and by a realistic 3D volume conductor model. It would be favourable if the outcome of both models would reliably predict the relevant aspects of the empirical results. If so, future analysis could be done by the simple model only.

From the theoretical studies we concluded that $E$ was increased when the number of cathodes was increased from 1 up to 3. Conversely, E was reduced when the number of anodes was increased from 1 up to 3. However, when the number of anodes was further reduced from 1 to zero (monopolar cathodal stimulation) E was reduced as well. These model predictions are in accordance with the results of the empirical study. Despite the small sample size (10 patients) the empirical data show a statistically significant correspondence with the modelled data, except for the difference in E between 1 and 2 cathodes, which was in accordance with the modelled data, but not statistically significant. In 4 patients, the energy required for the 2-cathode configurations was actually lower than for the single cathode. The effect of additional cathodes on E exceeds the effect of additional anodes.

According to the model assumptions the current needed for threshold stimulation $I_{\text {th }}$ with a cathode is constant and the energy/pulse is proportional to $\operatorname{Imp}$ and $\mathrm{I}_{\text {th }}$ (see equation 2). The low energy/pulse in monopolar cathodal stimulation is due to the low Imp of this configuration as compared to bipolar stimulation. The configurations in figure 3 a have 1, 2 or 3 cathodes. For each cathode to be effective, i.e. exciting fibres, it should deliver at least the threshold current. This is shown for $E$ of the network model, which rises more than 7-fold from 1 to 3 cathodes. E calculated from the SCS model and the patients measurements, however, only increased by a factor 3.2 and 1.8, respectively (from 1 to 3 cathodes).

\section{Volume conductor vs. resistor network model}

The substantially smaller increase of the energy/pulse in the patients and the SCS model relates to the $3 \mathrm{D}$ volume conductor properties and the rather small distance of 2 cathodes when connected to adjacent contacts of the SCS lead (see table 1, configuration 3). In the 
SCS computer model each anode or cathode creates a 3D electrical field according to the Poisson equation in the surrounding anatomical structures. When active contacts are far apart these fields do not influence each other, but when contacts are close, they do. As an example, two nearby cathodes will create a stronger field than a single cathode and as a result the required energy/pulse is less than twice the energy needed for a single cathode. This difference was indeed found between the results of the empirical study and SCS modelling study on the one hand and the results of the resistor network model on the other hand. The superposition of cathodal fields and thus the reduction of threshold voltage will be less pronounced when the cathodes are at a larger distance.

The resistor network model has no geometry and obeys Ohms Law (the simple form of the Poissons equation). Each contact is represented by a resistor which is given the same value as the corresponding 3D contact impedance and the same voltages are imposed. Differences in the output parameter energy/pulse due to systematic differences in model parameter values have disappeared by the normalization of the output data. The main difference left between the models is the effect of contact distance, which only exists in the 3D-model. It can be concluded that the resistor network model can be used to predict several features of SCS, but is too simple to predict volume conductor properties.

\section{Clinical aspects}

The present study builds upon that of North et al. [12], who showed that energy consumption is increased when cathodes are added to a contact configuration, but did not analyse the effect when anodes were added. North et al. correctly state that for a given voltage setting additional contacts increase the current drain. However, stimulation of a neural target is primarily related to the injected cathodal current. When an extra contact is added to a configuration, the total impedance will be reduced. In case the additional contact is an anode, the current to obtain a similar clinical effect as before, has to be reduced by reducing the stimulation voltage and thereby the energy consumption. When the additional contact is a cathode the current will be split into two cathodal currents. Because each cathodal current will be less than the initial current, the stimulation current has to be increased by increasing the stimulation voltage and thereby the energy consumption.

Although the empirical measurements were carried out in patients with an Itrel 3 voltagecontrolled IPG which does not deliver a perfect square pulse, like we used in the models, this 
did most probably not affect the results as presented, because both empirical and modelled data were normalized before being compared.

The differences in energy consumption between individual patients are much larger than predicted in the models. This is primarily due to the fact that for example the 3D model has just one set of parameters, but the patients all have different anatomical characteristics. In particular the thickness of the dorsal CSF layer, which varies largely among subjects [6], has a strong influence on $\mathrm{E}$, as well as Imp of the individual lead contacts. Moreover, in contrast to the 3D model, the lead position is not perfectly symmetrical in patients. Although all modelled contacts have identical impedance values, the impedance of the contacts in the patients and thus the corresponding current and $\mathrm{E}$ will generally differ.

Besides the technical limitations, the patient's underlying causes of chronic pain may have altered their perception, giving rise to additional variation in the current needed. In addition, measuring several different configurations can be demanding for the patients, even though, we have used the set of configurations that was most similar to their personal stimulation settings.

\section{Preferred cathode-anode configurations}

Theoretically the paraesthesia area should be larger with a 'narrow' bipole or a guarded cathode than with just a cathode, as these configurations would improve stimulation of the dorsal columns at the expense of dorsal roots $[7,8,15]$. Law [10] has shown empirically that stimulation with a narrowly separated longitudinal bipole was superior to monopolar stimulation and stimulation with a wide bipole, regarding the therapeutic range and paraesthesia coverage in patients with low back pain. North et al. [14] showed a statistically significant preference for a longitudinal guarded cathode. Among all 50 combinations of a 4-pole lead, guarded cathodes were disproportionately preferred by patients with a complex pain syndrome. Instead of the statistically expected $14 \%$ of the 62 patients included in the study, a guarded cathode was selected as the best one by $29 \%$. Although the guarded cathode most probably provides the largest paraesthesia coverage, a majority of $71 \%$ still prefers another configuration, most likely providing an improved coverage of the pain area with paraesthesias. Stimulation with a 'narrow' guarded cathode or a 'narrow' bipole is probably a good initial guess, although the optimal configuration for an individual patient will generally be slightly different. 
In our empirical study, guarding the cathode(s) did not influence the paraesthesia area in a predictable way, which is in accordance with the stochastic nature of this relation. Although most patients mentioned changes in the area where paraesthesia was felt, the area indicated by a patient was not necessarily larger when anodes were added. In some cases the altered paraesthesia areas even caused an uncomfortable or undesirable sensation.

Although adding anodes will reduce energy consumption, therapeutically it is not always favourable for the individual patient. According to North et al. a trade-off between therapeutic effect and energy saving has to be made. When little difference in therapeutic effect exists between two contact configurations, but when one is substantially more energy saving, it may be beneficial for the patient to use the configuration that is second best therapeutically [12].

Programming interleaving pulse trains increases the flexibility in paraesthesia coverage strongly and at the same time increases energy consumption because the total number of pulses per second is increased. However, this SCS method has not been incorporated in our study, primarily because the IPG we used (Itrel 3) does not allow this method.

When stimulation is applied with dual leads with 8 contacts each, the number of active contacts (anodes and cathodes) is usually not substantially larger than with a single quadripolar lead. Alo et al. [2] analysed the contact configurations in 62 patients with dual leads and reported a mean of 5 contacts ( 2 cathodes and 3 anodes). In $40 \%$ of the patients the best configuration was 1 or 2 bipoles and in $48 \% 1$ or 2 guarded cathodes on adjacent contacts of both leads. Therefore, it is expected that the results of our study are also valid for dual lead stimulation with 16 contacts.

The hypotheses that an increase of the number of cathodes in SCS raises the energy consumption and that using more than 1 anode reduces the energy consumption have been confirmed by both the empirical study and the theoretical studies. This implies that when the paresthesia area can be covered with several configurations, it can be beneficial for the patient to program a configuration with only 1 cathode and either none or several anodes.

\section{Acknowledgement}

The authors would like to acknowledge Medtronic for an unrestricted research grant. 


\section{REFERENCES}

1. PRECISION ${ }^{\mathrm{TM}}$ Spinal Cord Stimulation (SCS) System - P030017, U.S. Food and Drug Administration, department of Health and Human Services, http://www.fda.gov/cdrh/PDF3/p030017b.pdf, accessed on 12/05/07

2. Alo KM, Redko V, Charnov J: Four year follow-up of dual electrode spinal cord stimulation for chronic pain. Neuromodulation 5: 79-88, 2002

3. Holsheimer J: Effectiveness of spinal cord stimulation in the management of chronic pain: analysis of technical drawbacks and solutions. Neurosurgery 40: 990-996, 1997

4. Holsheimer J: Computer modelling of spinal cord stimulation and its contribution to therapeutic efficacy (review). Spinal Cord 36: 531- 540, 1998

5. Holsheimer J: Which Neuronal Elements are Activated Directly by Spinal Cord Stimulation. Neuromodulation 5: 25-31, 2002

6. Holsheimer J, Barolat G: Spinal geometry and paresthesia coverage in spinal cord stimulation. Neuromodulation 1: 129-136, 1998

7. Holsheimer J, Wesselink WA: Effect of anode-cathode configuration on paresthesia coverage in spinal cord stimulation. Neurosurgery 41: 654- 660, 1997

8. Holsheimer J, Wesselink WA: Optimum electrode geometry for spinal cord stimulation: the narrow bipole and tripole. Medical \& Biological Engineering \& Computing 35: 493-497, 1997

9. Holsheimer J, Den Boer JA, Struijk JJ, Rozeboom AR: MR assessment of the normal position of the spinal cord in the spinal canal. AJNR Am J Neuroradiol 15: 951-959, 1994.

10 Law JD: Spinal stimulation: statistical superiority of monophasic stimulation of narrowly separated, longitudinal bipoles having rostral cathodes. Applied Neurophysiology 46:129-37, 1983

11. Manola L, Holsheimer J, Veltink P: Technical performance of percutaneous leads for spinal cod stimulation; a modelling study. Neuromodulation 8: 88-99, 2005

12. North RB, Brigham DD, Khalessie A, Calkins SK, Piantadosi S, Campbell DS, Daly MJ, Dey PB, Barolat G and Taylor R: Spinal cord stimulator adjustments to maximize implanted battery longevity; a randomized controlled trial using a computerized patient-interactive programmer. Neuromodulation 7: 13-25, 2004

13. North RB, Calkins SK, Campbell DS, Sieracki JM, Piantadosi S, Daly MJ, Dey PB, Barolat G: Automated, patient-interactive, spinal cord stimulator adjustment: A randomized controlled trial. Neurosurgery 52: $572-$ 579,2003

14. North RB, Ewend MG, Lawton MT, Piantadosi S: Spinal cord stimulation for chronic, intractable pain: superiority of "multi-channel" devices. Pain 44: 119-130, 1991

15. Oakley JC, Prager JP: Spinal cord stimulation mechanisms of action. Spine 27: 2574-5283, 2002 


\section{Chapter 8}

\section{Pain evoked potentials in chronic pain patients}

\section{with spinal cord stimulation}

Cecile C de $\operatorname{Vos}^{1,2,3,4}$, Marjanne J Bom³, Michel JAM van Putten ${ }^{2,3}$

1. Department of Neurosurgery, Medisch Spectrum Twente, Enschede, the Netherlands

2. Department of Clinical Neurophysiology, Medisch Spectrum Twente, Enschede, the Netherlands

3. MIRA biomedical technology and technical medicine, CNPH group, University of Twente, the Netherlands

4. the Neurobionics Foundation, Enschede, the Netherlands 


\section{ABSTRACT}

Objective: This pilot study is an explorative study to investigate whether alterations in the cortical processing of somatosensory stimuli correlate with the efficacy of burst spinal cord stimulation (SCS).

Methods: Twenty patients with chronic pain and fourteen control subjects without pain participated. All patients had pain in their legs, back and/or feet due to failed back surgery syndrome or diabetic neuropathy and were treated with SCS. Intra-epidermal electrical stimulation was applied at the right dorsum of the hand when burst SCS was on and off. Evoked potentials were recorded over the contralateral sensory cortex.

Results: Fourteen patients experienced over 30\% pain reduction with burst SCS and were defined responders. Responders to burst SCS showed a decrease in P200 amplitude of the contralateral cortical evoked potential when stimulation was on compared to stimulation off. This modulation appeared absent in non-responders. The level of the reported pain reduction due to burst stimulation is correlated with the decrease in P200 amplitude.

Conclusion: The magnitude of pain relief by spinal cord stimulation seems to be reflected in the P200 amplitude of the pain evoked potential. 


\section{INTRODUCTION}

Chronic pain interferes significantly with the quality of life and general functioning of about $18 \%$ of the population of Western European countries people and is accompanied by large socioeconomic costs [Wolff 2011, Eriksen 2003]. When treatment with analgesic medication is not successful, spinal cord stimulation (SCS) might be an option. SCS has shown to be an effective treatment for various neuropathic pain conditions including failed back surgery syndrome (FBSS) [North 2005; Kumar 2007], complex regional pain syndrome (CRPS) [Kemler 2000], angina pectoris [De Jongste 1994; Mannheimer 1998] and painful diabetic neuropathy [Tesfaye 1996, De Vos 2009].

Spinal cord stimulation is an invasive treatment for chronic pain, based on electrical stimulation of the dorsal columns of the spinal cord [Simpson 2003]. To target the nerves fibres in the dorsal column, an electrode lead is surgically implanted in the epidural space and connected subcutaneously to an implanted pulse generator. The electrical stimulation of the large myelinated $A \beta$ fibres generally causes reduction of the perceived pain and at the same time elicits paraesthesia in most patients. Paraesthesia is usually described as a tingling sensation perceived in same the body area(s) as the pain was perceived.

Burst stimulation is a new spinal cord stimulation paradigm, administering bursts of electrical pulses with a frequency of $500 \mathrm{~Hz}$. This stimulation mode was designed to induce pain reduction without eliciting the paraesthesias that accompany conventional spinal cord stimulation [De Ridder 2010; De Ridder 2013]. This novel stimulation paradigm demonstrated to improve pain reduction in about $60 \%$ of the patients who already have conventional, tonic spinal cord stimulation for at least six months [De Vos 2013].

Imaging studies have demonstrated that chronic pain can alter brain functioning by structural remapping and functional reorganization of various brain areas and circuits [Kupers 2006, Tracey 2008, Saab 2012]. Imaging studies using positron emission tomography (PET) [Kishima 2010] and functional magnetic resonance imaging (fMRI) [Stancak 2008, Moens 2012] have also shown that SCS can alter the activity in a large number of brain regions. The role and importance of alterations in each region have not been elucidated yet. 
Alterations of brain functioning because of chronic pain or SCS could result in modulation of the cortical processing of sensory stimuli, in particular painful stimuli. Many chronic pain patients suffer from allodynia or hyperalgesia. To activate and study the nociceptive system various stimuli can be used, but ideally Adelta or C fibres are activated selectively. Laser stimulation, eliciting a laser evoked potential (LEP), is capable of selective activation, but has some limitations as the time required to heat the skin causes a delay between stimulus application and activation of the nociceptors and there is a risk of burning the skin. Several researchers have suggested the use of intra-epidermal electrical stimulation (IES) as a method of selectively activating nociceptors [Bromm 1984, Inui 2002]. Mouraux and co-workers have demonstrated that Adelta nociceptors can indeed be selectively activated with IES [Mouraux 2010].

Previously we studied the differences in clinical efficacy between conventional tonic spinal cord stimulation and burst spinal cord stimulation [De Vos 2013]. In this pilot study, we explore if cortically evoked potentials elicited by IES in patients treated with burst stimulation reflect changes in the effectiveness of the pain reduction.

\section{METHODS}

\section{Subjects}

Intra-epidermal electrical stimulation evoked potentials (IES-EP) were measured in 14 healthy subjects without pain and 20 patients with SCS because of chronic neuropathic pain in the lower extremities. All patients had SCS for at least 6 months and the SCS electrode was positioned in the epidural space between thoracic levels T7 - T10, to target the lower extremities and back. The study conformed to the Declaration of Helsinki, was approved by the institutional review board of Medisch Spectrum Twente and all patients gave informed consent prior to participation.

\section{Intra-epidermal electrical stimulation (IES)}

We applied IES via a concentric bipolar needle electrode designed by Inui et al. [2002]. The electrode was positioned on the dorsum of the right hand, which was not affected and a non-painful body area for all subjects. 
Thirty electrical pulses of $500 \mu$ s duration were applied. The inter stimulus interval was randomly varied between 3.5 - $10 \mathrm{~s}$. For al subjects and in both conditions the stimulation amplitude was four times the sensation threshold, determined for every patient individually. During the measurements, patients were asked to silently count the number of applied pulses and rated the IES sensation prior and after the measurement on a visual analogue scale (VAS: from 0 representing no pain to 100 worst possible pain).

\section{Evoked Potential recording and Data analysis}

Continuous EEG was acquired at $5 \mathrm{kHz}$ during the application of IES using a 64-channel EEG setup (TMS-international and ANT software). Evoked potentials were evaluated at C5-Fz and Cz-M1M2, only. Data analysis was performed offline using Matlab (the Mathworks inc, MA, USA).

The EEG data was down sampled to $1 \mathrm{kHz}$ and filtered with $2^{\text {nd }}$ order bandpass filter $0.5-45$ $\mathrm{Hz}$. The data was segmented into epochs from $-200 \mathrm{~ms}$ to $500 \mathrm{~ms}$ and a baseline correction was performed using the 200 ms pre stimulus interval. Muscular artefacts were manually removed, ICA was used to correct for eye movements.

As recommended by $\mathrm{Hu}$ et al. [2010] we used C5 referred to $\mathrm{Fz}$ (C5-Fz) to assess the contralateral IES-EP: N150 and P200. Cz referred to linked mastoid electrodes (Cz-M1M2) was used to assess the vertex IES-EP: P100, N200, P300. Cruccu et al. [2008] recommended to use $\mathrm{Cz}$-nose, but this was not possible with our EEG set up.

\section{Experimental protocol}

Each control subject had one IES-EP measurement and each patient was measured twice: once with burst stimulation and once with no stimulation. The twenty SCS patients who participated in this study evaluated burst spinal cord stimulation for two weeks. The EP measurement during burst stimulation was done at the end of the two weeks evaluation period. The 'off' measurement was done one month after the evaluation period, and patients were asked to switch off their stimulation for at least 12 hours prior to the measurement. Scores for perceived on-going pain (VAS) were assessed at the beginning of each measurement. Responders were defined as those patients who had at least $30 \%$ pain reduction with burst stimulation as compared to no stimulation. 


\section{Statistics}

Paired t-tests were performed for both responders and non-responders, to compare latencies and amplitudes of the N150, P200 (measured at the contralateral cortex) and P100, N200 and P300 (measured at the vertex) in the off-condition with the burst-condition.

\section{RESULTS}

\section{Subjects}

Fourteen healthy control subjects without pain participated. From the twenty patients who participated, fourteen patients were responders to burst stimulation and six patients were non-responders. All patients had pain in their legs, feet and/or back, none of the control subjects had pain (Table 1). None of the subjects experienced the IES as painful. Some of the patients who were in pain had difficulties to concentrate on the IES, however all subjects were asked to count the applied IES and all counted them correctly (within 5\% accuracy).

Table 1. Subject characteristics, average intra epidermal stimulation amplitude and pain scores

\begin{tabular}{lccccccccc}
\hline & & & \multicolumn{3}{c}{ Pain (VAS) } & \multicolumn{3}{c}{ IES Amplitude (mA) } & \multicolumn{2}{c}{ IES Pain (VAS) } \\
& sex $(\mathbf{m} / \mathbf{f})$ & age $(\mathbf{y})$ & scS $(\mathbf{y})$ & burst & off & burst & off & Burst & off \\
\hline responders & $6 / 8$ & 57 & 2.9 & 22 & 63 & 5.7 & 4.7 & 7 & 8 \\
non-responders & $3 / 3$ & 47 & 1.7 & 63 & 64 & 2.1 & 2.1 & 13 & 16 \\
control subjects & $7 / 7$ & 41 & n.a. & n.a. & 0 & n.a. & 2.0 & n.a. & 12 \\
\hline
\end{tabular}

\section{Evoked Potentials}

Evoked potentials at the contralateral somatosensory cortex ( $\mathrm{C} 5-\mathrm{Fz})$ for both responders and non-responders to burst stimulation are shown in Figure 1. With burst stimulation on, the responders had an average P200 amplitude that resembled the P200 amplitude of the control subjects. 


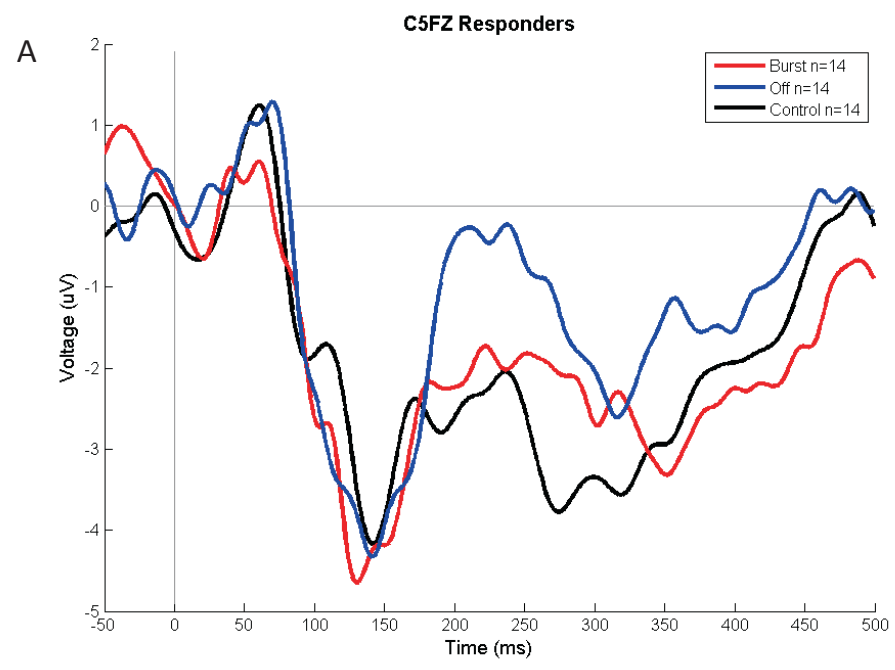

B

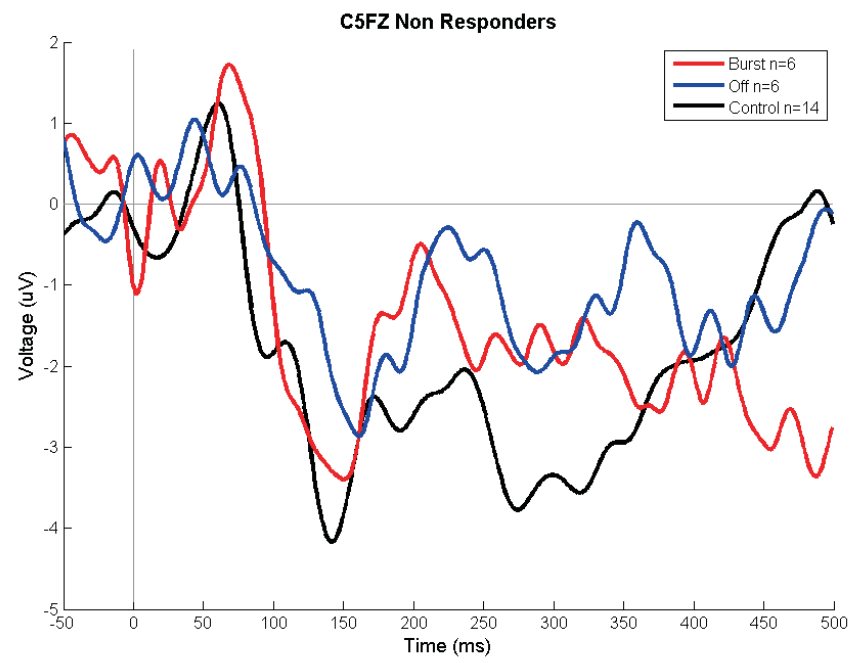

Figure 1. (a) Average contralateral IES evoked potentials for responders $(n=14)$ with burst stimulation off (blue curve) and on (red) compared to the healthy controls ( $n=14$, black). There is a significant reduction in the P200 amplitude ( $p=0.02)$. (b) Average contralateral IES evoked potentials for the nonresponders $(n=6)$, with absence of significant amplitude reduction.

The non-responders to burst stimulation $(n=6)$ experienced pain in both conditions: burst stimulation on and off. There is on average no difference in P200 amplitude between the two conditions for the non-responders. Although not very strong, there is a correlation $\left(R^{2}=0.33\right)$ between the effect of burst stimulation the individual patients report and their decrease in P200 amplitude (Figure 2). 


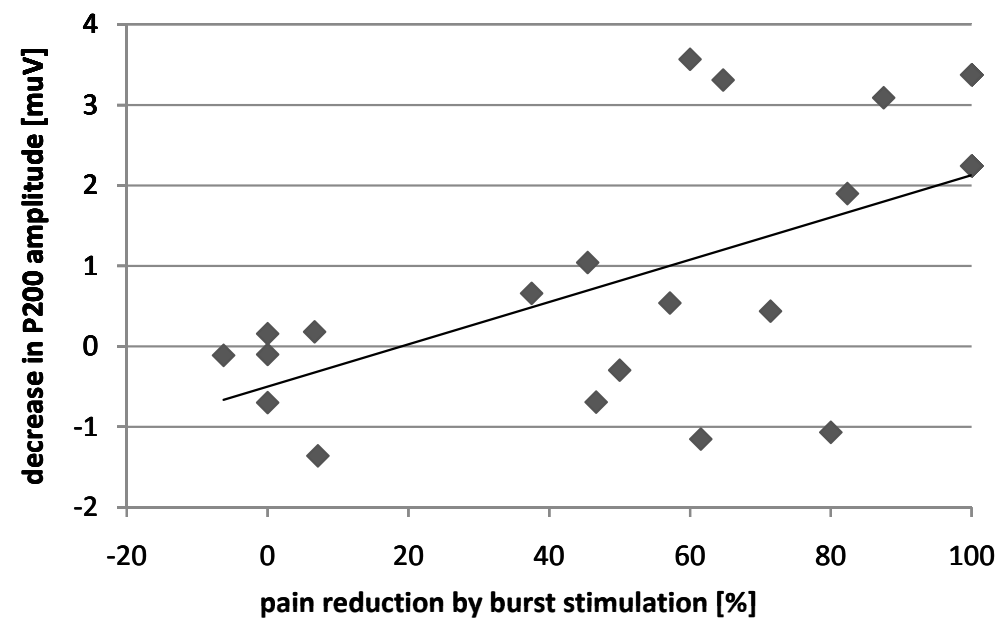

Figure 2. The correlation between the decrease in P200 amplitude (in $\mu \mathrm{V}$ ) and the pain reduction (in percentage) the individual patients obtained with burst stimulation compared with no stimulation. $\mathrm{R}^{2}=0.33$.

IES evoked potentials at the vertex (Cz-M1M2) for both responders and non-responders to burst spinal cord stimulation are shown in Figure 3. Statistical significant differences between the two conditions were found for neither responders nor non-responders. There was also no statistical significant difference between control subjects and patients. 

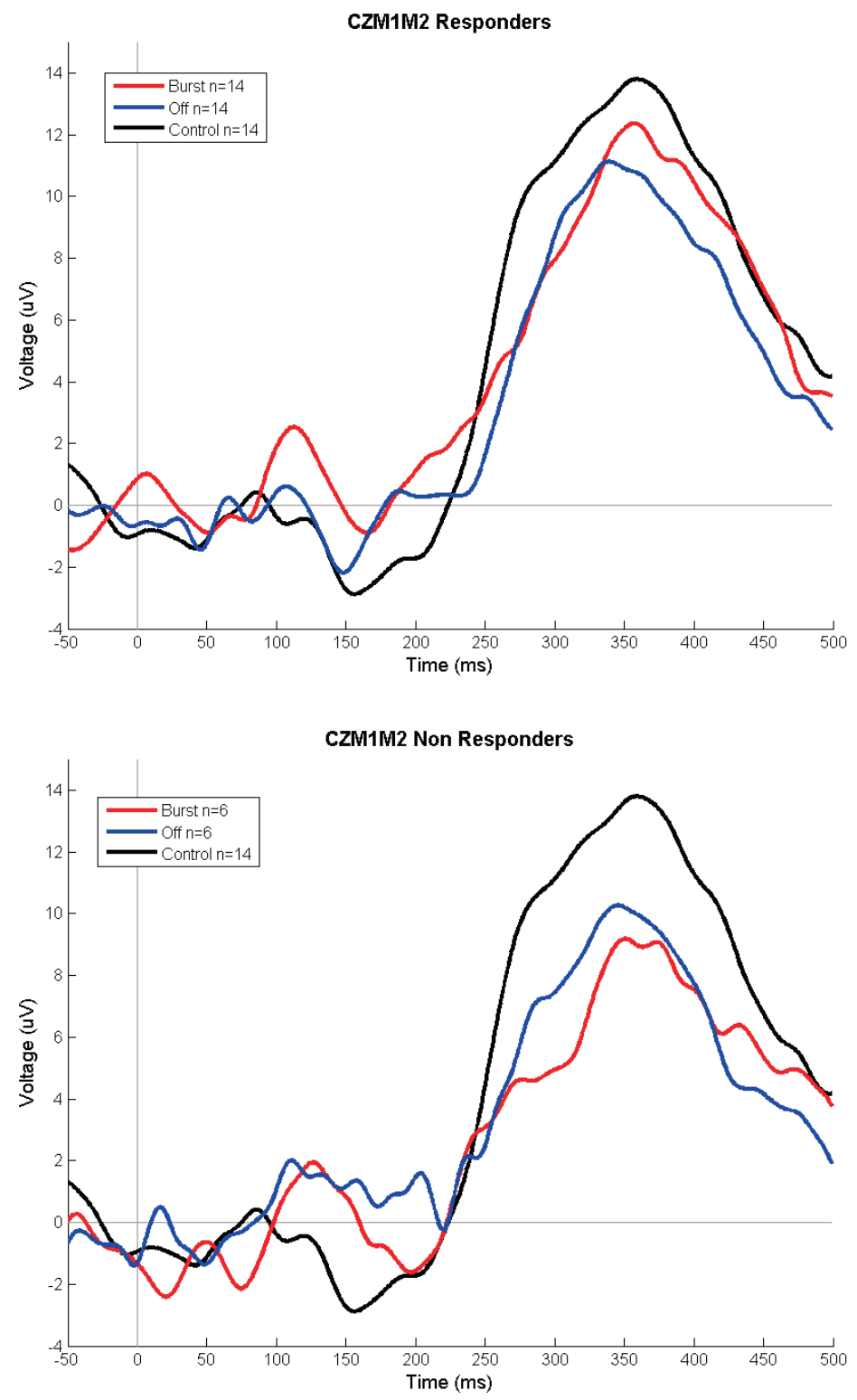

Figure 3. (a) Vertex IES evoked potentials for responders ( $n=14)$ with burst stimulation off (blue curve) and on (red) compared to the healthy controls (black), (b) Vertex IES evoked potentials for the nonresponders $(n=6)$. There is no significant amplitude modulation. 
Table 2. Average latencies (in ms) and amplitudes (in muV) of IES evoked potentials in responders and non-responders to spinal cord stimulation, $\mathrm{p}$-values of the paired t-tests in the patient groups.

\begin{tabular}{lcrrrrrr}
\hline & $\begin{array}{l}\text { Responders } \\
\text { (n=14) }\end{array}$ & & \multicolumn{3}{c}{$\begin{array}{l}\text { Non-responders } \\
(\mathbf{n}=6)\end{array}$} & $\begin{array}{r}\text { Controls } \\
\text { (n=14) }\end{array}$ \\
\hline C5-Fz & burst & off & p-value & \multicolumn{1}{c}{ burst } & off & p-value & \\
lat N150 & 139,2 & 144,9 & 0,4 & 148,7 & 158,4 & 0,4 & 144,4 \\
lat P200 & 212,3 & 216,2 & 0,7 & 195,6 & 213,6 & 0,4 & 206,2 \\
N150 & $-5,3$ & $-5,5$ & 0,7 & $-4,6$ & $-3,9$ & 0,7 & $-5,7$ \\
P200 & $-0,1$ & 1,1 & 0,02 & 0,0 & 0,9 & 0,9 & $-0,1$ \\
N150-P200 & 5,1 & 6,6 & 0,06 & 4,6 & 4,8 & 0,7 & 5,6 \\
Cz-M1M2 & & & & & & & \\
lat P100 & 119,8 & 117,8 & 0,5 & 113,1 & 116,2 & 0,6 & 110,7 \\
lat N200 & 213,1 & 225,2 & 0,08 & 206,0 & 222,3 & 0,3 & 210,4 \\
lat P300 & 348,8 & 342,0 & 0,6 & 351,7 & 351,7 & 1 & 337,2 \\
P100 & 4,1 & 2,2 & 0,06 & 3,0 & 2,8 & 0,9 & 2,4 \\
N200 & $-2,1$ & $-2,3$ & 0,8 & $-3,2$ & $-3,5$ & 0,9 & $-4,6$ \\
P300 & 15,2 & 13,1 & 0,2 & 11,5 & 11,3 & 0,9 & 16,3 \\
N200/P300 & 17,3 & 15,4 & 0,3 & 14,8 & 14,8 & 1 & 20,9 \\
\hline
\end{tabular}

\section{DISCUSSION}

In this pilot study, we found that modulation of the P200 amplitude, as recorded at the contralateral somatosensory cortex, is correlated with the change in pain perception in patients treated with burst spinal cord stimulation. The amplitude of the P200 was increased when patients had the stimulation off and experienced ongoing pain. However, when patients were successfully treated with burst stimulation (responders), their average P200 amplitude decreased significantly and during burst stimulation their P200 amplitude resembled the P200 from healthy controls. Non-responders to burst stimulation did not show this amplitude decrease when the stimulation was on.

Our results show modulation of the P200 elicited by IES also when a non-painful body part is stimulated; indicating indeed that "being in pain" causes central changes in sensory processing.

Being in pain seems to make the brain areas generating the P200 more susceptible for processing additional noxious stimulation. However, this does not influence the subjective rating of the applied intra-epidermal electrical stimulus. 
For every patient the IES amplitude was four times the individually determined sensation threshold. Objectively the responders had a higher stimulation amplitude, because on average they had a higher sensation threshold than the controls and the non-responders. For both patient groups there was no difference between the mean stimulation thresholds for the burst condition and the off condition. Patients and control subjects rated the stimulation pulses as non-painful, reflected in VAS scores below 20 for all conditions. So, subjectively every patient received comparable IES.

The N150-P200 complex is believed to originate from the insula, secondary somatosensory and cingulate cortices [Bromm 1998, Inui 2003] and its peak-to-peak amplitude has been related to subjective ratings of stimulus intensity and pain. Both the N150 and the P200 are, therefore, thought to reflect cognitive-evaluative processes involved in stimulus perception. We did not find differences in subjective ratings of the intensity of the applied intra-epidermal electrical stimulus itself. The only difference between the two conditions patients reported was the intensity of their ongoing pain.

Roosink et al [Roosink 2011] applied IES and found no differences in P200 and N150-P200 peak-to-peak amplitudes between the affected and unaffected side in patients with post stroke shoulder pain. All these patients however had ongoing pain. This confirms our finding that experiencing pain in general influences the modulation of the contralateral P200 amplitude regardless of location of the applied stimuli.

According to Valeriani et al. [2006] in the nociceptive system, the cortical reorganisation involves only the representation of the painful body part, while the adjacent cortical areas are unmodified. They applied laser stimulation at the hand and face of healthy subjects while only their hand was hurt, as a result only modulation in the hand-LEP was found. The LEP N200/P300 amplitudes recorded on the vertex decreased when a painful body area was stimulated. We did not stimulate the painful body area of the patients with spinal cord stimulation, which could explain why we did not find any significant modulations of the vertex potential.

Another possible important difference is the studied population: we measured in patients with chronic, primarily neuropathic pain and Valeriani et al. measured in healthy subjects who received short-lasting nociceptive pain. 
They only other study we could find that reported pain evoked potential measurements in patients with SCS was done by Sestito et al. [2008] who measured LEPs in patients with SCS for the treatment of cardiac syndrome X. They did find a decreased vertex N200/P300 amplitude when SCS was off compared to when SCS was on, but only when they stimulated the painful chest of the patients, not when they stimulated the pain-free hand. They did not evaluate the contralateral cortical evoked potential.

The number of subjects in this explorative study is small, particularly the number of nonresponders to burst stimulation. This limits the relevance of statistical evaluations. However, the correlation between the magnitude of pain reduction caused by burst stimulation and the modulation of the P200 suggests that the intensity of the generally perceived ongoing pain enhances cortical processing of sensory stimuli.

Pain evoked potentials, whether elicit by laser stimulation or intra-epidermal electrical stimulation, exhibit large interpersonal differences, this is particularly the case in patients with neuropathic, central pain [Gacia-Larrea 2002]. Latencies can vary to a great extent and therefore complicate averaging EPs from a group of patients. Figures 1 and 3 depict the average EPs of the responders and the non-responders. Because of differences in latency between the individual patients, these average EPs are not very smooth and differ from the values in table 2. Paired t-tests were however performed on the peaks of the EPs of the individual patients.

In conclusion, the magnitude of pain relief obtained by spinal cord stimulation seems to be reflected in the reduction of the P200 amplitude of the pain evoked potential. However, a larger population with a greater variety in responses to spinal cord stimulation needs to be studied, preferably combined with imaging and source localisation techniques. This may provide more details about the localisation and extent of the presumed cortical networks involved in pain processing in patients with chronic pain. 


\section{REFERENCES}

Bromm B, Meier W. The intracutaneous stimulus: a new pain model for algesimetric studies. Methods Find Exp Clin Pharmacol 1984; 6: 405-410

Bromm B, Lorenz J, Neurophysiological evaluation of pain. Electroencephalography and clinical Neurophysiology 1998; 107: 227-253

Cruccu et al. Recommendations for the clinical use of somatosensory-evoked potentials. Clinical Neurophysiology 2008; 119: 1705-1719

De Jongste MJ, Hautvast RW, Hillege HL, Lie KI. Efficacy of spinal cord stimulation as adjuvant therapy for intractable angina pectoris: a prospective, randomized clinical study. Working Group on Neurocardiology. J Am Coll Cardiol 1994; 23: 1592-1597

De Ridder D, Vanneste S, Plazier M, van der Loo E, Menovsky T. Burst Spinal Cord Stimulation: Toward ParesthesiaFree Pain Suppression. Neurosurgery 2010: 66; 986-990

De Ridder D, Plazier M, Kamerling N, Menovsky T, Vanneste S. Burst Spinal Cord Stimulation for Limb and Back Pain. World Neurosurg 2013. doi: 10.1016/j.wneu.2013.01.040

De Vos CC, Rajan V, Steenbergen W, van der Aa HE, Buschman HPJ. Effect and safety of spinal cord stimulation for treatment of chronic pain caused by diabetic neuropathy. Journal of diabetes and its complications 2009; 23: $40-45$

De Vos CC, Bom MJ, Vanneste S, Lenders MWPM, de Ridder D, Burst spinal cord stimulation evaluated in patients with failed back surgery syndrome and painful diabetic neuropathy, Neuromodulation, 2013

Garcia-Larrea L et al. Laser-evoked potential abnormalities in central pain patients: the influence of spontaneous and provoked pain. Brain 2002; 12: 2766-2781

Hu L, Mouraux A, Hu Y, lannetti GD, A novel approach for enhancing the signal-to-noise ratio and detecting automatically event-related potentials (ERPs) in single trials, Neurolmage 2010; 50: 99-111

Inui K, Tran TD, Hoshiyama M, Kakigi R. Preferential stimulation of Adelta fibers by intra-epidermal needle electrode in humans. Pain 2002; 96: 247-52

Inui K, A comparative magnetoencephalographic study of cortical activations evoked by noxious and innocuous somatosensory stimulations, Neuroscience 2003; 120: 235-248

Kemler MA, Barendse GAM, van Kleef M, de Vet HCW, Rijks CPM, Furnee CA, van den Wildenberg FAJM, Spinal cord stimulation in patients with chronic reflex sympathetic dystrophy, N Engl J Med 2000; 343: 618-24

Kumar K, Taylor RS, Jacques L et al. Spinal cord stimulation versus conventional medical management for neuropathic pain: a multicentre randomised controlled trial in patients with failed back surgery syndrome. Pain 2007; 132: 179-188

Mannheimer C, Eliasson T, Augustinsson LE et al. Electrical stimulation versus coronary artery bypass surgery in severe angina pectoris: the ESBY study. Circulation 1998; 97: 1157-1163

Mouraux A, GD lannetti, L Plaghki, Low intensity intra-epidermal electrical stimulation can activate Ad-nociceptors selectively. Pain 2010; 150: 199-207

North RB, Kidd DH, Farrokhi F, Piantadosi SA. Spinal cord stimulation versus repeated lumbosacral spine surgery for chronic pain: a randomized, controlled trial. Neurosurgery 2005; 56: 98-106

Sestito A, Lanza GA, Le Pera D, De Armas L, Sgueglia GA, Infusino F, Miliucci R, Tonali PA, Crea F, Valeriani M. Spinal cord stimulation normalizes abnormal cortical pain processing in patients with cardiac syndrome X. Pain 2008; 139: 82-89

Simpson BA, editor. Electrical stimulation and the relief of pain. Pain research and clinical management vol.15, Amsterdam: Elsevier; 2003

Smits $\mathrm{H}$, van Kleef M, Holsheimer J, Joosten EAJ. Experimental Spinal Cord Stimulation and Neuropathic Pain: Mechanism of Action, Technical Aspects, and Effectiveness. Pain Practice 2013; 13: 154-168

Tesfaye S, Watt J, Benbow SJ, Pang KA, Miles J, MacFarlane IA. Electrical spinal cord stimulation for painful diabetic peripheral neuropathy. Lancet 1996; 348: 1698-1701

Valeriani $\mathrm{M}$ et al. Modulation of laser-evoked potentials by experimental cutaneous tonic pain. Neuroscience 2006; 140: $1301-1310$ 



\section{Chapter 9}

\section{Living with Spinal Cord Stimulation}

Lucie Dalibert ${ }^{1}$, Cecile C de Vos ${ }^{2,3}$

1. Department of Philosophy, University of Twente, Enschede, The Netherlands

2. Department of Clinical Neurophysiology, University of Twente, the Netherlands

3. Department of Neurosurgery, Medisch Spectrum Twente, the Netherlands

Shortened version of "Living and Composing with a Somatechnology", submitted to Science, Technology, \& Human Values 


\section{ABSTRACT}

Spinal cord stimulation (SCS) is an implanted neuromodulation technology resorted to in the treatment of chronic pain. Some philosophical frameworks have been developed to understand human-technology relations and the mediating effects of technology. However, they have been limited to technologies situated in use-configurations that are outside the body. Others, in the postphenomenological tradition, have attempted to conceptualise implanted technologies such as cochlear implants, but these frameworks are still insufficient to understand what is happening with SCS. Based on fieldwork, this article proposes to apprehend SCS as a somatechnology. When patients with chronic pain enter into intimate relationships with the technology, the remaking of bodies becomes crucial. SCS constitutes a re-worlding for patients living with the device, it requires an active incorporation process through which new bodies are enacted - materialised. The body-self is recomposed. The intimacy that characterises the relation between patients living with SCS and their device also constitutes an unfolding of relations, the quality of which is pivotal for successful lives with SCS. These reflections are nonetheless not limited to philosophical considerations as professionals, like physicians and biomedical engineers, can benefit from apprehending SCS technology as a somatechnology. 


\section{INTRODUCTION}

In the last decade, technologies such as implants have received quite a lot of attention by philosophers of technology. Especially if they touch upon neurological functions, they have been seen as having profound implications for what it means to be human [McGee 2008; Verbeek 2009, 2011]. Yet, reflections on these technologies have generally taken the shape of (rather speculative) psychological and ethical judgments on 'hyped' technological devices, like brain computer interfaces and deep brain stimulation [e.g. Sandberg 2006; Vedder 2010; Nordmann 2007; Woopen 2012]. However, no or scarce attention has been paid to the most common neuromodulation technology: Spinal Cord Stimulation (SCS), a technology to treat chronic pain. Philosophy of technology would benefit from an exploration of the implications of and experiences with SCS, as it falls short of frameworks for understanding what is at stake with this relatively new technology. What it means to live with such a device, can shed new light on human-technology relations and renew the philosophical frameworks that have attempted to conceptualise them.

The importance of renewing philosophical frameworks for apprehending human-technology relations and implications of entering an incorporation process is not limited to the field of philosophy of technology alone. Professionals working with neuromodulation technologies like physicians and biomedical engineers need to understand the consequences of living with SCS. They are responsible for referencing eligible pain patients, implanting neuromodulation systems, counselling the patients throughout the entire process and developing new neuromodulation technologies. Each new development can affect or alter the process of embodiment and the patient's experiences with SCS.

First, we will briefly introduce the neuromodulation device used for SCS. Second, we will discuss philosophical frameworks for conceptualising human-technology relations. The current approach to technical mediation and intentionality will appear to be limited to apprehend SCS. Third, we will show, based on interviews with patients and professionals, that living with SCS requires active incorporation and composition processes. Conceptualising these processes will be necessary for renewing current philosophical frameworks. 


\section{SPINAL CORD STIMULATION}

\section{Neuromodulation, Pain and Paraesthesia}

A SCS system is a type of neuromodulation device that is used as a last resort treatment of chronic neuropathic pain. Chronic pain is probably the most common neurological disorder and in many cases it is difficult to treat it with medication. Intractable chronic pain severely impairs people's lives as it has a negative influence on almost all aspects of life: on one's physical abilities, sleep, social life, mental health and wellbeing - on one's world [Breivik 2006; Jackson 1994].

A neuromodulation device is invasive technology that acts directly upon neural tissue. It consists of the modulation of neural activity through the delivery of electrical energy directly to a target area. It works by stimulating specific brain areas or nerves to produce biological responses that might have been disturbed or diminished because of a disease or a medical condition.

What SCS is believed to do is interrupting or reducing the signals sent from the painful body part towards the brain. In most cases, pain is replaced by another sensation: paraesthesia. Paraesthesia is by many patients described as a tingling sensation. The perception and appreciation of paraesthesia is very diverse, varying from pleasant to annoying. In most cases however, paraesthesia is very bearable or hardly noticed after a while.

The stimulation settings of SCS can be adjusted to the patient's preference or need. Stimulation frequency is one of the parameters that can be adapted, and for many patients it largely influences the perceived paraesthesia. Stimulation with frequencies below $30 \mathrm{~Hz}$ evokes more distinct tingling sensations described as many tiny prickles/tickles, whereas stimulation with higher frequencies is generally experienced as a smoother sensation. New stimulation paradigms with intermittent or continuous stimulation frequencies of $500 \mathrm{~Hz}$ and above are believed not to cause any paraesthesia at all and to achieve good results as well [De Ridder 2010, 2013; Van Buyten 2012]. Of the fifteen patients living with SCS with whom we conducted semi-structured interviews, fourteen had just been part of a two weeks trial with one of these new stimulation paradigms.

\section{Implantation and interaction}

The implantation of SCS device comprises two phases. First, the electrode lead is implanted in the epidural space and connected to a temporary pulse generator outside the body. There 
is a consecutive trial period of several days. Only if the trial period is successful, that is, if a patient experiences significant pain relief, the external pulse generator is converted into an implanted pulse generator. This pulse generator is implanted under the skin of the lower abdomen or upper buttock and the pulse generator can be quite heavy and rather bulky. In the Netherlands, most of the implanted pulse generators are still non-rechargeable and have to be surgically replaced after three to seven years. During the implantation of the electrode lead in epidural space, the patient who has received local anaesthesia is lying prone on the operating table and must be awake to provide information on whether the dorsal column is stimulated at the right side and level: the paraesthesia elicited by the stimulation needs to cover the painful area. Many patients experience the implantation of the electrode as a demanding procedure.

The implanted parts are however not the only pieces of technology that constitute the SCS system. There is also an external part: a remote control enables the patients to interact with the implanted device and adjust the stimulation settings. Adjustments of the settings however, take place within predefined limits. The physician or nurse, in coordination with the patient and his or her needs, sets one or more stimulation programmes, as well as the upper and lower limits of the stimulation amplitude in the implanted pulse generator. Different amplitudes or programmes can be used when pain varies during the day, as a result of different activities or postures. An augmentation of the stimulation amplitude causes an increase in the sensation of paraesthesia. Usually this increases the pain suppression effect or distracts the patient from the pain, although (too) high stimulation amplitudes can be perceived as very uncomfortable and even painful.

\section{SPINAL CORD STIMULATION AND TECHNICAL MEDIATION}

\section{Technologies as Instances of Mediation}

Phenomenology recognises that humans do not have a direct, but rather a mediated, relation with the world. Our lifeworld is permeated with and co-constituted by technologies. As such, one is not directly in bodily-sensory experience present to the world, but rather via a technological artefact, such as when one wears glasses, watches television, or uses a mobile phone [Verbeek 2005]. Postphenomenology has made the concept of 'technical mediation' central in its understanding of the ways in which technologies affect, modify, and co-constitute one's actions, perceptions, and experiences of ourselves and the world, 
as well as morality [e.g. Ihde 1979, 1990; Verbeek 2005, 2006, 2011]. Unfortunately, even though postphenomenology seems to constitute a promising framework for understanding what is at stake with SCS [Lettow 2011], attention to any implanted technology in general has been scarce [Ihde 2008, De Preester 2011].

In his postphenomenological framework, Don Ihde identifies two instances of technical mediation: hermeneutic and embodiment relations. In hermeneutic relations, the world is perceived by means of an artefact that provides an interpretation of the world (e.g. a thermometer or a radio-telescope). In embodiment relations, the world is experienced through an artefact that is perceived as a 'quasi me' (e.g. a dentist probe or spectacles). Notwithstanding the value and usefulness of Ihde's typology of relations, and more generally of the concept of technical mediation, 'mediation theory' becomes ill-suited when technologies entering in ever more intimate relations with human beings. Technologies such as SCS are no longer in a 'use configuration', but rather merge with the body and reconfigure it.

\section{Cyborg relations and hybrid intentionalities}

In this respect, Peter-Paul Verbeek has drawn attention to the fact that with implanted technologies, mediation gives way to (physical) merger. Cyborg relations replace embodiment relations, and technologically mediated intentionality - i.e. directedness towards the world - becomes hybrid intentionality. '[T]here actually is no association of a human and a technology anymore. Rather, a new entity comes about' [Verbeek 2008, p.391]. In addition to the cyborg relation and its correlate hybrid intentionality, Verbeek identifies another human-technology configuration that goes beyond technical mediation. Composite intentionality refines Ihde's hermeneutic relation and is formed out of the combination of technological intentionality and human intentionality. With his exploration of hybrid and composite intentionality, Verbeek attempts to bring postphenomenological frameworks beyond use configurations.

Yet, despite the heuristic value of cyborg relation and hybrid intentionality in drawing attention to the specificity of implanted technologies (compared to usable technological artefacts), the terms do not explicate the practicalities, workings, or underpinnings of these novel human-technology configurations. Assuredly, the concept of hybrid intentionality highlights the intertwinement of humans, world, and technology, and thereby acknowledges the human's 'cyborg ontology' [Haraway 1991]. Nevertheless, this raises 
the question of hybrid intentionality being ultimately a matter of interiority and invisibility of the technological device? With Verbeek's postphenomenological framework, hybrid intentionality becomes performative: implanted technologies are expected to dramatically transform one's intentionality, so much so that 'a new entity comes about.' Furthermore, while technology and the human physically merge within the cyborg relation, human bodies and their re-shaping with/in technologies are silenced. As Kirk Besmer examines how cochlear implants $(\mathrm{Cl})$ are embodied by their users, his analysis attempts to remedy these shortcomings [Besmer 2012]. As such, it is a particularly interesting starting point for understanding what is at stake with SCS.

\section{Implanted technologies and the importance of bodies in technologies}

As Besmer relies upon personal stories and academic reports about the experiences of people living with $\mathrm{Cl}$, he shows how living with the implanted device entails re-exploring and re-conceptualising existing phenomenological frameworks. More particularly, drawing upon work on technical mediation of perception [Ihde 1979, 1990] and Verbeek's subsequent additions [Verbeek 2008, 2011], Besmer highlights that exploring the experiences of $\mathrm{Cl}$ wearers compels philosophical analyses to focus on embodiment. In the case of the $\mathrm{Cl}$, the human-technology relation involves both an active embodiment process and a degree of merger between the implant and its wearer. The relation between the $\mathrm{Cl}$ and its wearer engenders a specific type of embodiment and intentionality, both 'cyborg.' They respectively share elements of Ihde's embodiment and hermeneutic relations and of Verbeek's hybrid and composite intentionalities, yet in both cases they are reducible to neither [Besmer 2012, p.311].

Living satisfactorily or successfully with a $\mathrm{Cl}$ necessitates an active, intense, and extensive learning (and embodiment) process. Similarly to the SCS system, the $\mathrm{Cl}$ is composed of different devices or components, some internal, other external. Cochlear implants do not amplify sound as hearing aids do but rather stimulate electronically the auditory nerve fibres, whereby auditory signals can be received and interpreted as sound. For the electronic stimulation to become meaningful auditory information, however, the process is not straightforward. The implantation procedure is only the first step of a lengthy and demanding rehabilitation journey. $\mathrm{Cl}$ wearers, like patients with SCS, undergo mapping sessions, during which the implant is fine-tuned so as to set the optimal stimulation programme(s) for each individual. 
As Besmer shows, if the $\mathrm{Cl}$ and its wearer merge, this is not so much a physical merger, but one in which the algorithm of the speech processor translates the environmental sounds into a series of electrical pulses on the auditory nerve which are meaningful signals to the $\mathrm{Cl}$ wearer. Due to the coded algorithm, the $\mathrm{Cl}$ and one's nervous system merge as electronic and nerve signals interflow. The intentionality is a hybrid perceptual intentionality. Undeniably, cochlear implants dramatically change one's perception and being-in-the-world, from a world of silence to a world of sound and noise. In this re-worlding, in which $\mathrm{Cl}$ wearers describe their experience as 'coming back to life'. Their rehabilitation is like a transformation, rather than recovery.

The resonance with the experiences of patients living with SCS is remarkable. Both during the implantation of the electrode and subsequent mapping sessions, the stimulation programme(s) and amplitude(s) are adjusted. As an electrical field that matches the body's painful areas is mapped onto the spinal cord, the nervous system merges with the stimulation of the pulse generator. The pain recedes or is replaced by paraesthesia. Although 'a new world emerges around a new body' constituting a 'complete re-worlding,' the re-worlding is of a different nature with SCS than with a $\mathrm{Cl}$.

Successfully hearing with a $\mathrm{Cl}$ and exhibiting a 'cyborg intentionality' is the outcome of an extended learning process, one in which embodiment is pivotal. Although embodiment is also essential with respect to SCS, it is quite different for both implanted technologies. According to Besmer, 'for the $\mathrm{Cl}$ to function, the hermeneutic relation must become embodied' (p.309). Indeed, if the Cl eventually becomes an extension of one's perceptual body and withdrawn from one's attention (i.e. becomes quasi-transparent), which are two features of Ihde's embodiment relation, it entails an experience of hearing that is rather different, from a technologically unmediated perception (p.306). Cl wearers must undertake and go through 'rehabilitation,' during which they must learn how to discriminate between noise and meaningful sound, as well as create 'auditory memories.' In other words, they must (re-)learn how to hear (p.304).

\section{SCS AS SOMATECHNOLOGY}

Although Besmer's postphenomenological analysis of hearing with a $\mathrm{Cl}$ sheds some light on the experience of living with SCS, some substantial differences exist between the two implanted technologies. Besmer attempted to enrich mediation theory by making 
intentionality its focal point. Yet, the emphasis on intentionality and the conception of the body as a perceptual entity do not allow for accurately apprehending what is at stake with SCS. If the intimate relationship taking place between patients and the SCS technology is to be understood, both people and the technological device in their materiality have to be accounted for. Technological artefacts are recognised as being agential in postphenomenological frameworks, while human bodies are denied such agency. However, it is our contention that in order to account for active implants, we must attend to the making or materialisation of bodies with/in SCS.

\section{Somatechnologies}

SCS exhibits technological intentionality. Yet, the implanted device is not oriented towards the world, as understood by the postphenomenological frameworks devised by Ihde, Verbeek and Besmer, but towards the human body. The implanted technology is directed towards one's pain. As the spinal cord is stimulated, the burning or stabbing pain felt in one's body part is reduced and replaced by paraesthesia. In pain and in paraesthesia, the body cannot be conceived as mere intermediary, as becoming as one with the implanted technology without being transformed. Another body materialises with SCS. As such, the implanted technology must be apprehended as a somatechnology.

While Susanne Lettow understands somatechnologies as 'all those technologies intentionally geared toward modifying bodies' [Lettow 2011, p.110], she proposed this concept to draw attention to philosophy of technology's neglect of bodies and urge the field to stop 'referring to an anthropological essence of "the human" [and instead] analyse, in an integral way, the various contingent and stratified bodily technological practices' (p.116). As a technology that acts on and intervenes in bodies, SCS is a somatechnology. Bodies are both transformed and enacted with the implanted technology. As SCS affects one's posture and movements, it reshapes one's body, but it also enacts the body's multiple ontology, while bodies reconfigure the implanted technology.

\section{SCS and the remaking of the body}

In the rehabilitation process embodiment of SCS and habituation to new sensations e.g. paraesthesia and reduced pain - postures and movements are pivotal. Even spinal cord stimulation's scripted behaviour, namely the interaction with the neuromodulation technology via the remote control, is corporeal. The remote control - or the presence 
thereof - steers one's action and behaviour towards resorting to it, rather than to one's bodily postures and movements, for adjusting the stimulation. Assuredly, the realisation or actualisation of that material invitation might eventually become routinized, ritualised and settled in one's practices, and become part of one's repertoire of bodily techniques. In one's relations with SCS, the remote control is, nevertheless, not necessarily central. The stories told by the patients we interviewed testify to the fact that some never use the remote control and others interact with or steer the stimulation by using their bodies.

"The feeling goes on through the legs and back here [..] I feel it. And then, if I am laying down, or if I pull my head back, I feel it very clearly [... If I have pain in my legs, I do like this [she pushes her head backwards] then it vibrates very strongly for a moment and then the pain decreases".

It is through her postures and movements that Mrs A directs the stimulation. In this way SCS not only effectuates - puts in motion - her body, but also creates bodily sensations - the feeling of paraesthesia progressively replacing the pain. The neuromodulation technology realises and redefines her body. In Ihde's embodiment relation and his example of experiencing a blackboard with a fine dentist probe or one's finger, movement is also primordial as there can be no touch or sensation thereof, without movement. Movements are the practices through which one's becoming with SCS takes place. Through the enactment of certain movements and the correlated effect and affect of the stimulation, patients start living with SCS.

"The first, two, three days, yes, yes all day you're just playing with it. Yes. What is possible? Can I drive the car? ... When you're sitting down and you put it on: oh, that's very nice. And then you go stand up: iuuuuuuuuuh! Yes? ... You put it higher: aaah, it's not possible! And then you must learn: higher: what can you do? Yes? I put it on, and I try, I feel this [..] or a little lower ... [W] hen I have a lot of pain, it goes higher. And when I lay down, I put my arm up, you can djjjuuuu! And my ears go a bit [laughing and scratching his ears] You cannot lay down, and you must put it lower...It stimulates much more and then your toes are buh-dutdut: you put it too high".

While Mr B's account illustrates the difficulties that exist in talking about one's body and sensations, it exemplifies the active process of becoming with technology. Through the 
enactment of movements, SCS becomes incorporated. When experimenting with SCS, a patient is moved by the implanted technology. New movements are performed, thereby enacting a different body. Many technological artefacts have such a corporeal dimension, however SCS technology is distinct insofar as not only bodily movements are created or transformed, but also one's bodily sensations are reconfigured. A different body materialises with SCS.

$\mathrm{Cl}$ wearers experience a re-worlding that is due to the practical implications of the implanted technology (being able to partake in some activities). In the case of patients living with SCS, a re-worlding also takes place. This re-worlding is linked to the neuromodulation technology's practical dimensions, such as finally being able to walk, but not exclusively. Unlike the $\mathrm{Cl}$, the re-worlding does not stem only from perception being reconfigured, but also from the materialisation of one's own body with technology.

\section{Materialising bodies with/in SCS}

Pain - the body in pain and the painful body - is pivotal for understanding the re-worlding that patients living with SCS experience. The implanted technology is a last resort treatment of chronic pain. Therefore, prior to having the neuromodulation device implanted, everyone has experienced various, and generally heavy, types of analgesic medication. Patients living with SCS do not lack words neither to express the sensation of pain nor the perception of one's heavily medicated self. Mr C, for instance, recalls that he stopped taking medication

"[b]ecause yes, my mind was flat, so to speak. And I did not noticed that so well, but my wife and the people around me who noticed said it, and that is an unpleasant experience to hear afterwards [..] So then, I said resolutely: I quit, and that is what we did. And ... then I read a study [..] with respect to neurostimulation [..] And that was an investigation [... .... And then I called them to go and I was actually invited to participate in that study. ... Look [..] that treatment is aimed for pain suppression, so yes, you also have that hope that it will happen ... and it has also helped some. But pff [..] that they measure pain on a scale of 1 to 10 ... that works for me, but pain is quite subjective of course, but if I say my pain is about an 8 , then what they can do for you is scale it in a sense, what your pain experience would be ... [S]o they measure that a bit. Yes and actually my pain went from 7.5 / 8 to 7, but the pain that I have in my feet is constant [..] I'm always in southern France along the river, on the pebbles on my bare feet [..] so you should imagine walking on it. But, well, I also have a lot of flashy shooting pain. In my feet and toes. And often I have cold feet. And for these last two aspects, there the stimulator is working nicely. That's pretty much reduced." 
Mr C's graphic recounting of his experience with medication and living with pain is exemplary of the materialisation of bodies with SCS. When he describes his experience with pain as living one's life as if constantly walking bare feet on pebbles and being continuously 'shot' by pain, $\mathrm{Mr} C$ shows how pain is not only 'unquestionably a sensation in a part or parts of the body, but also always unpleasant and therefore an emotional experience,' and therefore incarnates the definition of pain used by the International Association for the Study of Pain [IASP]. Pain is multidimensional. Measuring or assessing whether or not someone experiences chronic pain is based on primarily subjective criteria. One puts words on the painful sensation, thereby making it communicable and measureable via an analogous pain rating scale ranging from zero to ten. However, as Isabelle Baszanger points out, in each medical encounter, 'the evaluation and legitimation of pain - the question of credibility arises because pain is a sensation that can be directly perceived only by the person who feels it' [Baszanger 1989, p.427].

SCS interacts with the nervous system and enacts the body. As the paraesthesia covers and replaces the painful sensation, the body-in-pain is effectuated alongside the body-inparaesthesia (the body without, or at least in less, pain). The body is multiple, to borrow Annemarie Mol's expression [Mol 2002]. The ontology of one's body is neither one nor determined. As such, SCS is beyond technical mediation: the technology does not only transform one's movements and kinaesthetic experience, but it enacts one's embodied self which materialises with the implanted technology.

\section{Re-composing oneself with SCS}

Living with SCS, therefore, involves a re-worlding. When sensing pain and feeling paraesthesia, the body becomes multiple. Besides, for people living with/in pain, "body and self seem different' [Charmaz 1999, p.364]. Furthermore, as Mr C reminded us: with analgesic medication, he was flat. As bodies become multiple, one's sense of embodied self is regained. Both pain and pain medication, because of the ways they affect and effect the body and sense of self, are experienced as intruders. In fact, the materialisation of the bodyin-pain, the body-in-paraesthesia, with the body with/in SCS constitute a breaking point for (post)phenomenology. Chronic pain is not an intentional object; it does not belong to one's field of perception but is rather an overwhelming and all-pervasive sensation that constrains one's field of possibilities [Scarry 1985]. Being a body-in-pain, enacts a certain world, in which one's possibilities are hindered and hampered. This world is experienced as 
shrivelled. Within this state of being-in-the-world, one might feel that one's body and self are incongruous. As Mr C illustrates, however, not only the body-in-pain but also the bodyin-pain-medication can enact a disagreement between one's felt body and sense of self. One becomes a stranger to oneself. When the body 'learns (how) to be affected' by SCS, the subject is being recomposed.

Experimenting with the technology, the ability to steer the stimulation, hence the level of paraesthesia and pain, through one's postures or with the help of the remote control, all contribute to re-articulate the body-self. As new stimulation paradigms emerge for SCS and cause pain reduction without paraesthesia, the ways in which SCS is incorporated and the ways in which the body-self is being re-articulated will most likely be altered. Most of the patients we interviewed experienced this new stimulation paradigm for two weeks. This redefined the experiences with SCS and the kind of bodies that materialise with/in the technology as well as the ways in which the self is recomposed. $\mathrm{Mr}$ C for instance, recalls that the SCS system becomes a part of your life very quickly:

"It's just that the tingling feeling .. if that is gone .. the last 14 days I did not have the tingling sensation and one does get used to that again as well .. But the first few days it's like going outside without a coat - like you miss a part of your body."

The body-self learns to be affected. When recomposed - and often empowered - by SCS however, one does not become - or some would say returns to be - the autonomous subject heralded by liberal humanism or transhumanism, but a technologically enfleshed self whose ontology is multiple and relational. If one's sense of self is disrupted or disintegrated by pain and pain medication and regained with SCS, in his becoming with SCS the recomposed bodyself is a singular plurality.

The body's materialisation with/in SCS and the recomposition of the (embodied) self is not necessarily lived and experienced as a positive, successful, satisfactory process of becoming. The process is different for every patient. Mr B for instance got SCS because of failed back surgery syndrome. He is very happy with his neuromodulation device because he can finally walk, use his car, take care of his partner who is in a wheelchair, go shopping or go to the sauna. He is especially satisfied with it because his tooth and knee implants are giving him a hard time. He is so content with it that he would like to have a zipper put in his back so that the world could see his implant. He is so pleased with it, that beeping every time he goes through security makes him laugh. 
In contrast, Mrs D is ambiguous about her device. Mrs D got SCS because of painful diabetic neuropathy. She does not like the device because she cannot see the screen of the remote control (she has poor eyesight because of diabetes) and the buttons are too small for her to use. Furthermore, she could not remember where the remote was before going to the hospital as she hid it in a drawer somewhere in the house. At the same time, she is happy with the device because, after the implantation, she was able to go shopping with her daughter for the first time. She abhors her device because it constant reminds her of diabetes. She loathes her device because she cannot touch her lower back without feeling the pulse generator. She finds it all the more abject as she hates the fact that her husband can also see it and feel it.

Mr B's and Mrs D's different and unique experiences with SCS draw attention to the fact that living with the neuromodulation device entails the materialisation of certain bodies and the enactment of a particular self. Both are situated, embodied and embedded, in different material-discursive relations and arrangements of technologies, bodies, kinship, histories, and locations. These arrangements are pivotal in one's positive and/or negative materialisation and composition with SCS. Living with the neuromodulation technology does not take place in a vacuum, but is a located process. As Donna Haraway reminds us, 'the body is always in-the-making; it is always a vital entanglement of heterogeneous scales, times, and kinds of beings webbed into fleshly presence, always a becoming, always constituted in relating' [Haraway 2008, p.163]. Living with SCS is indeed a vital entanglement.

\section{CONCLUSION}

Living with SCS is entering into an intimate relation with a(n implanted) technological device. Current frameworks within philosophy of technology are ill-suited for apprehending what is going on in this relationship. While postphenomenology, with its concept of technical mediation and its focus on human-technology relations, has drawn attention to the agency of (usable) technological artefacts [Ihde 1979, 1990; Verbeek 2005, 2006], it has however tended to neglect, and blackbox, the human in its material, corporeal reality. The works of Verbeek [2008, 2011] and Besmer [2012] have attempted to renew postphenomenological frameworks and enrich mediation theory to account for implanted technological devices. However, their emphasis on intentionality and conception of the body as a perceptual entity do not allow to completely comprehend what it means to live with SCS, as SCS is a 
somatechnology. It not only transforms but also enacts material bodies. One's kinaesthetic experience is transformed when SCS replaces the sensation of pain by paraesthesia. The body multiplies as it interacts with the technology: the body-in-paraesthesia materialises alongside the body-in-pain. Re-worlding takes place, which is empowering, however one's embeddedness in material-discursive relations can give rise to both distressing or satisfying compositions and lived experiences.

Attending to the experiences of patients living with SCS forces us to renew current philosophical frameworks. Bodies can no longer be silenced within philosophy of technology if we are to understand implanted technological artefacts. Furthermore, if these artefacts enter into close, intimate relationships with people, they also mobilise relationships and compositions beyond their material borders. The quality of the relationships between people and technology is crucial for the making and re-making of liveable compositions.

These considerations are not limited to the field of philosophy of technology alone. The practices of biomedical engineers, physicians and nurses might benefit as well from conceiving spinal cord stimulation as a somatechnology. Engineers might consider re-designing the remote control for it to meet the variety of individual needs: a smaller version for people who think it to be too bulky to use in public and a version with larger buttons and display for people with limited sight or sensory loss. Furthermore, during the development of new technologies, like paraesthesia-free stimulation paradigms, the effects on the incorporation processes and lives of the patients should be assessed. In current practices, the focus of the physicians and nurses is on optimizing stimulation settings. Yet, as incorporation processes are pivotal to live successfully with SCS, a physical therapist could be involved in the medical team in order to help patients experiment and familiarise with the new movements and postures that SCS effects. Finally, being implanted with a neuromodulation device means entering into diverse compositions. While the neurosurgeon currently focuses on the healing process, making sure that the implant is fixed, the role of the nurse and physician is cornerstone in counselling patients and setting the stimulation programmes. These are undoubtedly crucial aspects. However, for the medical team to facilitate the patient converting the medical experience with an implanted technology into a successful life with SCS, the team might benefit from consulting psychologists as well. It is important that the team engages with the patient and his or her relations and history, and acknowledges their material surroundings. 


\section{REFERENCES}

Baszanger I. Pain: Its Experience and Treatments. Social Science \& Medicine 1989; 29: 425-234

Besmer K. Embodying a Translation Technology: The Cochlear Implant and Cyborg Intentionality. Techné: Research in Philosophy and Technology 2002; 16: 296-316.

Breivik H, Collett B, Ventafridda V, Cohen R, Gallacher D. Survey of chronic pain in Europe: Prevalence, impact on daily life, and treatment. Eur J Pain 2006; 10: 287-333

Charmaz K. Stories of Suffering: Subjective Tales and Research Narratives. Qualitative Health Research 1999; 9: 362-382

De Preester H. Technology and the Body: The (Im)Possibilities of Reembodiment. Foundations of Science 2011; 16: 119-137

De Ridder D, Vanneste S, Plazier M, van der Loo E, Menovsky T. Burst Spinal Cord Stimulation: Toward ParesthesiaFree Pain Suppression. Neurosurgery 2010; 66: 986-990

De Ridder D, Plazier M, Kamerling N, Menovsky T, Vanneste S. Burst Spinal Cord Stimulation for Limb and Back Pain. World Neurosurg 2013. doi: 10.1016/j.wneu.2013.01.040

Haraway D. Simians, Cyborgs, and Women: The Reinvention of Nature. London: Free Association Books 1991

Haraway D. When Species Meet. Minneapolis: University of Minnesota Press 2008

IASP Task Force on Taxonomy, Part III: Pain Terms, A Current List with Definitions and Notes on Usage, in: Classification of chronic pain, ed. H. Merskey and N. Bogduk, $2^{\text {nd }}$ edition, IASP Press, Seattle, 1994, 209-214

Ihde D. Technics and Praxis: A Philosophy of Technology. Dordrecht: D. Reidel Publishing 1979

Inde D. Technology and the Lifeworld: From Garden to Earth. Bloomington and Minneapolis: Indiana University Press 1990

Ihde D. Ironic Technics. Copenhagen: Automatic Press 2008

Jackson JE. Chronic Pain and the Tension Between the Body as Subject and the Body as Object. In Embodiment and Experience: The Existential Ground of Culture and Self, edited by Thomas J. Csordas, 201-228. Cambridge: Cambridge University Press 1994

Lettow S. Somatechnologies: Rethinking the Body in the Philosophy of Technology." Techné: Research in Philosophy and Technology 2011; 15: 110-117

McGee EM. Bioelectronics and Implanted Devices. In Medical Enhancement and Posthumanity, edited by Bert Gordijn and Ruth Chadwick, 201-224. Springer 2008

Mol A. The Body Multiple: Ontology in Medical Practice. Durham and London: Duke University Press 2002

Nordmann A. If and Then: A Critique of Speculative NanoEthics." Nanoethics 2009; 1: 31-46

Sandberg A, Bostrom N. Converging Cognitive Enhancement." Annals of the New York Academy of Science 2006; 1093: 201-227

Scarry E. The Body in Pain. The Making and Unmaking of the World. New York and Oxford: Oxford University Press, 1985

Van Buyten JP, Al-Kaisy A, Smet I, Palmisani S, Smith T. High-Frequency Spinal Cord Stimulation for the Treatment of Chronic Back Pain Patients: Results of a Prospective Multicenter European Clinical Study. Neuromodulation 2012. doi: 10.1111/ner.12006

Vedder A, Klaming L. Human Enhancement for the Common Good, Using Neurotechnologies to Improve Eyewitness Memory. AJOB Neuroscience 2010; 1: 22-33

Verbeek PPCC. What Things Do: Philosophical Reflections on Technology, Agency, and Design. University Park: The Pennsylvania State University Press. 2005

Verbeek PPCC. Materializing Morality: Design Ethics and Technological Mediation. Science, Technology \& Human Values 2006; 31: 361-380

Verbeek PPCC. Cyborg Intentionality: Rethinking the Phenomenology of Human-Technology Relations. Phenomenology and the Cognitive Sciences 2008; 7: 387-395

Verbeek PPCC. Philosophy of Man and Technology. The Limits of Humanity: On Technology,Ethics, and Human Nature. University of Twente: Inaugural Lecture, 2009

Verbeek PPCC. Moralizing Technology: Understanding and Designing the Morality of Things. Chicago: The University of Chicago Press, 2011

Woopen C. Ethical aspects of neuromodulation. International review of neurobiology 2012; 107: 315-332 


\section{Chapter 10}

General discussion

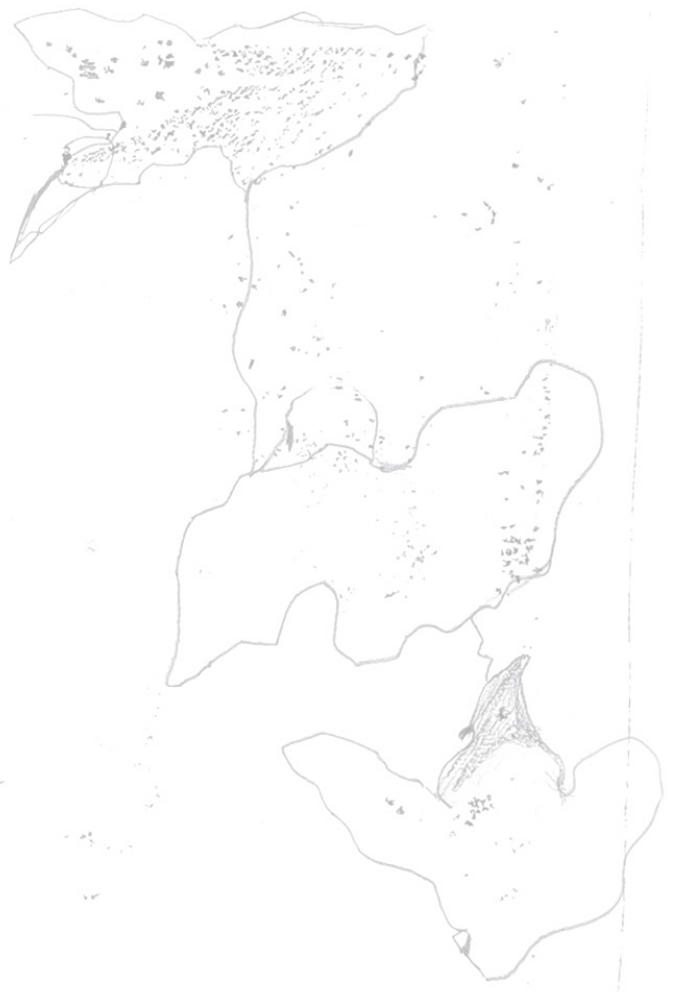


Chronic neuropathic pain interferes considerably with the quality of life and general functioning of many people. Despite the introduction of new drugs targeting neuropathic pain over the years, still only part of the patients obtains significant pain relief through medication. This motivates the search for alternative, non-pharmaceutical, therapies to target neuropathic pain. Spinal cord stimulation (SCS) is electrical stimulation of the dorsal columns of the spinal cord to interrupt or decrease neuropathic pain perception. This thesis reports on new clinical applications and technical developments of SCS for the treatment of neuropathic pain and reflects on what it actually means to live with an implanted neuromodulation device.

\section{CLINICAL STUDIES}

Chapter 2 and 3 report the results from our prospective pilot study on short-term and longterm effects of spinal cord stimulation (SCS) on pain intensity and quality of life in patients with painful diabetic neuropathy (PDN). Initially, eleven patients were included in the study; nine of them received a permanent SCS system and were followed. It was demonstrated that patients with PDN, who did not respond to conventional medical treatment, experienced significant pain reduction as well as reduction in medication intake and improvement of quality of life when they received SCS. Their average pain score was 77 at baseline and only 23 after twelve months of stimulation, while at that time six of the patients did not use any analgesic medication anymore. Despite the generally progressive nature of the disease, an initial positive response to SCS demonstrated to be stable up to at least six years, reflected in an average pain score of 27 for the remaining six patients. These very promising results suggested that SCS therapy should be considered in diabetic patients with neuropathic pain who do not respond to conventional treatment.

Limitations of the pilot study were the small number of patients who participated and the lack of a control group. To provide stronger evidence of the positive effects of SCS treatment for PDN we initiated and conducted an international multi centre randomised controlled trial, comparing the efficacy of SCS therapy to best conventional medical practice. The patients who participated in this trial are still followed, but the results from the first six months of the trial are already presented in Chapter 4. The average pain score at baseline was 73 for the SCS group and 67 for the control group. After six months of treatment the score was reduced to 29 for the SCS group and remained 66 for the control group. So, 
the randomised trial confirmed the results from the pilot study and demonstrated that compared with conventional pain treatment, SCS therapy causes significant pain reduction and improvement of quality of life in patients with refractory PDN in the lower extremities.

PDN is not a reimbursed indication for SCS in the Netherlands yet. Until now no large studies or randomised trials on SCS for PDN had been performed and the level of evidence was regarded not sufficient for reimbursement [Mailis-Gagnon 2004]. Considering the convincing results from both our pilot study and randomised trial, I believe PDN should become a reimbursed indication for SCS treatment. However, continued follow-up of a large group of patients with diabetic neuropathic pain and SCS is necessary to demonstrate the long-term effects of this treatment on the course of diabetic neuropathy and its associated pain.

Long-term follow up of 6 patients with insulin dependent diabetes mellitus (Chapter 3) suggests that SCS could reduce insulin needs, perhaps by reducing stress levels associated with chronic pain. If SCS improves the perfusion of the affected area, as suggested by several publications [e.g. Claeys 2000, Mannheimer 1998, Petrakis 2000], and the obtained pain reduction causes improved glycaemic control, SCS could slow down the progression of diabetic neuropathy. It would be interesting and advisable to perform a long-term cost effectiveness study on SCS in PDN.

Pain caused by diabetic neuropathy responds well to spinal cord stimulation. This might be due to the fact that PDN is a pure neuropathic pain without nociceptive components. Our randomised trial in 60 patients with PDN is the first randomised trial in patients with pure neuropathic pain. Given the positive results of our studies on PDN, we are starting pilot studies on the effects of SCS on other aetiologies of pure neuropathic pain, like neuropathic pain caused by radiotherapy or chemotherapy [Cata 2004], and expect comparable results.

\section{NEW TECHNOLOGICAL DEVELOPMENTS}

In addition to the evaluation of SCS treatment for PDN, we have also performed clinical evaluations of two new technological developments in SCS. Chapter 5 showed that recent emerged electrode leads, that combine features of percutaneous leads with features of surgical paddle leads, obtained comparable or better results in alleviating leg and low-back pain in failed back surgery syndrome (FBSS) than standard percutaneous leads. Forty-five 
patients participated in this observational study and at baseline the average score was 80 for leg pain and 75 for low back pain. After twelve months, the average scores were 32 for leg pain and 42 for low back pain. These scores are somewhat higher than the scores in patients with PDN, presumably caused by non-neuropathic pain components of FBSS that do not respond to SCS.

Besides, in this study in FBSS patients the hybrid leads were implanted via a surgical procedure, which is one of the major drawbacks of paddle leads [North 2002]. Pain and potential harm caused by this surgical procedure (unilateral interlaminar fenestration) will be diminished when a large diameter cannula or introducing device (Epiducer, St Jude Medical, Plano, TX, USA) is used to percutaneously implant these hybrid leads [Kinfe 2012]. However, these less invasive methods of placing the lead may hinder placement right onto the dura. Placing the lead via laminectomy allows direct visualization of placement and generally leads implanted with an open procedure are in closer contact with the dura, ensuring a better stimulation effect [Villavicencio 2000].

In Chapter 6 we evaluated burst spinal cord stimulation, a stimulation paradigm designed to induce pain reduction without eliciting the paraesthesia that accompanies conventional tonic SCS. This new stimulation paradigm was evaluated in 36 patients from Medisch Spectrum Twente who had previously participated in either the PDN trial (Chapter 4) or the study with percutaneous paddle leads (Chapter 5). Consequently, all these patients had experience with tonic SCS and the accompanying paraesthesias for at least six months. These patients represented a cross-section of the patients who receive SCS in Medisch Spectrum Twente. We demonstrated that about $60 \%$ of the patients with PDN or FBSS obtained further pain reduction with burst stimulation as compared to tonic stimulation. However, the patients with PDN who on average already responded better to SCS than the patients with FBSS benefitted relatively also most from switching to burst stimulation.

A third group was added to the study, consisting of 12 patients with low back and leg pain who did not participate in the study described in Chapter 5 and had reappearance of their pain despite tonic stimulation. This group of poor responders could not be treated with adjusting tonic stimulation anymore and would probably have their SCS system explanted. For each patient the cause of their reversion was unknown. The patients who had become poor responders to tonic stimulation benefitted least from burst stimulation, but still $50 \%$ of them experienced pain reduction with burst stimulation and preferred to keep the stimulator instead of having it explanted. 
Until now, burst stimulation could only be evaluated in clinical studies. By the end of 2013, burst stimulation will become available as a standard option in pulse generators manufactured by St Jude Medical. This will make it possible to program burst stimulation both in de novo SCS patients and in patients who already have a stimulator and test whether or not burst stimulation provides them with increased pain relief. This opportunity will presumably improve the general efficacy of SCS therapy.

It has been suggested [De Ridder 2010] that burst stimulation suppresses pain via the electro-physiological gate-control mechanism before the clinical paraesthesia threshold is reached, as the amplitude of effective burst stimulation pulses is lower than the amplitude of effective tonic stimulation pulses. However, the working mechanisms of burst stimulation and other high frequency stimulation paradigms [Van Buyten 2012, Tiede 2012] are still topic of debate and optimal stimulation settings will need to be studied both in models and in patients. So far, burst stimulation has been programmed as $40 \mathrm{~Hz}$ stimulation with burst consisting of five $500 \mathrm{~Hz}$ pulses, each with a duration of 1 ms. However, whether these settings provide optimal burst stimulation for all patients and for all indications has not been tested yet, but should be investigated in a large multi centre study.

Besides, the emerging of high frequency stimulation paradigms like burst stimulation, that are paraesthesie-free by nature, have cleared the way for randomised placebo-controlled trials [De Ridder 2013]. Until now, placebo-controlled studies had not been possible. With conventional tonic stimulation, the paraesthesia notifies the patient whether the stimulation is on or off, but with paraesthesia-free stimulation modes both blind and double-blind studies will be possible. However, when performing these studies it should be taken into account that burst stimulation will cost at least three times as much energy from the battery as tonic stimulation, while placebo will not cost any energy. Patients with rechargeable pulse generators will notice differences during recharge sessions.

Nevertheless, since the new stimulation paradigms with continuous or intermittent high frequency stimulation require much more energy from the battery than conventional tonic stimulation [De Ridder 2010], they are preferably programmed in rechargeable pulse generators. Programming anode-cathode stimulation configurations that require least energy will shorten the recharge duration or decrease the recharge frequency. The influence of the number of anodes and cathodes in stimulation configurations has been modelled (UT- 
SCS modelling software) and measured in patients. In Chapter 7 we reported that modelling might overestimate the effect compared to the clinical measurements, but that an increasing number of cathodes increased the energy consumption and that an increasing number of anodes decreased the energy consumption. The substantially smaller effects in energy consumption in the patients compared to the modelling experiments could be related to variations in the distance between the lead and spinal cord in the individual patients and to a smaller centre-to-centre distances between the active electrodes in patients compared to the modelled lead. The percutaneous paddle lead (Chapter 4, 5 and 6) has a centre-tocentre distance of $7 \mathrm{~mm}$, while this distance is $9 \mathrm{~mm}$ for the percutaneous lead used in the pilot study (Chapter 2 and 3) and for the UT-SCS model (Chapter 7). The superposition of two cathode fields and thus the decrease in threshold amplitude will be less when the cathodes are at a larger distance [Holsheimer 1998].

\section{CORTICAL MODULATIONS}

Chapter 8 describes our explorative study on pain evoked potentials measured in patients with SCS. We showed that we can record group based cortical alterations in pain processing between patients who respond favourably to burst spinal cord stimulation and patients who do not. The magnitude of pain relief obtained by burst spinal cord stimulation seems to be correlated with the reduction of the contralateral P200 amplitude of the pain evoked potential. The better the pain reduction in a patient, the more the pain evoked potential resembled the evoked potential of healthy, pain-free control subjects. However, a larger group of patients with a greater variety in responses to spinal cord stimulation needs to be studied, preferably combined with imaging and source localisation techniques.

Imaging patients with a spinal cord stimulator with MRI or fMRI of has only become possible since MRI-compatible SCS-systems have become available [Moens 2012a; Moens 2012b]. This offers exciting new opportunities. Studies combining functional responses with images of the nervous system may provide more details about the localisation and extent of the presumed cortical networks involved in pain processing in patients with chronic pain, and could reveal modulations of this network due to successful or unsuccessful SCS treatment. 


\section{LIVING WITH SCS}

In Chapter 9 we have explored implications of living with a spinal cord stimulator from a patient perspective and related them to philosophical frameworks for apprehending humantechnology relations. Based on interviews with patients living with SCS we concluded that SCS requires active incorporation and composing processes. However, current philosophical approaches are ill-suited for comprehending the implications of the incorporation processes and what it means to live with SCS. We propose to renew philosophical frameworks and take into account how bodies are transformed and enacted when patients enter an intimate relation with an implanted technological device like a spinal cord stimulator. Consequences of these reflections are certainly not limited to philosophical frameworks only. Professionals, like physicians and biomedical engineers, will also benefit from insight in the incorporation processes when they are counselling patients or designing new technology.

\section{CONCLUSIONS}

In this thesis, clinical as well as technological and philosophical aspects of spinal cord stimulation for the treatment of neuropathic pain have been reported and discussed. We have shown that spinal cord stimulation causes significant pain reduction and improvement of quality of life in patients with painful diabetic neuropathy. We have also shown that new technological developments, like new lead designs and new stimulation paradigms, will probably further increase the efficacy of SCS therapy. Finally, we showed that living successfully with a spinal cord stimulator requires patients to enter an intimate relationship with the device. Insight in the incorporation processes when counselling patients or when designing new technology is a prerequisite for further improving spinal cord stimulation therapy. Because of the intricate character of both pain and spinal cord stimulation, I believe that multidimensional approaches are most promising for extending our knowledge on all aspects of pain and for finding new treatment options 


\section{REFERENCES}

Cata JP, Cordella JV, Burton AW, Hassenbusch SJ, Weng HR, Dougherty PM. Spinal Cord Stimulation relieves chemotherapy-induced pain: a clinical case report. J Pain Symptom Manage 2004;27:72-78

Claeys LGY. Effects of Spinal Cord Stimulation on Nutritional Skin Blood Flow in Patients with Ischemic Pain. Neuromodulation 2000;3:123-130

de Ridder D, Vanneste S, Plazier M, van der Loo E, Menovsky T. Burst Spinal Cord Stimulation: Toward ParesthesiaFree Pain Suppression. Neurosurgery 2010;66:986-990

de Ridder D, Plazier M, Kamerling N, Menovsky T, Vanneste S. Burst Spinal Cord Stimulation for Limb and Back Pain. World Neurosurg 2013. doi: 10.1016/j.wneu.2013.01.040

Holsheimer J. Computer modelling of spinal cord stimulation and its contribution to therapeutic efficacy (review). Spinal Cord 1998;36:531-540

Kinfe TM, Schu S, Quack FJ, Wille C, Vesper J. Percutaneous Implanted Paddle Lead for Spinal Cord Stimulation: Technical Considerations and Long-Term Follow-Up. Neuromodulation 2012; 15: 402-407

Mailis-Gagnon A, Furlan, MD PhD AD, Sandoval JA, Taylor RS. Spinal cord stimulation for chronic pain. Cochrane Database of Systematic Reviews 2004, Issue 3. Art. No.: CD003783. DOI: 10.1002/14651858.CD003783. pub2.Mannheimer 1998

Mannheimer C, Eliasson T, Augustinsson LE et al. Electrical stimulation versus coronary artery bypass surgery in severe angina pectoris: the ESBY study. Circulation 1998;97(12):1157-1163.

Moens M, Droogmans S, Spapen H, de Smedt A, Brouns R, van Schuerbeek P, Luypaert R, Poelaert J, Nuttin B. Feasibility of cerebral magnetic resonance imaging in patients with externalised spinal cord stimulator. Clinical Neurology and Neurosurgery 2012;114: 135- 141

Moens M. Sunaert S, Mariën P, Brouns R, de Smedt S, Droogmans S, van Schuerbeek P, Peeters R, Poelaert J, Nuttin B. Spinal cord stimulation modulates cerebral function: an fMRI study. Neuroradiology 2012; 54:1399-1407

Petrakis, I. E., \& Sciacca, V. (2000). Epidural spinal cord electrical stimulation in diabetic critical lower limb ischemia. Journal of diabetes and its complications, 13:293-9.

Tiede J, Brown L, Gekht G, Vallejo R, Yearwood T, Morgan D. Novel spinal cord stimulation parameters in patients with predominant back pain. Neuromodulation 2012. doi:10.1111/ner.12032

van Buyten JP, Al-Kaisy A, Smet I, Palmisani S, Smith T. High-Frequency Spinal Cord Stimulation for the Treatment of Chronic Back Pain Patients: Results of a Prospective Multicenter European Clinical Study. Neuromodulation 2012. doi: 10.1111/ner.12006

Villavicencio AT, Leveque JC, Rubin L, Bulsara K, Gorecki JP. Laminectomy versus percutaneous electrode placement for spinal cord stimulation. Neurosurgery 2000;46:399-405 



\section{Summary}

Samenvatting

Dankwoord

\section{Curriculum Vitae}

\section{Publications}




\section{SUMMARY}

This thesis reports on the opportunities of several new applications of spinal cord stimulation for the treatment of neuropathic pain. Clinical as well as technological and philosophical aspects are described and discussed.

Chapter 2 and 3 report the results from our pilot study on short-term and long-term effects of spinal cord stimulation (SCS) in patients with painful diabetic neuropathy (PDN). It was demonstrated that patients, who did not respond to conventional medical treatment, experienced significant pain reduction as well as reduction in medication intake and improvement of quality of life when they received SCS. Chapter 4 presents the results from the consecutively performed international multi centre randomised clinical trial. We show that SCS offers an effective therapy for chronic PDN. SCS causes significant pain reduction and improvement of quality of life. Despite the progressive nature of diabetic neuropathy, the pain relief shows to be sustainable over the long term.

Chapter 5 reports on the performance of a new percutaneous paddle lead in patients with failed back surgery syndrome (FBSS). The new electrode lead that is paddle shaped but can be implanted percutaneously, is safe and causes significant pain relief in both the low back and the lower limbs. The results are comparable or compare favourably to earlier studies of FBSS patients treated with SCS. Chapter 6 presents the results of an evaluation of the recently emerged burst stimulation paradigm, that combines features of high frequency stimulation with conventional, tonic stimulation. About $60 \%$ of the 48 patients with either PDN or FBSS who already had tonic SCS for over six months experienced further pain reduction upon application of burst stimulation.

Chapter 7 shows that when the paraesthesia area can be covered with several electrode contact configurations, it will be beneficial for the patient to program a configuration with one cathode and either no or multiple anodes, as these configurations will cost least energy.

Chapter 8 describes our explorative study on pain evoked potentials measured in patients with SCS. We showed that we can record group based cortical alterations in pain processing between patients who respond favourably to burst SCS and patients who do not. The magnitude of pain relief obtained by burst SCS seems to be correlated with the reduction of the contralateral P200 amplitude of the pain evoked potential. 
Finally, in Chapter 9 we have explored implications of living with a spinal cord stimulator from a patient perspective and related them to philosophical frameworks for apprehending human-technology relations. We showed that living successfully with a spinal cord stimulator requires patients to enter an intimate relationship with the device. Insight in these incorporation processes when counselling patients or when designing new technology is a prerequisite for improving spinal cord stimulation therapy. 


\section{SAMENVATTING}

In dit proefschrift wordt een aantal studies beschreven op het gebied van ruggenmergstimulatie (SCS) voor de behandeling van neuropathische pijn. Nieuwe klinische en technologische ontwikkelingen worden geëvalueerd en tot slot worden filosofische aspecten besproken.

In hoofdstukken 2 en 3 worden eerst de korte en lange termijn effecten van SCS voor de behandeling van diabetische neuropathische pijn (DNP) gerapporteerd. Deze pilot studie laat zien dat patiënten met DNP die geen of onvoldoende baat hebben bij pijnbestrijding met medicijnen, met SCS wel significante pijnvermindering ervaren. Aansluitend op de pilot studie is een internationale, gerandomiseerde studie uitgevoerd (hoofdstuk 4). In deze studie hebben we laten zien dat SCS een effectieve behandeling is voor DNP. Naast pijnvermindering zorgt SCS ook voor verbetering van kwaliteit van leven. Deze positieve effecten blijken ook op de lange termijn nog aanwezig te zijn.

In hoofdstuk 5 zijn de mogelijkheden onderzocht van een nieuwe, smalle plaatelektrode die via een minimaal invasieve methode geïmplanteerd kan worden. Deze elektrode is gebruikt voor SCS bij mensen met lage rug pijn (FBSS) en de studie laat zien dat de elektrode veilig is en voor significante pijnreductie zorgt in zowel de rug als de benen.

Zowel patiënten met DNP (hoofdstuk 4) als met FBSS (hoofdstuk 5) hebben twee weken lang burst-stimulatie uitgeprobeerd, een nieuwe stimulatievorm waarbij puls-treintjes van 500 $\mathrm{Hz}$ met een frequentie van $40 \mathrm{~Hz}$ aan het ruggenmerg worden afgegeven. Hoofdstuk 6 laat zien dat ongeveer $60 \%$ van deze patiënten nog weer extra pijnvermindering heeft als gevolg van burst-stimulatie, in het algemeen zonder dat de stimulatie gevoeld wordt.

Wanneer eenzelfde mate van pijnvermindering bereikt kan worden met verschillende combinaties van actieve elektrodes, is het energetisch het gunstigst om te kiezen voor een combinatie met één kathode en geen of juist een groot aantal anodes. In hoofdstuk 7 wordt aangetoond dat dit niet alleen uit computermodellen blijkt, maar ook bij patiëntmetingen het geval is. 
De studie beschreven in hoofdstuk 8 laat zien dat het mogelijk is om met EEG sensorisch evoked potentials te meten bij patiënten met SCS en dat de corticale verwerking van de stimuli mogelijk anders verloopt in patiënten die baat hebben bij burst-stimulatie dan bij patiënten die er geen baat bij hebben. De mate van pijnvermindering lijkt gecorreleerd met de afname in de contralaterale P200 piek van de evoked potential.

Tenslotte hebben we onderzocht wat voor de patiënt de gevolgen zijn van het moeten leven met SCS en hebben we de verhalen van patiënten vergeleken met hoe er binnen de filosofie naar mens-techniek relaties gekeken wordt. In hoofdstuk 9 laten we zien dat patiënten een intieme relatie met de ruggenmergstimulator aangaan wanneer de technologie een onderdeel van hun lichaam wordt. Inzicht hebben in de manieren waarop deze relaties vorm krijgen en wat de consequenties er van zijn, is noodzakelijk om zowel de behandeling met SCS als de ontwikkeling van nieuwe technologie verder te kunnen optimaliseren. 


\section{DANKWOORD}

Gedurende de afgelopen jaren heb ik met heel veel mensen samengewerkt. Ik hoop dat ik er in de afgelopen jaren geen misverstand over heb laten bestaan en dat iedereen allang weet hoe zeer ik alle samenwerking waardeer. Toch wil ik op deze plek graag iedereen nogmaals bedanken voor de hulp bij het uitvoeren van het onderzoek en het tot stand komen van dit proefschrift. Een aantal mensen wil ik hier met name noemen.

Niets van het werk beschreven in dit proefschrift had gerealiseerd kunnen worden zonder de medewerking van de patiënten met een ruggenmergstimulator. In de eerste plaats wil ik dan ook graag alle patiënten hartelijk bedanken voor hun deelname aan de studies. Waarbij ik in het bijzonder de mensen wil bedanken op wie ik herhaaldelijk een beroep mocht doen.

Dan Mathieu, zonder jou had ik al dit onderzoek nooit kunnen uitvoeren. Bedankt voor de mogelijkheden die je me biedt, de plezierige samenwerking, het vertrouwen, de voortdurende steun en je relativerende kijk op zaken.

Michel, bedankt dat je mijn promotor wilt zijn en me de gelegenheid biedt om binnen de vakgroep $\mathrm{CNPH}$ te promoveren. Daarnaast wil ik ook de andere leden van de promotiecommissie, Jan Holsheimer, Frank Huygen, Kaare Meier, Hans Rietman, Peter Veltink en Peter-Paul Verbeek, bedanken voor hun bijdragen aan mijn promotie.

Jessica, je onderschat het belang van een fijne kamergenoot, bedankt voor je betrokkenheid en je geduld.

Alle onderzoekers die meegewerkt hebben aan de onderzoeken die onderdeel zijn van dit proefschrift, wil ik bedanken voor de prettige samenwerking en hun bijdragen aan het werk. Een paar mensen wil ik in het bijzonder bedanken: Rik Buschman, omdat hij mij in het neuromodulatie onderzoek geïntroduceerd heeft. De onderzoekers van de ziekenhuizen in Aarhus, Harderwijk, Nieuwegein, Hasselt, Düsseldorf en Odense voor hun inspanningen om van de diabetische neuropathie studie een succes te maken. Marjanne voor haar inzet bij de burst studies. Lucie voor de interessante discussies. En Klaas voor de hulp bij het ontcijferen en invoeren van data. 
Mijn collega's van het Medisch Spectrum Twente wil ik bedanken voor hulp en (morele) ondersteuning: Cindy, Rian, neurochirurgen, secretaresses van poli 30, KNF laboranten, secretaresses van poli 13, neurologen, klinisch fysici en klinisch fysici in opleiding, medische techniek, psychologen, anesthesiologen en Job.

De aio's (Bas-Jan, Chin, Esther, Floor, Marleen, Shaun) en oud-aio's (Esther, Hanneke, Meyke, Sid) van onder andere de vakgroep CNPH, mijn hartelijke dank voor jullie steun, de hulp bij metingen, de gezamenlijke congresbezoeken en de gezelligheid in het algemeen.

Medewerkers en voormalige medewerkers van ANS, later SJM, wil ik bedanken voor het mogelijk maken van de diabetische neuropathie studie, met name Andre Elands, Koert Hermans, Wim Polet en Hindrik Robbe.

Susan, Patricia, Ria, Benjamin, Daphne, Jelmer, Arjan, Mariella, Lili-Marjan, Daan, Michelle, Marleen, Leonie, Lonneke, Maryse, Yoel, Niek, Janita, Mike, Matthias, Frank, Hans, Daphne, Erik, Marleen, Rob, Juliet, Marjolijn, Marjanne, Inger, Kim, Linda, Lieke, Anniek, Patriek, verschillende MDO-groepjes en PWS-groepjes, jullie heb ik de afgelopen jaren mogen begeleiden. Lang niet van iedereen is het werk terug te vinden in dit proefschrift, maar ik wil jullie allemaal bedanken voor de samenwerking en voor wat ik van jullie heb geleerd.

Alle vrienden en Corry, Schil en Schilleman, bedankt dat jullie er voor me zijn wanneer dat nodig is. 


\section{CURRICULUM VITAE}

Cecile de Vos obtained her MSc degree in Applied Physics from the University of Twente in 2002 and started as a trainee medical physicist in Medisch Spectrum Twente hospital before she was able to finish her MA in Philosophy of Science, Technology and Society. Her fouryear training as a medical physicist included a wide variety of projects within almost every department of the hospital and she has been a medical physicist at the departments of Neurosurgery and Clinical Neurophysiology since its completion. She completed an additional MSc in Neuroscience at the Erasmus University Rotterdam in 2009. Various research projects on spinal cord stimulation for the treatment of neuropathic pain performed since 2007 have resulted in this PhD thesis. 


\section{PUBLICATIONS}

CC de Vos, SM van Maarseveen, PJAM Brouwers, MJAM van Putten. Continuous EEG monitoring during thrombolysis in acute hemispheric stroke patients using the brain symmetry index. Journal of Clinical Neurophysiology 25(2), p.77-82, 2008

CC de Vos, V Rajan, W Steenbergen, HE van der Aa, HPJ Buschman. Effect and safety of spinal cord stimulation for treatment of chronic pain caused by diabetic neuropathy. Journal of Diabetes and its Complications 23(1), p.40-45, 2009

CC de Vos, MP Hilgerink, HPJ Buschman, J Holsheimer. Electrode contact configuration and energy consumption in spinal cord stimulation. Neurosurgery 65(6), p.ons210-217, 2009

CC de Vos, L Melching, J van Schoonhoven, JJ Ardesch, AW de Weerd, HCE van Lambalgen, MJAM van Putten. Predicting success of vagus nerve stimulation (VNS) from interictal EEG. Seizure; European Journal of Epilepsy 20(7), p.541-545, 2011

MC Cloostermans, CC de Vos, MJAM van Putten. A novel approach for computer assisted EEG monitoring in the adult ICU. Clinical Neurophysiology 122(10), p.2100-2109, 2011

CC de Vos, C Dijkstra, MWPM Lenders, J Holsheimer. Spinal cord stimulation with hybrid lead relieves low-back and leg pain. Neuromodulation 15(2), p.118-123, 2012

ML Rots, CC de Vos, JS Smeets-Schouten, R Portier, MJAM van Putten. Suppressors of interictal discharges in idiopathic childhood occipital epilepsy of Gastaut. Epilepsy \& Behavior 25, p.189-191, 2012

T Heida, N Poppe, CC de Vos, MJAM van Putten, J van Vught.

The mirror neuron system in Parkinson's disease. (submitted)

CC de Vos, MJ Bom, S Vanneste, MWPM Lenders, D de Ridder.

Burst spinal cord stimulation evaluated in patients with failed back surgery syndrome and painful diabetic neuropathy. (submitted) 
EM ter Braack, CC de Vos, MJAM van Putten.

Masking the auditory evoked potential in TMS-EEG: a comparison of various methods. (submitted)

CC de Vos, MWPM Lenders, MJAM van Putten.

Long term effects of spinal cord stimulation in patients with painful diabetic neuropathy. (submitted)

L Dalibert, CC de Vos.

Living and composing with a somatechnology. (submitted)

ES te Woerd, CC de Vos, T Heida, MJAM van Putten.

Quantifying tinnitus with auditory evoked potentials. (submitted) 
